U. S. DEPARTMENT OF THE INTERIOR

GEOLOGICAL SURVEY

\title{
Gold and spectrographic analyses of 110 outcrop and 238 B-horizon soil samples from the western vermilion district, northeastern Minnesota
}

\author{
By
}

J.B. McHugh, * R.T. Hopkins, * H.V. Alminas, * and G.T. Hill*

$$
\begin{aligned}
& \text { Open-File Report } 92-615 \text { A Paper Version } \\
& 92-615 \text { B Diskette Version }
\end{aligned}
$$

This report is preliminary and has not been reviewed for conformity with U.S. Geological Survey editorial standards or with the North American Stratigraphic Code. Any use of trade, product or firm names is for descriptive purposes only and does not imply endorsement by the U.S. Government.

*U.S. Geological Survey, DFC, Box 25046, MS 973, Denver, CO 80225 


\section{CONTENTS}

Introduction............................. 1

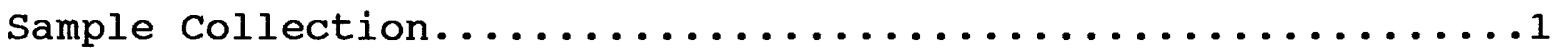

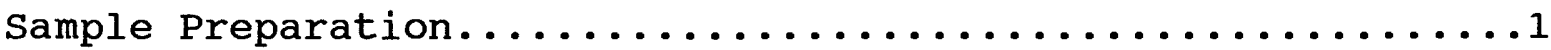

Analytical Procedures......................... 2

References Cited............................4

\section{ILLUSTRATIONS}

Figure 1. Index map of the study area.............. 3

\section{TABLES}

Table 1. Gold and spectrographic analyses of 110 outcrop samples from the west Vermilion district, northeastern Minnesota............................. 5

Table 2. Gold and spectrographic analyses of 238 B-horizon soil samples from the west Vermilion district, northeastern Minnesota......................... 17 


\section{INTRODOCTION}

The study area within the west Vermilion district, as referred to in this report, encompasses some $428 \mathrm{~km}^{2}$ (Fig. 1). It is a westward extension of an area described in U.S. Geological Survey Bulletin 1984 (Alminas and others, 1992). The silver and base-metal data for the eastern study area is listed in U.S. Geological Survey Open-File Report 81-999 (Grimes and others, 1981) and the gold data is listed in U.S. Geological Survey Open-File 90-86 (McHugh and others, 1990).

The greenstone belt is composed of mafic metavolcanic and associated rocks that are intruded on both the north and south by major granitoid bodies. These rocks have mineral assemblages characteristic of the greenschist-facies metamorphism. The general geology of the district has been described by Sims (1976). Pleistocene glacial materials consisting of till, outwash and lacustrine deposits, associated with the wisconsin episode of glaciation, cover the area. These are the parent materials of the B-horizon soils sampled here.

The field work was completed in June of 1991. A digital version of this data report is available in U.S. Geological Survey Open-File Report 92-615 B.

\section{SAMPLE COLLECTION}

B-horizon soil samples were collected at 238 localities along roads, rivers and lake shores. Outcrop samples were collected at 110 of these sites (within $6.7 \mathrm{~m}$ of the soil site) and have the same latitudinal and longitudinal parameters as the corresponding soils. The B-horizon soil samples were collected at a depth of 30 to $45 \mathrm{~cm}$. Although variable from site to site, these soils are generally fine to medium grained with a low to moderate organic content and range in color from yellow through red to light brown. Characteristically the B-horizon soils contain higher concentrations of $\mathrm{Fe}$ and $\mathrm{Mn}$ oxides than $\mathrm{A}$-horizon soils, have a substantially lower organic content, and are coarser with a greater content of fragmental rock material.

Outcrop samples were collected as composited chip samples and generally incorporated substantial weathered surface material.

\section{SAMPLE PREPARATION}

The soil samples were air-dried in the original cloth sample bag. Extremely clay-rich samples were disaggregated in a jaw crusher, using a wide jaw setting. All of the soils were then

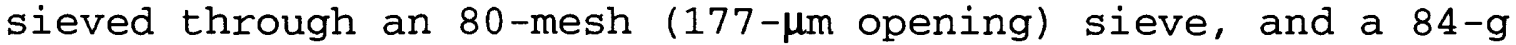
(3-oz) container of the fine fraction was saved for analysis. Outcrop samples were crushed in a jaw crusher and ground in a vertical grinder to approximately $105 \mu \mathrm{m}$. 


\section{ANALYTICAL METHODS}

Each sample was analyzed for 35 elements using a semiquantitative direct-current arc emission spectrographic method (Grimes and Marranzino, 1968). Spectrographic results were obtained by visual comparison of spectra derived from the sample against spectra obtained from standards made from pure oxides and carbonates. Standard concentrations are geometrically spaced over any given order of magnitude of concentrations as follows: $100,50,20,10$ and so forth. Samples whose concentrations are estimated to fall between those values are assigned values of $70,30,15$, and so forth. The precision of the analytical method has been determined to be within one reporting interval 83 percent of the time and within two reporting intervals 96 percent of the time (Motooka and Grimes, 1976).

The gold content of the outcrop and soil samples was determined using an atomic-absorption spectrophotometric method described below:

A 5-gm sample is roasted for 1 hour at $680^{\circ} \mathrm{C}$. Gold is then extracted with a hydrobromic acid- 0.5 bromine solution and MIBK (methyl isobutyl ketone). Electrothermal atomic-absorption spectroscopy, using background correction, is used to determine gold to $0.001 \mathrm{ppm}$ (1 ppb) (O'Leary and Meier, 1986). 
U.S. Geological Survey Topographic Maps:

1. Haley 7.5'

2. Norwegian Bay 7.5'

3. Vermilion Dam 7.5'

4. Cook 7.5'

5. Sassas Creek $7.5^{\prime}$

6. Lost Lake 7.5'

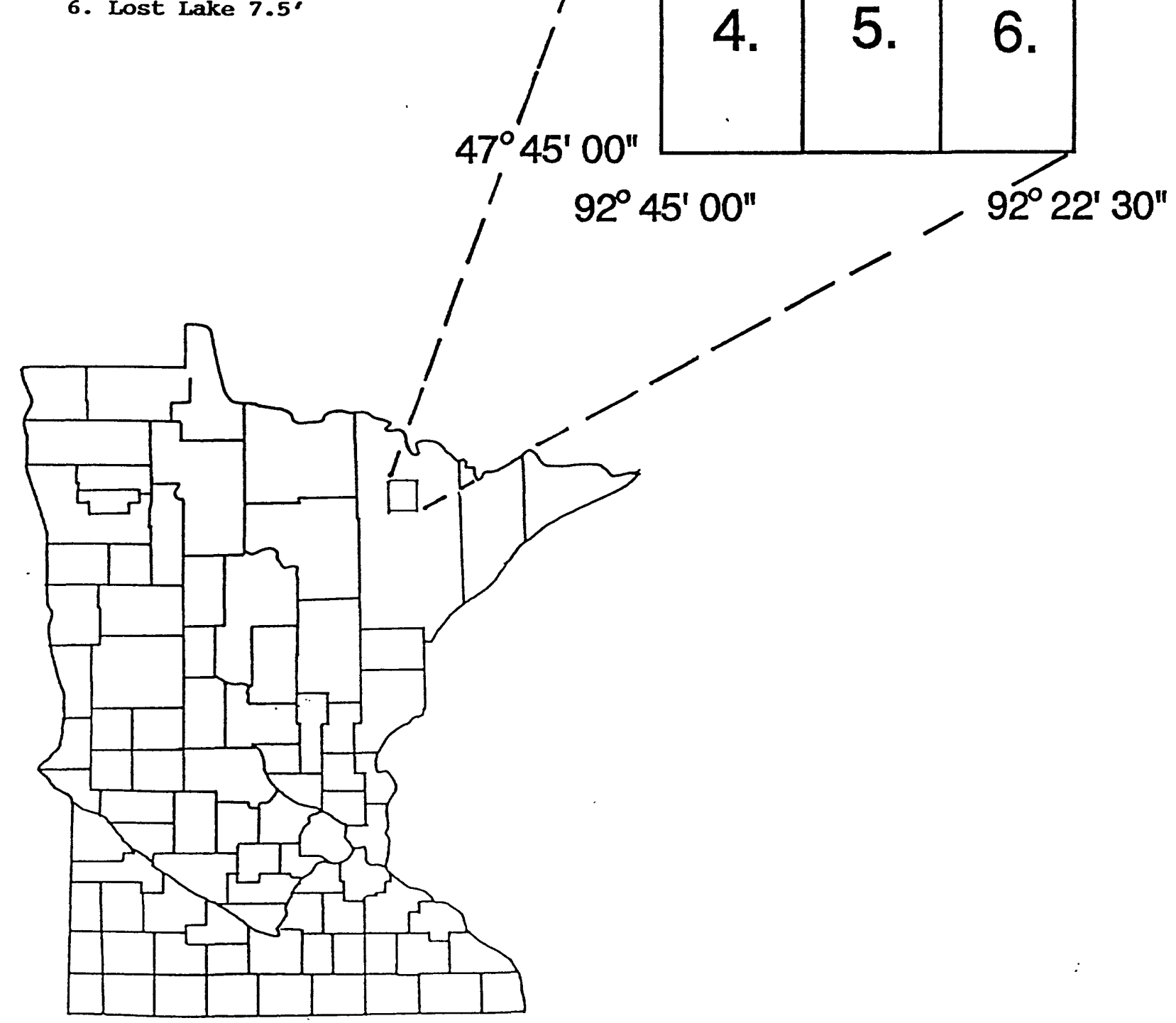

Figure 1. Index map of the study area in the west

Vermilion district, northeastern Minnesota. 


\section{REFERENCES CITED}

Alminas, H.V., McHugh, J.B., and Perry, E.C.Jr., 1992, Preciousand Base-Metal Mineralization in the West-Central Vermilion District, Portions of St.Louis, Lake, and Cook Counties, Northeastern Minnesota: U.S. Geological Survey Bulletin $198437 \mathrm{p}$.

Grimes, D.J., and Alminas, H.V., 1981, Analyses of A-horizon soil and outcrop samples from the Vermilion district, northeastern Minnesota: U.S. Geological Survey Open-File Report 81-99936 p.

Grimes, D.J., and Marranzino, A.P., 1968, Direct-current arc and alternating-current arc spark emission spectrographic field methods for the semiquantitative analysis of geologic materials: U.S. Geological Survey Circular 591, 6 p.

McHugh, J.B., Alminas, H.V., and Perry, E.C.Jr., 1990, Gold contents of 766 A-horizon soil samples from the Vermilion District, northeastern Minnesota: U.S. Geological Survey Open-File Report 90-86, 20 p.

Motooka, J.M., and Grimes, D.J., 1976, Analytical precision of one-sixth order semiquantitative spectrographic analyses: U.S. Geological Survey Circular 738, 25 p.

O'Leary, R.M., and Meier, A.L., 1986, Analytical Methods used in Geochemical E.exploration, 1984: U.S. Geological Survey Circular $948,48 \mathrm{p}$.

Sims, P.K., 1976, Early Precambrian tectonic-igneous evolution in the Vermilion district, northeastern Minnesota: Geological Society of America Bulletin v. 87, p.379-389. 
Table 1. Gold and spectrographic analyses of 110 outcrop samples.

[N, not detected; <, detected but below the limit of determination shown; >, determined to be greater than the value shown.]

\begin{tabular}{|c|c|c|c|c|c|c|c|c|c|}
\hline Sample & Latitude & Longitude & $\mathrm{Ca}: \mathrm{o}-\mathrm{s}$ & $\because-s$ & $\because-5$ & $\mathrm{Na} \quad:-\mathrm{s}$ & $\because-s$ & $\mathrm{Ti} \quad \because-\mathrm{s}$ & Ag ppm-s \\
\hline WV0002R & 475456 & 921950 & .3 & .5 & .1 & 2 & $<.2$ & .07 & $<.5$ \\
\hline WV0004R & $4754 \quad 40$ & $92 \quad 22 \quad 2$ & .1 & .5 & .2 & 2 & $<.2$ & .03 & $\mathrm{~N}$ \\
\hline WV0005R & $4754 \quad 13$ & 922258 & .3 & 3 & 1 & 1.5 & $<.2$ & .3 & $\mathrm{~N}$ \\
\hline WV0006R & 475354 & $92 \quad 23 \quad 1$ & 5 & 5 & 1.5 & 2 & $N$ & 1 & N \\
\hline WV0007R & 475358 & 922323 & 1 & 5 & 1.5 & 2 & $<.2$ & .5 & N \\
\hline WV0009R & 475428 & $92 \quad 2725$ & .5 & 5 & 1.5 & 2 & $<.2$ & .5 & N \\
\hline WV0011R & $4754 \quad 5$ & $92 \quad 2648$ & .7 & 3 & 1 & 2 & $<.2$ & .3 & N \\
\hline WV0012R1 & $4754 \quad 34$ & $92 \quad 2552$ & 7 & 3 & 2 & 1.5 & $\mathrm{~N}$ & .7 & $\mathrm{~N}$ \\
\hline WN0012R2 & 475434 & 922552 & .3 & .5 & .3 & 2 & $<.2$ & .07 & N \\
\hline WV0013R & 475417 & 922536 & .7 & 2 & 1 & 5 & $<.2$ & .3 & $N$ \\
\hline WV0014R & 475422 & 92256 & .3 & 1.5 & .15 & 2 & $<.2$ & .02 & $<.5$ \\
\hline WV0016R & 475331 & 922630 & .7 & 5 & 1.5 & 1.5 & $<.2$ & .5 & .7 \\
\hline WV0017R & $4753 \quad 46$ & $92 \quad 2552$ & .3 & 5 & 3 & 3 & $<.2$ & .3 & $<.5$ \\
\hline WV0018R & 475353 & $92 \quad 2518$ & .7 & 5 & 2 & 2 & $<.2$ & .3 & $N$ \\
\hline WV0019R & $4754 \quad 6$ & $9225 \quad 5$ & 3 & 5 & 2 & 2 & $<.2$ & .3 & $\mathrm{~N}$ \\
\hline WV0021R & $4754 \quad 5$ & $92 \quad 24 \quad 19$ & 1.5 & 5 & 1.5 & 3 & $<.2$ & .5 & .5 \\
\hline WV0022R & $47 \quad 54 \quad 12$ & 922350 & 3 & 5 & 3 & 3 & $N$ & .5 & $N$ \\
\hline WV0023R & $4754 \quad 27$ & $92 \quad 2325$ & 1 & .7 & .3 & 2 & $<.2$ & .07 & $N$ \\
\hline WV0024R & $4754 \quad 30$ & $92 \quad 2250$ & .5 & 1.5 & .7 & 3 & $<.2$ & .2 & $\mathrm{~N}$ \\
\hline WV0026R & 475326 & $9224 \quad 9$ & 10 & 5 & 3 & 1.5 & $\mathrm{~N}$ & .7 & N \\
\hline WV0027R & $4753 \quad 15$ & $92 \quad 24 \quad 22$ & $<.05$ & .1 & .02 & .2 & $<.2$ & .003 & $\mathrm{~N}$ \\
\hline WV0029R & 474738 & $92 \quad 23 \quad 42$ & .3 & 2 & 1 & 1.5 & $<.2$ & .3 & N \\
\hline WV0030R & $47 \quad 48 \quad 10$ & $92 \quad 2338$ & 1.5 & 3 & 1 & 2 & $<.2$ & .2 & $<.5$ \\
\hline WV0030R & $4748 \quad 10$ & $92 \quad 23 \quad 38$ & .7 & .7 & .3 & 2 & $N$ & .07 & $\mathrm{~N}$ \\
\hline WV0032R & $47 \quad 4859$ & $92 \quad 24 \quad 12$ & .7 & 1 & .2 & 1.5 & N & .15 & $<.5$ \\
\hline WV0042R & $\begin{array}{lll}47 & 48 & 1\end{array}$ & 922729 & .1 & 1 & .3 & 5 & $\mathrm{~N}$ & .15 & $N$ \\
\hline WV0043R & 474655 & $92 \quad 26 \quad 43$ & .5 & 2 & 1 & 2 & $<.2$ & .5 & $N$ \\
\hline WV0052R & $4746 \quad 40$ & 922758 & 3 & 3 & 2 & 3 & $\mathbf{N}$ & .5 & N \\
\hline WV0055R & $47 \quad 46 \quad 8$ & $9230 \quad 1$ & .15 & .7 & .3 & 2 & 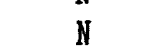 & .15 & N \\
\hline WV0056R & 474635 & $9230 \quad 3$ & 5 & 3 & 1.5 & 3 & .3 & .7 & N \\
\hline WW0068R & $47 \quad 46 \quad 27$ & 923145 & 1 & 3 & 1.5 & 2 & $<.2$ & .3 & N \\
\hline WV0071R & 474738 & 923138 & .7 & 1 & .7 & 2 & $<.2$ & .1 & $\mathrm{~N}$ \\
\hline WV0071R1 & 474738 & 923138 & .7 & 2 & .5 & 2 & .2 & .7 & $<.5$ \\
\hline WV0079R & $\begin{array}{lll}47 \quad 45 & 42\end{array}$ & 923359 & 5 & 3 & 2 & 3 & $N$ & .5 & N \\
\hline WV0112R & $4752 \quad 1$ & $92 \quad 25 \quad 12$ & 1 & 3 & 1.5 & 3 & N & .5 & N \\
\hline WV0113R & $4752 \quad 1$ & 922433 & 3 & 3 & 2 & 2 & $<.2$ & .5 & N \\
\hline WV0114R & $4752 \quad 4$ & $9224 \quad 1$ & 7 & 5 & 3 & 3 & $<.2$ & .5 & N \\
\hline WV0116R & $4752 \quad 19$ & $92 \quad 22 \quad 44$ & 5 & 5 & 1.5 & 1 & N & .5 & $N$ \\
\hline WV0117R & $4752 \quad 20$ & $92 \quad 21 \quad 15$ & 1 & 3 & 1.5 & 2 & $<.2$ & .5 & 1 \\
\hline WV0118R & $4752 \quad 26$ & $92 \quad 2218$ & 5 & 5 & 1.5 & 1 & $\mathrm{~N}$ & .7 & N \\
\hline WV0119R & $47 \quad 52 \quad 45$ & 922238 & .3 & 5 & 1.5 & 3 & N & .7 & $\mathbb{N}$ \\
\hline WV0120R & 475259 & 92239 & 15 & 5 & 2 & .5 & $\mathrm{~N}$ & .7 & $<.5$ \\
\hline WV0121R & $4753 \quad 21$ & $92 \quad 2253$ & 10 & 7 & 1.5 & 1 & $\mathrm{~N}$ & .7 & $\mathrm{~N}$ \\
\hline WV0123R & $4753 \quad 0$ & 922348 & 7 & 5 & 3 & .7 & N & .3 & N \\
\hline WV0124R & $4753 \quad 0$ & $92 \quad 24 \quad 14$ & 7 & 5 & 1.5 & 1 & $\mathrm{~N}$ & .7 & N \\
\hline WV0127R & $4752 \quad 36$ & $92 \quad 2532$ & .3 & 3 & 1 & 3 & $<.2$ & .5 & .5 \\
\hline WV0128R & $4752 \quad 10$ & 922641 & .3 & 3 & 1.5 & 3 & $<.2$ & .7 & $N$ \\
\hline WV0129R & $4752 \quad 41$ & $92 \quad 2652$ & 3 & 5 & 2 & 1.5 & $<.2$ & .5 & N \\
\hline WV0133R & 475353 & $92 \quad 2859$ & 5 & 3 & 1.5 & 3 & $<.2$ & .7 & $\mathrm{~N}$ \\
\hline WV0145R & 475357 & $9232 \quad 26$ & .7 & .7 & .2 & 2 & $<.2$ & .03 & $<.5$ \\
\hline
\end{tabular}


Table 1. Gold and spectrographic analyses of 110 outcrop samples.--Continued

Sample As ppm-s B ppm-s Ba ppm-s Be ppm-s Bi ppm-s Cd ppm-s Co ppm-s Cr ppm-s Cu ppm-s

\begin{tabular}{|c|c|c|c|c|c|c|c|c|c|}
\hline WV0002R & N & N & 150 & 2 & N & N & N & N & $<5$ \\
\hline WV0004R & N & $<10$ & 1,500 & 1 & N & N & N & $<10$ & 10 \\
\hline WV0005R & N & $<10$ & 500 & 1 & N & N & 30 & 150 & 30 \\
\hline WV0006R & N & 20 & 300 & N & N & N & 50 & 150 & 50 \\
\hline WV0007R & $\mathbf{N}$ & 20 & 500 & 1 & $\mathbf{N}$ & N & 30 & 200 & 30 \\
\hline WV0009R & $\mathbf{N}$ & 20 & 700 & 1 & N & N & 30 & 150 & 30 \\
\hline WV0011R & $\mathbf{N}$ & 10 & 700 & 1 & $\mathbf{N}$ & N & 15 & 100 & 15 \\
\hline WV0012R1 & $N$ & 15 & 700 & $<1$ & $\mathrm{~N}$ & N & 20 & 300 & 20 \\
\hline WV0012R2 & $\mathbf{N}$ & 10 & 1,000 & 1.5 & $\mathbf{N}$ & $\mathrm{N}$ & $\mathbf{N}$ & 10 & 7 \\
\hline WV0013R & $N$ & 15 & 1,500 & $<1$ & $N$ & N & $<10$ & 50 & 5 \\
\hline WV0014R & N & $<10$ & 700 & 5 & N & $N$ & $\mathbf{N}$ & N & 5 \\
\hline WV0016R & N & $<10$ & 1,000 & $<1$ & N & N & 20 & 200 & 30 \\
\hline WV0017R & $\mathbf{N}$ & 10 & 1,000 & $<1$ & $\mathbf{N}$ & $\mathbf{N}$ & 30 & 300 & 50 \\
\hline WV0018R & $N$ & 15 & 700 & $<1$ & $\mathrm{~N}$ & $N$ & 30 & 700 & 50 \\
\hline WV0019R & $N$ & 15 & 700 & 1 & $N$ & $\mathbf{N}$ & 20 & 100 & 50 \\
\hline WVO021R & $N$ & 20 & 1,500 & $<1$ & N & $\mathrm{N}$ & 30 & 300 & 30 \\
\hline WV0022R & $N$ & $<10$ & 2,000 & $<1$ & $\mathbf{N}$ & $\mathrm{N}$ & 30 & 500 & 30 \\
\hline WV0023R & $\mathrm{N}$ & 20 & 1,000 & 1.5 & $\mathrm{~N}$ & $\mathrm{~N}$ & N & 15 & 7 \\
\hline WV0024R & $N$ & 20 & 500 & $<1$ & $\mathbf{N}$ & $\mathrm{N}$ & $<10$ & 50 & 15 \\
\hline WV0026R & $\mathrm{N}$ & $\mathrm{N}$ & 300 & N & $\mathbb{N}$ & $\mathrm{N}$ & 50 & 500 & 70 \\
\hline WV0027R & $N$ & N & 20 & $<1$ & $\mathbf{N}$ & $\mathbf{N}$ & $\mathrm{N}$ & 10 & $<5$ \\
\hline WW0029R & $N$ & 15 & 500 & $<1$ & $\mathbf{N}$ & $N$ & 20 & 100 & 20 \\
\hline WV0030R & $\mathbf{N}$ & $<10$ & 500 & 1 & $\mathbf{N}$ & $\mathbf{N}$ & 10 & 50 & 20 \\
\hline WV0030R & $N$ & 30 & 300 & 1 & N & $\mathrm{N}$ & $\mathrm{N}$ & 10 & 10 \\
\hline WV0032R & N & 50 & 300 & $<1$ & $\mathbf{N}$ & $\mathbb{N}$ & $<10$ & 30 & 10 \\
\hline WV0042R & $N$ & $<10$ & 500 & 1.5 & $\mathbf{N}$ & $\mathbf{N}$ & $\mathrm{N}$ & 10 & 10 \\
\hline WV0043R & $\mathbf{N}$ & N & 500 & $<1$ & $\mathbf{N}$ & $N$ & 15 & 150 & 15 \\
\hline WV0052R & $\mathbb{N}$ & 10 & 1,000 & $<1$ & $\mathbf{N}$ & $\mathbb{N}$ & 20 & 200 & 30 \\
\hline WV0055R & $\mathbf{N}$ & $N$ & 300 & $<1$ & $N$ & $\mathbb{N}$ & $N$ & N & 15 \\
\hline WV0056R & $N$ & $<10$ & 2,000 & 1 & $N$ & $N$ & 20 & 100 & 20 \\
\hline WV0068R & $\mathrm{N}$ & 30 & 700 & $<1$ & $\mathbf{N}$ & $\mathbb{N}$ & 20 & 150 & 30 \\
\hline WV0071R & $\mathrm{N}$ & 10 & 500 & 1.5 & $\mathrm{~N}$ & $\mathrm{~N}$ & $\mathrm{~N}$ & 20 & 7 \\
\hline WV0071R1 & $\mathbb{N}$ & $<10$ & 3,000 & 1 & $\mathrm{~N}$ & $\mathbf{N}$ & $<10$ & $<10$ & 20 \\
\hline WV0079R & $\mathbf{N}$ & 15 & 700 & $<1$ & N & N & 30 & 200 & 30 \\
\hline WV0112R & $\mathbf{N}$ & N & 2,000 & 1 & $\mathrm{~N}$ & $N$ & 30 & 200 & 15 \\
\hline WV0113R & $\mathbf{N}$ & $<10$ & 1,000 & 1.5 & $\mathrm{~N}$ & $\mathrm{~N}$ & 50 & 700 & 50 \\
\hline WV0114R & N & $\mathbb{N}$ & 1,500 & $<1$ & $N$ & $\mathbf{N}$ & 30 & 700 & 50 \\
\hline WV0116R & N & N & 70 & N & $\mathbb{N}$ & $\mathrm{N}$ & 70 & 300 & 50 \\
\hline WV0117R & N & 20 & 1,500 & 1 & $\mathrm{~N}$ & N & 30 & 150 & 50 \\
\hline WV0118R & $\mathrm{N}$ & $\mathbf{N}$ & 100 & N & $\mathrm{N}$ & $\mathrm{N}$ & 50 & 20 & 30 \\
\hline WV0119R & $\mathrm{N}$ & 50 & 1,000 & $<1$ & N & $\mathbf{N}$ & 50 & 300 & 50 \\
\hline WV0120R & $\mathbb{N}$ & 70 & 1,500 & 1 & N & $\mathrm{N}$ & 15 & 300 & 30 \\
\hline WV0121R & $N$ & 10 & 300 & $<1$ & $\mathrm{~N}$ & $\mathrm{~N}$ & 70 & 500 & 50 \\
\hline WV0123R & $N$ & $<10$ & 300 & $<1$ & N & $\mathbf{N}$ & 50 & 500 & 5 \\
\hline WV0124R & $N$ & $\mathbf{N}$ & 150 & $<1$ & $\mathbf{N}$ & $\mathrm{N}$ & 70 & 500 & 50 \\
\hline WV0127R & $\mathbf{N}$ & $\mathrm{N}$ & 2,000 & 1 & $\mathbf{N}$ & $\mathbf{N}$ & 20 & 150 & 50 \\
\hline WV0128R & N & 20 & 100 & 1.5 & $\mathrm{~N}$ & $\mathbf{N}$ & 20 & 200 & 30 \\
\hline WV0129R & $\mathbf{N}$ & 10 & 700 & 1.5 & $\mathbf{N}$ & $\mathbf{N}$ & 30 & 200 & 30 \\
\hline WV0133R & $\mathbf{N}$ & $<10$ & 100 & 1 & N & $\mathbf{N}$ & 15 & 100 & 10 \\
\hline WV0145R & $\mathbf{N}$ & 10 & 700 & 1 & N & $\mathbf{N}$ & $N$ & 10 & $<5$ \\
\hline
\end{tabular}


Table 1. Gold and spectrographic analyses of 110 outcrop samples.--Continued

Sample Ga ppm-s Ge ppm-s La ppm-s In ppm-s Mo ppm-s Nb ppm-s Ni ppm-s Pb ppm-s Sb ppm-s Sc ppm-s

\begin{tabular}{|c|c|c|c|c|c|c|c|c|c|c|}
\hline WV0002R & 30 & N & $<50$ & 150 & N & $<20$ & N & 70 & $\mathbb{N}$ & $<5$ \\
\hline WV0004R & 30 & N & N & 100 & $\mathbb{N}$ & N & N & 70 & $\mathbb{N}$ & $<5$ \\
\hline WV0005R & 30 & $\mathrm{~N}$ & $<50$ & 700 & N & $<20$ & 50 & $<10$ & $\mathbb{N}$ & 10 \\
\hline WV0006R & 50 & $\mathbb{N}$ & $\mathbf{N}$ & 1,000 & $\mathbb{N}$ & $<20$ & 50 & $<10$ & $\mathbb{N}$ & 30 \\
\hline WV0007R & 50 & $\mathbb{N}$ & $<50$ & 700 & N & $<20$ & 70 & 30 & $\mathbb{N}$ & 15 \\
\hline WV0009R & 50 & $\mathrm{~N}$ & $<50$ & 700 & N & $<20$ & 70 & 30 & $\mathbb{N}$ & 15 \\
\hline WV0011R & 50 & N & $<50$ & 500 & $\mathbb{N}$ & $<20$ & 30 & 20 & $\mathbb{N}$ & 10 \\
\hline WV0012R1 & 50 & $\mathbb{N}$ & $<50$ & 2,000 & $\mathbb{N}$ & $<20$ & 50 & 30 & $\mathbb{N}$ & 30 \\
\hline WV0012R2 & 30 & $N$ & $<50$ & 150 & N & N & $<5$ & 50 & $\mathbb{N}$ & $\mathbb{N}$ \\
\hline WV0013R & 50 & $\mathbb{N}$ & $<50$ & 500 & $<5$ & $<20$ & 20 & 30 & $N$ & $<5$ \\
\hline WV0014R & 50 & $\mathrm{~N}$ & $\mathbb{N}$ & 1,500 & N & $<20$ & 7 & 50 & $\mathbb{N}$ & $<5$ \\
\hline WV0016R & 50 & $\mathbb{N}$ & $<50$ & 700 & N & $<20$ & 70 & 30 & $N$ & 20 \\
\hline WW0017R & 70 & $\mathrm{~N}$ & $<50$ & 1,000 & N & $<20$ & 70 & 70 & N & 15 \\
\hline WV0018R & 50 & N & $<50$ & 700 & 7 & $<20$ & 150 & 30 & N & 15 \\
\hline WV0019R & 50 & $\mathrm{~N}$ & $<50$ & 700 & N & $<20$ & 30 & 20 & $\mathrm{~N}$ & 15 \\
\hline WV0021R & 50 & $N$ & 50 & 1,000 & N & $<20$ & 70 & 30 & N & 15 \\
\hline WV0022R & 50 & $\mathbb{N}$ & 50 & 1,000 & 10 & $<20$ & 150 & 30 & $\mathbb{N}$ & 20 \\
\hline WV0023R & 30 & $\mathrm{~N}$ & $<50$ & 150 & $N$ & $\mathbb{N}$ & 5 & 30 & $\mathbb{N}$ & $<5$ \\
\hline WV0024R & 50 & $\mathrm{~N}$ & $<50$ & 150 & N & N & 20 & 15 & $N$ & 5 \\
\hline WV0026R & 30 & $\mathbb{N}$ & $N$ & 1,500 & $N$ & N & 100 & $<10$ & $\mathbb{N}$ & 50 \\
\hline WV0027R & $\mathbb{N}$ & $\mathbb{N}$ & $\mathrm{N}$ & 30 & N & N & N & N & $\mathbb{N}$ & $\mathbb{N}$ \\
\hline WV0029R & 30 & $\mathrm{~N}$ & N & 300 & $\mathrm{~N}$ & N & 50 & 15 & $\mathbb{N}$ & 7 \\
\hline WV0030R & 50 & $\mathbb{N}$ & N & 700 & N & $<20$ & 30 & 10 & $\mathbb{N}$ & 5 \\
\hline WV0030R & 30 & $\mathrm{~N}$ & $\mathrm{~N}$ & 150 & N & N & 7 & 15 & $\mathbb{N}$ & N \\
\hline WV0032R & 50 & $\mathrm{~N}$ & N & 150 & N & $\mathbb{N}$ & 10 & $<10$ & $\mathrm{~N}$ & 5 \\
\hline WV0042R & 70 & $\mathrm{~N}$ & $<50$ & 150 & N & $<20$ & $\mathrm{~N}$ & 30 & $\mathrm{~N}$ & $<5$ \\
\hline WV0043R & 30 & $N$ & $<50$ & 500 & N & $<20$ & 50 & $<10$ & $\mathrm{~N}$ & 10 \\
\hline WV0052R & 50 & $\mathbb{N}$ & 50 & 1,000 & 5 & $<20$ & 50 & 30 & $\mathbb{N}$ & 15 \\
\hline WV0055R & 20 & N & N & 150 & $N$ & N & 7 & $<10$ & N & N \\
\hline WV0056R & 50 & $\mathrm{~N}$ & 150 & 500 & $N$ & 20 & 50 & 10 & $\mathbb{N}$ & 10 \\
\hline WV0068R & 50 & $\mathbb{N}$ & $<50$ & 700 & 50 & $<20$ & 50 & 70 & $\mathrm{~N}$ & 10 \\
\hline WV0071R & 30 & $\mathrm{~N}$ & N & 300 & $\mathrm{~N}$ & $<20$ & 7 & 10 & $\mathrm{~N}$ & $<5$ \\
\hline WV0071RI & 50 & $\mathrm{~N}$ & 200 & 500 & $<5$ & $<20$ & $<5$ & 70 & N & $<5$ \\
\hline WV0079R & 50 & $N$ & 50 & 1,000 & N & $<20$ & 70 & 20 & $\mathbb{N}$ & 15 \\
\hline WV0112R & 50 & $\mathrm{~N}$ & 50 & 700 & $\mathbb{N}$ & $<20$ & 70 & 50 & $\mathrm{~N}$ & 15 \\
\hline WV0113R & 30 & N & $<50$ & 1,000 & N & $<20$ & 100 & 20 & $\mathrm{~N}$ & 15 \\
\hline WV0114R & 30 & $\mathbf{N}$ & 50 & 1,000 & $N$ & N & 70 & 15 & N & 15 \\
\hline WV0116R & 30 & $\mathrm{~N}$ & $\mathrm{~N}$ & 1,500 & $N$ & $\mathbf{N}$ & 70 & $<10$ & N & 30 \\
\hline WV0117R & 50 & $\mathbb{N}$ & 50 & 700 & N & $<20$ & 70 & 20 & $\mathrm{~N}$ & 15 \\
\hline WV0118R & 50 & $\mathrm{~N}$ & N & 1,500 & $N$ & $<20$ & 30 & $<10$ & $N$ & 20 \\
\hline WV0119R & 70 & $N$ & 50 & 700 & $N$ & N & 100 & 15 & $\mathbb{N}$ & 20 \\
\hline WV0120R & 50 & $\mathrm{~N}$ & 50 & 1,000 & N & $<20$ & 50 & 70 & $\mathbb{N}$ & 15 \\
\hline WV0121R & 50 & $\mathbf{N}$ & N & 1,500 & N & N & 100 & 10 & $\mathrm{~N}$ & 30 \\
\hline WV0123R & 20 & $\mathbb{N}$ & $<50$ & 1,500 & $N$ & $\mathrm{~N}$ & 100 & $<10$ & $\mathrm{~N}$ & 15 \\
\hline WV0124R & 30 & $N$ & $\mathrm{~N}$ & 1,500 & $N$ & $\mathbb{N}$ & 100 & $<10$ & $\mathrm{~N}$ & 30 \\
\hline WV0127R & 50 & $\mathrm{~N}$ & $<50$ & 300 & N & $<20$ & 50 & 50 & $\mathrm{~N}$ & 10 \\
\hline WV0128R & 50 & $\mathbf{N}$ & 50 & 300 & $\mathbb{N}$ & $<20$ & 70 & 20 & N & 20 \\
\hline WV0129R & 50 & $\mathbb{N}$ & 50 & 700 & $\mathbb{N}$ & $\mathrm{N}$ & 50 & 15 & $\mathrm{~N}$ & 20 \\
\hline WN0133R & 50 & $\mathbf{N}$ & $<50$ & 1,000 & $\mathbb{N}$ & $<20$ & 30 & 20 & $\mathrm{~N}$ & 10 \\
\hline WV0145R & 30 & $\mathrm{~N}$ & $\mathrm{~N}$ & 500 & N & N & 5 & 10 & $\mathrm{~N}$ & $\mathrm{~N}$ \\
\hline
\end{tabular}


Table 1. Gold and spectrographic analyses of 110 outcrop samples.--Continued

Sample Sn ppm-s Sr ppm-s Th ppm-s V ppm-s W ppm-s Y ppm-s Zn ppm-s Ir ppm-s Au ppm-a

\begin{tabular}{|c|c|c|c|c|c|c|c|c|c|}
\hline$W V 0002 R$ & N & 200 & N & $<10$ & N & 20 & N & 70 & $<.001$ \\
\hline WV0004R & N & 300 & N & 15 & N & 15 & N & 20 & $<.001$ \\
\hline WV0005R & N & 300 & N & 70 & N & 15 & N & 150 & $<.001$ \\
\hline WV0006R & N & 150 & $\mathbf{N}$ & 200 & $N$ & 30 & $<200$ & 70 & $<.001$ \\
\hline WV0007R & N & 300 & N & 150 & N & 15 & $<200$ & 100 & $<.001$ \\
\hline WV0009R & N & 300 & $\mathrm{~N}$ & 150 & N & 15 & $<200$ & 150 & $<.001$ \\
\hline WV0011R & $\mathrm{N}$ & 500 & $N$ & 70 & $\mathbf{N}$ & $<10$ & N & 70 & $<.001$ \\
\hline WV0012R1 & N & 700 & $\mathbf{N}$ & 200 & $\mathbb{N}$ & 30 & N & 100 & $<.001$ \\
\hline WV0012R2 & $N$ & 300 & $N$ & 15 & $\mathbf{N}$ & $<10$ & N & 50 & $<.001$ \\
\hline WV0013R & $\mathrm{N}$ & 700 & $N$ & 50 & $N$ & $<10$ & N & 100 & $<.001$ \\
\hline WV0014R & $\mathbf{N}$ & 150 & N & $<10$ & $\mathbf{N}$ & 20 & N & 50 & $<.001$ \\
\hline WV0016R & $N$ & 500 & $N$ & 150 & $N$ & 20 & $N$ & 100 & $<.001$ \\
\hline WN0017R & $\mathbf{N}$ & 1,000 & N & 100 & $\mathrm{~N}$ & 20 & $<200$ & 150 & .001 \\
\hline WO0018R & $\mathbf{N}$ & 700 & $N$ & 150 & $\mathbb{N}$ & 15 & N & 500 & $<.001$ \\
\hline WV0019R & $\mathbf{N}$ & 700 & $N$ & 150 & $\mathrm{~N}$ & 15 & N & 70 & $<.001$ \\
\hline WW0021R & $\mathbf{N}$ & 1,000 & $\mathbf{N}$ & 150 & $\mathbf{N}$ & 20 & N & 200 & $<.001$ \\
\hline WN0022R & $\mathbf{N}$ & 500 & $\mathrm{~N}$ & 200 & $\mathbf{N}$ & 30 & $\mathbb{N}$ & 150 & $<.001$ \\
\hline WW0023R & $N$ & 700 & $\mathrm{~N}$ & 20 & $\mathbf{N}$ & $<10$ & N & 70 & $<.001$ \\
\hline WV0024R & $N$ & 500 & $\mathrm{~N}$ & 50 & $\mathbb{N}$ & 10 & $\mathbf{N}$ & 70 & .003 \\
\hline WV0026R & $\mathbf{N}$ & 150 & N & 300 & $\mathrm{~N}$ & 30 & N & 70 & .001 \\
\hline WV0027R & 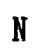 & N & $\mathrm{N}$ & $<10$ & $\mathbf{N}$ & N & $\mathrm{N}$ & 10 & $<.001$ \\
\hline WV0029R & $N$ & 200 & $\mathbb{N}$ & 70 & $N$ & $<10$ & $\mathbf{N}$ & 100 & $<.001$ \\
\hline WVO030R & $N$ & 300 & $\mathrm{~N}$ & 70 & $\mathbf{N}$ & $<10$ & $\mathbb{N}$ & 70 & $<.001$ \\
\hline WVO030R & $N$ & 300 & N & 15 & $N$ & $N$ & $\mathrm{~N}$ & 50 & $<.001$ \\
\hline WV0032R & $\mathrm{N}$ & 300 & N & 30 & $\mathbf{N}$ & $<10$ & $\mathrm{~N}$ & 100 & $<.001$ \\
\hline WV0042R & $N$ & 300 & $N$ & 30 & $N$ & 10 & $N$ & 300 & $<.001$ \\
\hline WV0043R & $\mathbf{N}$ & 300 & $N$ & 100 & $\mathbf{N}$ & 10 & N & 200 & $<.001$ \\
\hline WV0052R & $\mathbf{N}$ & 500 & $N$ & 150 & $N$ & 20 & N & 200 & $<.001$ \\
\hline WV0055R & $\mathrm{N}$ & N & $\mathbf{N}$ & 20 & $\mathbf{N}$ & N & N & 70 & $<.001$ \\
\hline WV0056R & $\mathrm{N}$ & 500 & $\mathbf{N}$ & 70 & $\mathbf{N}$ & 30 & N & 200 & $<.001$ \\
\hline WV0068R & $\mathbf{N}$ & 300 & $N$ & 100 & N & 10 & N & 70 & $<.001$ \\
\hline WV0071R & $\mathrm{N}$ & 300 & $\mathrm{~N}$ & 30 & $\mathrm{~N}$ & $<10$ & N & 70 & $<.001$ \\
\hline W0071R1 & $\mathbf{N}$ & 2,000 & $\mathbb{N}$ & 30 & $\mathrm{~N}$ & 20 & N & 300 & $<.001$ \\
\hline WV0079R & $\mathbf{N}$ & 1,000 & N & 150 & $\mathrm{~N}$ & 20 & $\mathrm{~N}$ & 200 & $<.001$ \\
\hline WV0112R & $\mathrm{N}$ & 700 & $\mathbb{N}$ & 150 & N & 20 & $<200$ & 200 & $<.001$ \\
\hline WV0113R & $N$ & 1,000 & $N$ & 150 & $N$ & 20 & $<200$ & 150 & .003 \\
\hline WV0114R & $\mathbf{N}$ & 2,000 & $\mathrm{~N}$ & 150 & $\mathrm{~N}$ & 20 & $\mathbf{N}$ & 30 & .01 \\
\hline WV0116R & $N$ & 100 & $\mathrm{~N}$ & 200 & $N$ & 30 & $<200$ & 50 & .001 \\
\hline WV0117R & $\mathrm{N}$ & 500 & $\mathbf{N}$ & 150 & $\mathbf{N}$ & 20 & $\mathbf{N}$ & 200 & .001 \\
\hline WV0118R & $N$ & 150 & $\mathbb{N}$ & 300 & $N$ & 50 & $<200$ & 150 & $<.001$ \\
\hline WV0119R & $\mathbf{N}$ & 200 & $\mathrm{~N}$ & 200 & $\mathrm{~N}$ & 20 & $<200$ & 200 & .003 \\
\hline WV0120R & $\mathbf{N}$ & 700 & $\mathrm{~N}$ & 150 & $\mathrm{~N}$ & 20 & $\mathrm{~N}$ & 150 & $<.001$ \\
\hline WV0121R & N & 500 & $N$ & 300 & $\mathbf{N}$ & 30 & $<200$ & 50 & $<.001$ \\
\hline WV0123R & $\mathbf{N}$ & 1,000 & $\mathrm{~N}$ & 150 & $\mathbf{N}$ & 20 & $<200$ & 50 & $<.001$ \\
\hline WV0124R & N & 300 & $\mathrm{~N}$ & 300 & $\mathbf{N}$ & 30 & $<200$ & 50 & .002 \\
\hline WV0127R & $\mathrm{N}$ & 500 & $\mathrm{~N}$ & 150 & $\mathrm{~N}$ & 15 & $<200$ & 100 & .002 \\
\hline WN0128R & $\mathbf{N}$ & 300 & N & 200 & $\mathrm{~N}$ & 30 & $<200$ & 150 & .001 \\
\hline WV0129R & $N$ & 1,000 & N & 150 & N & 15 & $\mathrm{~N}$ & 150 & $<.001$ \\
\hline WV0133R & N & 700 & N & 150 & $\mathbf{N}$ & 15 & $\mathrm{~N}$ & 150 & $<.001$ \\
\hline WV0145R & $N$ & 300 & N & 15 & $N$ & $<10$ & N & 50 & $<.001$ \\
\hline
\end{tabular}


Table 1. Gold and spectrographic analyses of 110 outcrop samples.--Continued

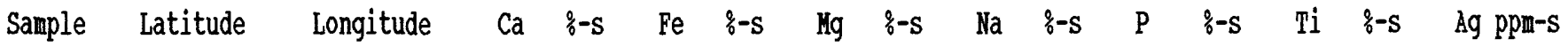

\begin{tabular}{|c|c|c|c|c|c|c|c|c|c|}
\hline WN0149R & $4754 \quad 25$ & 923423 & .3 & .7 & .5 & 2 & $<.2$ & .07 & N \\
\hline WV0150R & $4754 \quad 15$ & $9235 \quad 6$ & 1 & 2 & .7 & 2 & $<.2$ & .3 & $\mathbb{N}$ \\
\hline WV0177R & $4755 \quad 14$ & 923926 & 3 & 3 & 1.5 & 2 & $<.2$ & .5 & $\mathbb{N}$ \\
\hline WV0184R & 475157 & $9241 \quad 12$ & 7 & 5 & 2 & 3 & $\mathrm{~N}$ & .5 & N \\
\hline WV0202R & 475421 & $92 \quad 2757$ & 1 & 3 & 1 & 1.5 & $<.2$ & .5 & .7 \\
\hline WV0203R & 475358 & $92 \quad 2821$ & .7 & 1 & .3 & 2 & $<.2$ & .15 & N \\
\hline WV0204R & $4753 \quad 35$ & $92 \quad 2811$ & .7 & 1.5 & .3 & 3 & $<.2$ & .15 & N \\
\hline WV0205R & $4753 \quad 48$ & 922738 & .7 & 1.5 & .5 & 3 & $<.2$ & .15 & $\mathbb{N}$ \\
\hline WV0206R1 & $4754 \quad 1$ & $92 \quad 27 \quad 17$ & .7 & 1 & .3 & 2 & $<.2$ & .07 & $<.5$ \\
\hline WV0206R2 & $4754 \quad 1$ & $92 \quad 27 \quad 17$ & 1.5 & 5 & 1.5 & 2 & $N$ & .5 & $N$ \\
\hline WV0207R & $4753 \quad 31$ & $9227 \quad 1$ & 2 & 3 & 1 & 1.5 & $<.2$ & .5 & .5 \\
\hline WV0208R & $4753 \quad 40$ & 922646 & .15 & 1.5 & .7 & 2 & $<.2$ & .15 & $\mathbb{N}$ \\
\hline WV0209R & $4753 \quad 13$ & 922722 & 2 & 3 & 1.5 & 2 & $\mathrm{~N}$ & .5 & 2 \\
\hline WV0210R & 475257 & 922820 & 1.5 & 3 & 1.5 & 1.5 & $<.2$ & .5 & $N$ \\
\hline WV0211R & $47 \quad 53 \quad 13$ & $92 \quad 28 \quad 23$ & .7 & 3 & 1.5 & 1.5 & $<.2$ & .3 & $\mathrm{~N}$ \\
\hline WW0212R & $4753 \quad 2$ & 922935 & .7 & 3 & 2 & 2 & .2 & .5 & N \\
\hline WV0216R & $4752 \quad 3$ & $92 \quad 2715$ & 3 & 5 & 1.5 & 1 & N & .5 & $\mathrm{~N}$ \\
\hline WV0218R1 & $4752 \quad 24$ & 922755 & 2 & 1.5 & .7 & 1.5 & $<.2$ & .07 & $\mathrm{~N}$ \\
\hline WV0218R2 & $4752 \quad 24$ & 922755 & .2 & 5 & 1 & 2 & .2 & .5 & $N$ \\
\hline WV0219R & $4753 \quad 16$ & 922636 & 7 & 7 & 2 & 1.5 & $\mathrm{~N}$ & 1 & 5 \\
\hline WVO220R & 475334 & $92 \quad 2515$ & 7 & 5 & 1.5 & 2 & N & .3 & .5 \\
\hline WV0221R & 475339 & 922433 & 7 & 7 & 3 & 1.5 & N & .5 & $\mathbb{N}$ \\
\hline WV0222R & $4749 \quad 8$ & 922655 & 1.5 & 5 & 2 & 5 & $<.2$ & .5 & $\mathbb{N}$ \\
\hline WV0222R1 & $4749 \quad 8$ & 922655 & .15 & .2 & .2 & $<.2$ & $<.2$ & .03 & $N$ \\
\hline WV0222R2 & $4749 \quad 8$ & 922655 & 2 & 3 & 2 & 3 & $<.2$ & .5 & $N$ \\
\hline WV0224R & 474924 & 92247 & .7 & 3 & 1.5 & 3 & $<.2$ & .3 & $<.5$ \\
\hline WV0225R & $47 \quad 49 \quad 42$ & 922413 & 1 & 5 & 2 & 2 & N & .5 & $\mathrm{~N}$ \\
\hline WV0227R & 474956 & 922451 & 5 & 5 & 2 & 5 & N & 1 & $N$ \\
\hline WV0229R & $4754 \quad 17$ & 923229 & 7 & 2 & 1 & 5 & N & .3 & N \\
\hline WV0234R & 475541 & 923046 & .7 & .7 & .3 & 3 & $<.2$ & .07 & $N$ \\
\hline WV0241R & $4754 \quad 28$ & 923330 & .15 & .5 & .15 & 2 & $<.2$ & .05 & $<.5$ \\
\hline WV0242R & $4754 \quad 41$ & 923359 & 1.5 & 1.5 & .3 & 2 & $<.2$ & .2 & 10 \\
\hline WV0243R & $4751 \quad 25$ & $92 \quad 1611$ & .7 & 1.5 & .5 & 2 & $<.2$ & .2 & .5 \\
\hline WV0244R & 475118 & $9216 \quad 0$ & 1.5 & 1.5 & .7 & 5 & $<.2$ & .3 & .5 \\
\hline WV0245R & 475114 & 922556 & 2 & 1.5 & 1 & 3 & $<.2$ & .3 & 3 \\
\hline WV0246R & $4751 \quad 17$ & 92164 & 5 & 2 & 1.5 & 5 & $N$ & .5 & 1 \\
\hline WN0247R & 475124 & $9216 \quad 6$ & .05 & 10 & .3 & 2 & $N$ & .07 & $N$ \\
\hline WV0248R1 & 475110 & 921611 & 1.5 & .7 & .2 & 1.5 & $<.2$ & .07 & $<.5$ \\
\hline WV0248R2 & 475110 & 921611 & 1 & .7 & .2 & 1.5 & $N$ & .07 & 15 \\
\hline WV0249R & 475134 & 921136 & 7 & 5 & 5 & 2 & $\mathbb{N}$ & .5 & $\mathbb{N}$ \\
\hline WVO250R & 475131 & 921140 & 7 & 5 & 2 & 1.5 & N & 1 & N \\
\hline WV251R & 475133 & 921137 & 5 & 5 & 2 & 2 & N & .7 & $\mathbb{N}$ \\
\hline WV252R1 & 475123 & 921131 & .7 & .7 & .5 & 2 & $<.2$ & .15 & N \\
\hline WV0252R2 & $4751 \quad 23$ & 921131 & .5 & .15 & .03 & $<.2$ & $<.2$ & .015 & $<.5$ \\
\hline WV0253R & $4751 \quad 17$ & 921130 & 3 & 1.5 & .7 & 3 & $<.2$ & .3 & $N$ \\
\hline WN0254R & 475140 & 921143 & .7 & 1 & .5 & 2 & $<.2$ & .2 & $N$ \\
\hline WVO255R & 475148 & 921132 & .7 & 1 & .5 & 2 & $<.2$ & .2 & N \\
\hline WV0256R & $4751 \quad 47$ & 92116 & .2 & 1.5 & .7 & 2 & $<.2$ & .2 & $\mathrm{~N}$ \\
\hline WVO257R & 475154 & 921145 & .7 & 3 & 1.5 & 2 & $<.2$ & .5 & $<.5$ \\
\hline WVO258R & $4752 \quad 6$ & 921122 & .1 & 3 & 1 & 1.5 & $<.2$ & .5 & $N$ \\
\hline
\end{tabular}


Table 1. Gold and spectrographic analyses of 110 outcrop samples.--Continued

Sample As ppm-s B ppm-s Ba ppm-s Be ppm-s Bi ppm-s Cd ppm-s Co ppm-s Cr ppm-s Cu ppm-s

\begin{tabular}{|c|c|c|c|c|c|c|c|c|c|}
\hline WV0149R & $N$ & 15 & 100 & $<1$ & N & N & $N$ & $<10$ & $N$ \\
\hline WV0150R & $\mathbb{N}$ & 20 & 700 & $<1$ & N & N & 10 & 15 & 7 \\
\hline WV0177R & $\mathrm{N}$ & $<10$ & 700 & 1 & $N$ & $N$ & 20 & 300 & 30 \\
\hline WV0184R & $\mathbf{N}$ & 10 & 1,000 & $<1$ & $N$ & N & 20 & 700 & 20 \\
\hline WV0202R & $\mathrm{N}$ & $<10$ & 500 & 1 & N & $N$ & 15 & 150 & 20 \\
\hline WV0203R & $N$ & 20 & 1,000 & 1.5 & $N$ & $N$ & N & 15 & 5 \\
\hline WV0204R & $N$ & 15 & 1,000 & 2 & N & N & $\mathrm{N}$ & 10 & $<5$ \\
\hline WV0205R & N & 10 & 1,500 & 1 & N & N & N & 15 & $<5$ \\
\hline WV0206R1 & $N$ & $<10$ & 1,500 & 2 & N & N & $\mathbf{N}$ & 15 & 5 \\
\hline WV0206R2 & $\mathrm{N}$ & $<10$ & 700 & 1.5 & N & N & 30 & 500 & 50 \\
\hline WV0207R & $N$ & $<10$ & 700 & 1 & $\mathrm{~N}$ & $N$ & 20 & 10 & 20 \\
\hline WV0208R & $N$ & 10 & 1,000 & 1 & $N$ & N & $<10$ & 30 & 20 \\
\hline WV0209R & $\mathbb{N}$ & 15 & 1,000 & 1 & $N$ & N & 15 & 200 & 30 \\
\hline WV0210R & N & 10 & 1,000 & 1 & $\mathrm{~N}$ & $\mathrm{~N}$ & 20 & 200 & 30 \\
\hline WV0211R & $N$ & $<10$ & 700 & $<1$ & $\mathrm{~N}$ & $\mathrm{~N}$ & 20 & 150 & 20 \\
\hline WV0212R & $N$ & 10 & 700 & 1.5 & $\mathbb{N}$ & N & 20 & 70 & 5 \\
\hline WV0216R & $N$ & $<10$ & 300 & N & $\mathrm{N}$ & $\mathrm{N}$ & 70 & 300 & 50 \\
\hline WV0218R1 & $\mathbb{N}$ & $<10$ & 300 & $<1$ & $\mathrm{~N}$ & $N$ & N & 30 & 30 \\
\hline WV0218R2 & $N$ & 15 & 700 & 1 & $\mathbf{N}$ & $N$ & 30 & 150 & 50 \\
\hline WV0219R & $N$ & $\mathbb{N}$ & 150 & $<1$ & $N$ & N & 50 & 300 & 100 \\
\hline WV0220R & N & N & 20 & $\mathrm{~N}$ & $\mathrm{~N}$ & N & 30 & 300 & 50 \\
\hline WV0221R & $N$ & $N$ & 300 & N & N & $\mathrm{N}$ & 70 & 1,500 & 30 \\
\hline WV0222R & $\mathrm{N}$ & 20 & 1,500 & 1 & $\mathrm{~N}$ & N & 20 & 200 & 30 \\
\hline WV0222RI & $N$ & N & 150 & $<1$ & $\mathrm{~N}$ & N & N & 10 & N \\
\hline WV0222R2 & $\mathbb{N}$ & 20 & 1,000 & $<1$ & $N$ & $N$ & 20 & 150 & 50 \\
\hline WW0224R & $N$ & 20 & 700 & $<1$ & $\mathrm{~N}$ & N & 30 & 150 & 150 \\
\hline WV0225R & $N$ & 30 & 700 & $<1$ & $\mathbf{N}$ & N & 20 & 200 & 30 \\
\hline WV0227R & $\mathbf{N}$ & 20 & 1,500 & $<1$ & $\mathbf{N}$ & N & 20 & 300 & 30 \\
\hline WVO229R & $N$ & 50 & 1,500 & $<1$ & N & N & $N$ & 20 & 10 \\
\hline WV0234R & $N$ & 30 & 200 & 1 & N & N & N & $<10$ & 10 \\
\hline WV0241R & $N$ & 10 & 200 & 1.5 & N & N & $\mathrm{N}$ & N & 5 \\
\hline WV0242R & $N$ & 10 & 300 & 1 & N & N & $\mathrm{N}$ & 10 & 15 \\
\hline WV0243R & $N$ & 15 & 700 & 1 & N & N & $\mathrm{N}$ & 20 & 100 \\
\hline WV0244R & N & 30 & 1,000 & $<1$ & N & N & 20 & 50 & 700 \\
\hline WV0245R & $\mathrm{N}$ & 50 & 1,000 & 1 & N & $\mathrm{N}$ & 10 & 30 & 200 \\
\hline WV0246R & $N$ & 30 & 1,000 & $<1$ & N & N & $<10$ & 70 & 150 \\
\hline WV0247R & N & 20 & 200 & $<1$ & $N$ & $\mathbb{N}$ & $<10$ & 15 & 300 \\
\hline WV0248R1 & $\mathrm{N}$ & $\mathrm{N}$ & 70 & $<1$ & $N$ & N & $N$ & 15 & 7 \\
\hline WV0248R2 & $N$ & 10 & 300 & $<1$ & 15 & N & $N$ & 15 & 20 \\
\hline WV0249R & $\mathrm{N}$ & $\mathrm{N}$ & 1,000 & $<1$ & $\mathrm{~N}$ & N & 50 & 1,000 & 30 \\
\hline WV0250R & N & $\mathrm{N}$ & 150 & N & $\mathbb{N}$ & N & 70 & 500 & 50 \\
\hline WV0251R & $N$ & $<10$ & 300 & N & $\mathrm{N}$ & N & 70 & 500 & 70 \\
\hline WV0252R1 & $N$ & 30 & 700 & 1 & $\mathbf{N}$ & N & $N$ & 15 & N \\
\hline WV0252R2 & $N$ & $\mathrm{~N}$ & 150 & $<1$ & $\mathrm{~N}$ & N & N & 15 & 10 \\
\hline WV0253R & $\mathrm{N}$ & 20 & 500 & 1 & $\mathrm{~N}$ & $\mathrm{~N}$ & $N$ & 20 & $<5$ \\
\hline WV0254R & $\mathrm{N}$ & 30 & 700 & $<1$ & $\mathrm{~N}$ & N & $<10$ & 15 & $\mathbf{N}$ \\
\hline WV0255R & $\mathrm{N}$ & 30 & 700 & 1 & $\mathrm{~N}$ & $\mathrm{~N}$ & $<10$ & 15 & N \\
\hline WV0256R & $\mathbf{N}$ & 30 & 500 & $<1$ & $\mathrm{~N}$ & $\mathrm{~N}$ & $N$ & 30 & 5 \\
\hline WV0257R & $N$ & 50 & 1,000 & 1 & $\mathrm{~N}$ & $N$ & 20 & 200 & 50 \\
\hline WV0258R & $N$ & 30 & 700 & 1 & $\mathrm{~N}$ & $\mathbf{N}$ & 30 & 150 & 30 \\
\hline
\end{tabular}


Table 1. Gold and spectrographic analyses of 110 outcrop samples.--Continued

Sample Ga ppm-s Ge ppm-s La ppm-s In ppm-s Mo ppm-s Mb ppm-s Ni ppm-s Pb ppm-S Sb ppm-s Sc ppm-S

\begin{tabular}{|c|c|c|c|c|c|c|c|c|c|c|}
\hline WV0149R & 30 & N & N & 500 & N & N & $<5$ & 10 & N & N \\
\hline WV0150R & 50 & N & $<50$ & 500 & N & N & 7 & $<10$ & $\mathbb{N}$ & $\mathrm{N}$ \\
\hline WV0177R & 50 & N & $<50$ & 1,000 & N & $<20$ & 50 & 30 & N & 15 \\
\hline WV0184R & 50 & N & N & 1,500 & $N$ & $\mathrm{~N}$ & 100 & 20 & $N$ & 10 \\
\hline WV0202R & 30 & N & $N$ & 700 & N & N & 20 & 15 & $\mathrm{~N}$ & 10 \\
\hline WV0203R & 50 & N & $<50$ & 300 & N & N & 7 & 20 & $N$ & $<5$ \\
\hline WV0204R & 70 & N & $<50$ & 300 & N & $\mathrm{N}$ & 5 & 70 & N & $<5$ \\
\hline WV0205R & 70 & N & $<50$ & 500 & N & N & 7 & 70 & $\mathbf{N}$ & $<5$ \\
\hline WV206Rl & 50 & N & $<50$ & 500 & N & N & 5 & 70 & N & $<5$ \\
\hline WV0206R2 & 50 & N & N & 700 & $N$ & $<20$ & 100 & 30 & $\mathrm{~N}$ & 20 \\
\hline WV0207R & 30 & $\mathbb{N}$ & $<50$ & 700 & $\mathbb{N}$ & $N$ & 15 & 20 & $\mathbf{N}$ & $<5$ \\
\hline WV0208R & 50 & N & $<50$ & 700 & $N$ & N & 10 & 20 & $N$ & $<5$ \\
\hline WV0209R & 50 & N & $<50$ & 1,000 & 5 & $<20$ & 50 & 70 & N & 15 \\
\hline WV0210R & 50 & N & $<50$ & 700 & $<5$ & N & 70 & 20 & N & 15 \\
\hline WV0211R & 30 & N & $N$ & 700 & N & $N$ & 50 & 15 & N & 10 \\
\hline WV0212R & 50 & N & $<50$ & 500 & N & N & 30 & 10 & N & 7 \\
\hline WV0216R & 30 & N & N & 1,000 & N & N & 70 & N & $\mathbf{N}$ & 30 \\
\hline WV0218R1 & 10 & $N$ & $\mathrm{~N}$ & 1,500 & N & N & 15 & 10 & $\mathbf{N}$ & $<5$ \\
\hline WV0218R2 & 50 & N & $<50$ & 1,000 & N & $\mathrm{N}$ & 70 & 10 & N & 10 \\
\hline WV0219R & 30 & N & $N$ & 1,500 & N & N & 70 & $<10$ & $\mathbf{N}$ & 30 \\
\hline WV0220R & 30 & N & N & 1,500 & N & N & 70 & 10 & N & 30 \\
\hline WV0221R & 30 & N & N & 1,500 & N & N & 150 & $<10$ & N & 30 \\
\hline WV0222R & 70 & $N$ & 50 & 700 & N & $<20$ & 70 & 20 & N & 15 \\
\hline WO0222R1 & $<5$ & N & N & 30 & N & N & $<5$ & N & N & N \\
\hline WV0222R2 & 50 & $\mathrm{~N}$ & $<50$ & 700 & N & $<20$ & 70 & 15 & N & 15 \\
\hline WV0224R & 50 & N & $<50$ & 500 & N & N & 70 & 150 & $\mathbf{N}$ & 15 \\
\hline WV0225R & 50 & N & $<50$ & 700 & N & $<20$ & 70 & 20 & N & 15 \\
\hline WV0227R & 70 & N & 50 & 1,000 & $<5$ & $<20$ & 50 & 30 & N & 15 \\
\hline WO229R & 70 & $N$ & $<50$ & 1,000 & N & N & N & 100 & N & 7 \\
\hline WV0234R & 50 & $N$ & N & 300 & N & $N$ & $<5$ & 15 & N & $<5$ \\
\hline WV0241R & 50 & N & N & 700 & N & $N$ & $<5$ & 70 & N & 7 \\
\hline WV0242R & 50 & N & $<50$ & 500 & N & $\mathrm{N}$ & $<5$ & $<10$ & N & $\mathrm{N}$ \\
\hline WV0243R & 50 & N & $<50$ & 150 & 20 & N & 10 & $<10$ & $\mathrm{~N}$ & $<5$ \\
\hline WV0244R & 70 & N & $<50$ & 150 & 15 & N & 7 & $<10$ & N & 5 \\
\hline WV0245R & 50 & N & N & 500 & 10 & $N$ & 10 & 30 & N & $<5$ \\
\hline WV0246R & 50 & N & $<50$ & 500 & $\mathrm{~N}$ & N & 10 & 30 & N & 5 \\
\hline WV0247R & 50 & N & N & 20 & 10 & N & 20 & $<10$ & N & $<5$ \\
\hline WV0248RI & 7 & $\mathbb{N}$ & $N$ & 150 & $N$ & $N$ & 5 & $\mathbb{N}$ & N & $\mathbb{N}$ \\
\hline WV0248R2 & 5 & N & N & 150 & $N$ & $\mathbb{N}$ & $<5$ & 700 & N & $\mathrm{N}$ \\
\hline WV0249R & 50 & $\mathbb{N}$ & $<50$ & 1,000 & N & $N$ & 150 & $<10$ & N & 30 \\
\hline WVO250R & 50 & N & N & 1,500 & N & $<20$ & 100 & $<10$ & N & 50 \\
\hline WV0251R & 50 & N & N & 1,500 & $\mathrm{~N}$ & $N$ & 100 & $<10$ & N & 50 \\
\hline WVO252R1 & 30 & N & N & 300 & N & N & 5 & $\mathbf{N}$ & N & $<5$ \\
\hline WV0252R2 & N & $\mathrm{N}$ & N & 150 & $\mathrm{~N}$ & N & $<5$ & $\mathrm{~N}$ & N & $\mathrm{N}$ \\
\hline WV0253R & 50 & N & N & 200 & $N$ & $N$ & 7 & 15 & N & $<5$ \\
\hline WV0254R & 30 & $\mathrm{~N}$ & $<50$ & 500 & $N$ & N & 7 & 10 & N & $\mathrm{N}$ \\
\hline WV255R & 30 & N & $<50$ & 200 & 15 & N & 10 & 10 & $\mathrm{~N}$ & $<5$ \\
\hline WN0256R & 30 & $\mathrm{~N}$ & $<50$ & 300 & N & $\mathrm{N}$ & 15 & 20 & N & 5 \\
\hline WV0257R & 50 & $N$ & 50 & 700 & $N$ & $<20$ & 50 & 15 & N & 20 \\
\hline WV0258R & 50 & $N$ & 50 & 500 & N & $<20$ & 50 & 10 & N & 15 \\
\hline
\end{tabular}


Table 1. Gold and spectrographic analyses of 110 outcrop samples.--Continued

Sample Sn ppm-s Sr ppm-s Th ppm-s V ppm-s W ppm-s Y ppm-s Zn ppm-s Ir ppm-s Au ppm-a

\begin{tabular}{|c|c|c|c|c|c|c|c|c|c|}
\hline WV0149R & N & 300 & $\mathrm{~N}$ & 15 & N & $\mathbb{N}$ & $\mathbb{N}$ & 50 & $<.001$ \\
\hline WV0150R & N & 500 & $\mathbb{N}$ & 70 & N & N & $\mathrm{N}$ & 150 & $<.001$ \\
\hline WV0177R & N & 500 & $N$ & 150 & N & 20 & $N$ & 200 & $<.001$ \\
\hline WV0184R & N & 500 & $\mathrm{~N}$ & 150 & $N$ & 20 & $\mathbb{N}$ & 150 & $<.001$ \\
\hline WV0202R & $\mathbb{N}$ & 300 & $N$ & 100 & N & 10 & N & 70 & $<.001$ \\
\hline WV0203R & $N$ & 700 & $N$ & 30 & $N$ & $N$ & $N$ & 70 & $<.001$ \\
\hline WV0204R & N & 700 & $\mathbf{N}$ & 20 & $N$ & $<10$ & $\mathrm{~N}$ & 100 & $<.001$ \\
\hline WV0205R & $N$ & 500 & $N$ & 30 & $\mathbf{N}$ & 10 & N & 100 & $<.001$ \\
\hline WV0206R1 & $N$ & 300 & $N$ & 20 & $N$ & 20 & $N$ & 50 & $<.001$ \\
\hline WV0206R2 & $\mathbb{N}$ & 500 & $N$ & 150 & $\mathrm{~N}$ & 15 & N & 300 & $<.001$ \\
\hline WV0207R & $N$ & 500 & $N$ & 150 & $N$ & $<10$ & $N$ & 50 & .001 \\
\hline WV0208R & $\mathrm{N}$ & 200 & $\mathrm{~N}$ & 70 & $\mathrm{~N}$ & $<10$ & N & 50 & $<.001$ \\
\hline WV0209R & $N$ & 500 & $\mathrm{~N}$ & 150 & N & 15 & $\mathbb{N}$ & 200 & $<.001$ \\
\hline WV0210R & $N$ & 300 & $N$ & 150 & $N$ & 15 & $<200$ & 100 & $<.001$ \\
\hline WV0211R & $N$ & 200 & $\mathrm{~N}$ & 150 & $\mathrm{~N}$ & 10 & $\mathrm{~N}$ & 70 & $<.001$ \\
\hline WV0212R & $N$ & 500 & $\mathbf{N}$ & 150 & $N$ & 10 & $<200$ & 150 & $<.001$ \\
\hline WV0216R & $N$ & 150 & $N$ & 300 & $N$ & 30 & $<200$ & 70 & .001 \\
\hline WV0218R1 & $N$ & 300 & $N$ & 50 & N & 10 & $\mathbb{N}$ & 20 & .001 \\
\hline WV0218R2 & N & $<100$ & $N$ & 150 & N & 15 & $\mathrm{~N}$ & 200 & .004 \\
\hline WV219R & $N$ & 150 & N & 500 & $N$ & 30 & $<200$ & 70 & $<.001$ \\
\hline WW0220R & $N$ & 150 & $\mathrm{~N}$ & 200 & N & 20 & $\mathbb{N}$ & 30 & .002 \\
\hline WV0221R & $N$ & 200 & $N$ & 300 & $N$ & 20 & N & 30 & $<.001$ \\
\hline WV0222R & $N$ & 1,000 & $\mathrm{~N}$ & 150 & $\mathrm{~N}$ & 15 & $\mathrm{~N}$ & 200 & $<.001$ \\
\hline WV0222R1 & $N$ & N & $\mathrm{N}$ & 15 & $N$ & $N$ & $\mathrm{~N}$ & 10 & $<.001$ \\
\hline WV0222R2 & N & 500 & $N$ & 150 & $N$ & 15 & N & 150 & $<.001$ \\
\hline WV0224R & $N$ & 300 & $\mathrm{~N}$ & 150 & $\mathbb{N}$ & 15 & $<200$ & 150 & .003 \\
\hline WV0225R & $N$ & 700 & $N$ & 200 & $\mathbb{N}$ & 15 & $<200$ & 200 & .001 \\
\hline WV0227R & $N$ & 2,000 & $\mathbb{N}$ & 200 & $N$ & 20 & N & 150 & .001 \\
\hline WV0229R & $\mathrm{N}$ & $>5,000$ & $N$ & 70 & $N$ & 15 & N & 200 & $<.001$ \\
\hline WV0234R & $\mathrm{N}$ & 1,000 & N & 20 & $\mathbb{N}$ & N & N & 70 & $<.001$ \\
\hline WV0241R & $\mathrm{N}$ & $N$ & $\mathbb{N}$ & $<10$ & $N$ & 20 & $N$ & 50 & $<.001$ \\
\hline WV0242R & $N$ & 300 & $N$ & 30 & $N$ & $<10$ & N & 70 & $<.001$ \\
\hline WV0243R & $N$ & 200 & $N$ & 70 & $N$ & $N$ & $N$ & 100 & .48 \\
\hline WV0244R & $N$ & 700 & $\mathbb{N}$ & 30 & $N$ & $<10$ & $\mathrm{~N}$ & 100 & .028 \\
\hline WV0245R & N & 300 & $N$ & 70 & N & $\mathrm{N}$ & N & 150 & .16 \\
\hline WV0246R & $N$ & 5,000 & $N$ & 100 & $N$ & $<10$ & $N$ & 200 & .01 \\
\hline WW0247R & $\mathrm{N}$ & N & $\mathbb{N}$ & 150 & $\mathbf{N}$ & $N$ & $N$ & 50 & .011 \\
\hline WV0248R1 & $N$ & 500 & $\mathrm{~N}$ & $<10$ & $N$ & N & $\mathbf{N}$ & 20 & .028 \\
\hline WV0248R2 & $\mathbf{N}$ & 200 & $N$ & 15 & $N$ & $N$ & $N$ & 20 & 2.6 \\
\hline WV0249R & $N$ & 500 & $\mathbb{N}$ & 200 & $\mathbb{N}$ & 20 & N & 200 & .011 \\
\hline WV0250R & $N$ & 700 & $N$ & 500 & $N$ & 50 & $<200$ & 100 & .005 \\
\hline WV0251R & $N$ & 200 & $\mathbf{N}$ & 300 & $N$ & 30 & $<200$ & 70 & .002 \\
\hline WV0252RI & N & 500 & $N$ & 30 & $\mathbb{N}$ & N & $N$ & 50 & $<.001$ \\
\hline WV0252R2 & $N$ & N & $N$ & $<10$ & $N$ & N & N & $<10$ & .22 \\
\hline WV0253R & N & 500 & $N$ & 70 & $\mathbf{N}$ & N & N & 100 & $<.001$ \\
\hline WV0254R & $N$ & 300 & $\mathrm{~N}$ & 30 & $\mathbb{N}$ & N & N & 100 & .001 \\
\hline WV0255R & $\mathbb{N}$ & 300 & $\mathrm{~N}$ & 30 & $N$ & $N$ & $N$ & 100 & $<.001$ \\
\hline WV0256R & $\mathbb{N}$ & 300 & $\mathbb{N}$ & 30 & $\mathrm{~N}$ & $<10$ & $\mathrm{~N}$ & 100 & $<.001$ \\
\hline WV0257R & $\mathrm{N}$ & 500 & $N$ & 150 & $\mathrm{~N}$ & 15 & $\mathrm{~N}$ & 150 & .002 \\
\hline WV0258R & $N$ & 200 & $N$ & 150 & $\mathbf{N}$ & 15 & $<200$ & 150 & .003 \\
\hline
\end{tabular}


Table 1. Gold and spectrographic analyses of 110 outcrop samples.--Continued

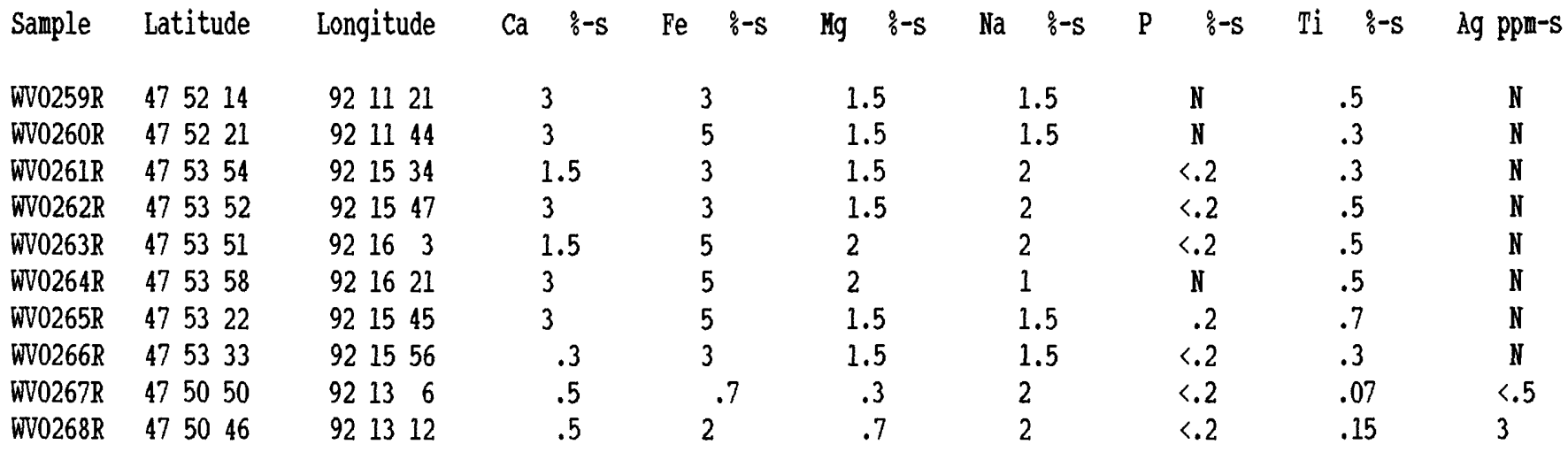


Table 1. Gold and spectrographic analyses of 110 outcrop samples.--Continued

\begin{tabular}{|c|c|c|c|c|c|c|c|c|c|}
\hline Sample & As $\mathrm{ppm}-\mathrm{s}$ & B ppm-s & Ba ppm-s & $\mathrm{Be} p p m-\mathrm{s}$ & Bi ppm-s & Cd ppm-s & Co ppm-s & Cr ppm-s & Cu ppm-s \\
\hline WV0259R & $\mathrm{N}$ & $\mathrm{N}$ & 70 & N & N & $\mathrm{N}$ & 30 & 300 & 50 \\
\hline WV0260R & N & $<10$ & 70 & $\mathrm{~N}$ & $\mathrm{~N}$ & $\mathrm{~N}$ & 30 & 200 & 50 \\
\hline WV0261R & $\mathrm{N}$ & $<10$ & 1,000 & 1.5 & $\mathrm{~N}$ & $\mathrm{~N}$ & 20 & 200 & 15 \\
\hline WV0262R & N & 15 & 700 & $<1$ & $\mathrm{~N}$ & $\mathrm{~N}$ & 15 & 100 & 30 \\
\hline WV0263R & $\mathrm{N}$ & 20 & 500 & $<1$ & $\mathrm{~N}$ & $\mathrm{~N}$ & 30 & 150 & 150 \\
\hline WV0264R & N & 10 & 50 & $<1$ & $\mathrm{~N}$ & $\mathrm{~N}$ & 50 & 500 & 50 \\
\hline WV265R & N & 20 & 700 & 1 & $\mathrm{~N}$ & N & 30 & 300 & 150 \\
\hline WV266R & $\mathrm{N}$ & 15 & 700 & $<1$ & N & $\mathrm{N}$ & 15 & 200 & 20 \\
\hline WO267R & N & 15 & 200 & $<1$ & $\mathrm{~N}$ & N & $\mathrm{N}$ & 15 & 15 \\
\hline WV268R & N & 50 & 700 & 1.5 & $\mathrm{~N}$ & $\mathrm{~N}$ & $<10$ & 15 & 7 \\
\hline
\end{tabular}


Table 1. Gold and spectrographic analyses of 110 outcrop samples.--Continued

Sample Ga ppm-s Ge ppm-s La ppm-s In ppm-s Mo ppm-s $\quad \mathbb{b}$ ppm-s Ni ppm-s pb ppm-s Sb ppm-s Sc ppm-s

$\begin{array}{lllrrrrrrrr}\text { WV0259R } & 30 & \text { N } & \text { N } & 1,500 & \text { N } & \text { N } & 100 & \text { N } & \text { N } & 30 \\ \text { WV0260R } & 30 & \text { N } & \text { N } & 1,500 & \text { N } & \text { N } & 70 & \text { N } & \text { N } & 30 \\ \text { WV0261R } & 30 & \text { N } & <50 & 700 & \text { N } & \text { N } & 70 & 15 & \text { N } & 10 \\ \text { WV0262R } & 50 & \text { N } & <50 & 1,000 & \text { N } & \text { N } & 15 & 15 & \text { N } & 10 \\ \text { WV0263R } & 50 & \text { N } & <50 & 1,000 & \text { N } & <20 & 50 & 10 & \text { N } & 15 \\ \text { WV0264R } & 50 & \text { N } & \text { N } & 1,000 & \text { N } & \text { N } & 70 & \text { N } & \text { N } & 30 \\ \text { WV0265R } & 50 & \text { N } & 70 & 1,000 & \text { N } & <20 & 70 & 30 & \text { N } & 20 \\ \text { WV0266R } & 30 & \text { N } & <50 & 700 & \text { N } & <20 & 70 & <10 & \text { N } & 10 \\ \text { WV0267R } & 20 & \text { N } & \text { N } & 100 & \text { N } & \text { N } & 5 & \text { N } & \text { N } & <5 \\ \text { WV0268R } & 50 & \text { N } & \text { N } & 500 & \text { N } & \text { N } & 15 & <10 & \text { N } & 5\end{array}$


Table 1. Gold and spectrographic analyses of 110 outcrop samples.--Continued

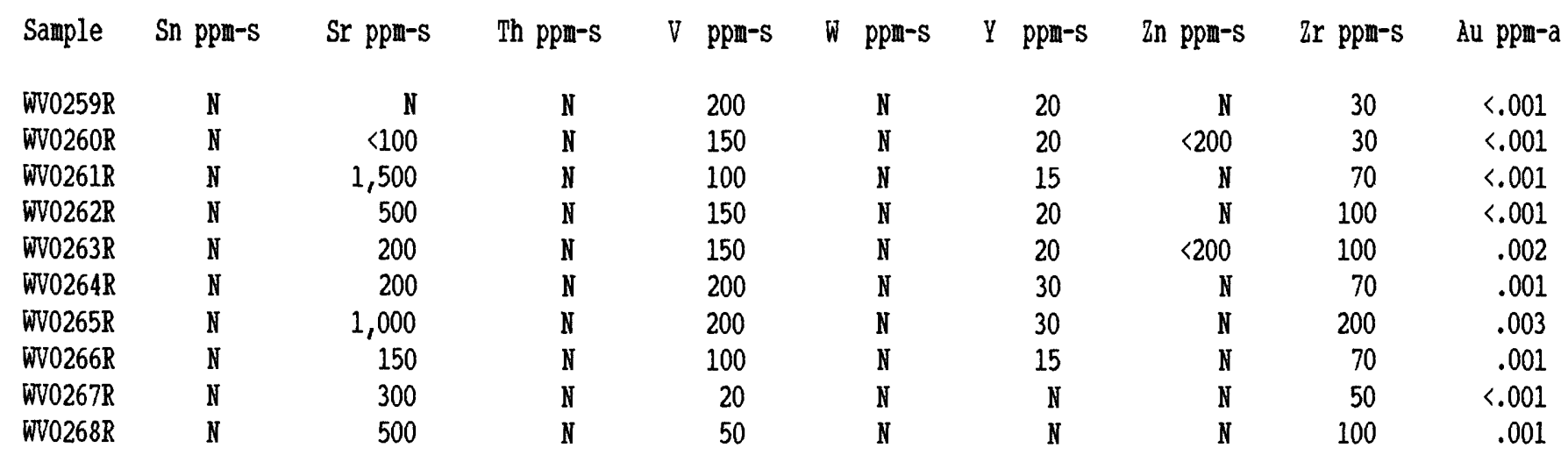


Table 2. Gold and spectrographic analyses of 238 B-horizon soil samples.

[N, not detected; <, detected but below the limit of determination showni >, determined to be greater than the value shown.]

\begin{tabular}{|c|c|c|c|c|c|c|c|c|c|}
\hline Sample & Latitude & Longitude & $\mathrm{Ca} \%-\mathrm{s}$ & $\mathrm{Fe}: \%-\mathrm{s}$ & $\mathrm{Hg} \quad \%-\mathrm{S}$ & $\mathrm{Na} \div-\mathrm{s}$ & $:-s$ & $\mathrm{Ti} \quad:-\mathrm{s}$ & $\mathrm{Ag} \mathrm{ppm-s}$ \\
\hline WN001 & $4751 \quad 10$ & $92 \quad 22 \quad 12$ & .7 & 5 & 1 & 2 & .3 & .7 & $<.5$ \\
\hline WV002 & 475456 & 921950 & .7 & 3 & .7 & 2 & .7 & .5 & .5 \\
\hline WV003 & 475458 & $92 \quad 21 \quad 8$ & .7 & 1.5 & .3 & 2 & $<.2$ & .5 & $<.5$ \\
\hline WV004 & 475440 & $92 \quad 22 \quad 2$ & .7 & 2 & .5 & 1.5 & $<.2$ & 1 & N \\
\hline WV005 & $4754 \quad 13$ & $92 \quad 2258$ & .7 & 3 & .7 & 2 & .2 & .7 & .5 \\
\hline WN006 & 475354 & $92 \quad 23 \quad 1$ & .7 & 5 & .7 & 1.5 & $<.2$ & .7 & N \\
\hline W007 & 475358 & $92 \quad 23 \quad 23$ & 1 & 3 & .7 & 2 & $<.2$ & .7 & N \\
\hline WV008 & 475429 & 922740 & .7 & 3 & .7 & 1.5 & $<.2$ & .5 & N \\
\hline WV009 & 475428 & 922725 & .7 & 3 & .7 & 1.5 & .3 & .5 & $<.5$ \\
\hline WV010 & $4754 \quad 25$ & $92 \quad 2657$ & .7 & 3 & .7 & 2 & $<.2$ & .5 & $N$ \\
\hline WV11 & $4754 \quad 5$ & $92 \quad 2648$ & .7 & 5 & .7 & 1.5 & .3 & .5 & $<.5$ \\
\hline WW012 & 475434 & 922552 & .7 & 3 & .3 & 1.5 & .3 & .5 & $<.5$ \\
\hline WV013 & $\begin{array}{lll}47 & 54 & 17\end{array}$ & 922536 & .7 & 3 & .7 & 1.5 & $<.2$ & .7 & $\mathrm{~N}$ \\
\hline WV014 & $4754 \quad 22$ & 92256 & .3 & 3 & .5 & 1.5 & $<.2$ & .5 & $\mathbb{N}$ \\
\hline WV015 & $4754 \quad 5$ & $92 \quad 2620$ & .5 & 3 & .7 & 1.5 & .7 & .5 & $<.5$ \\
\hline WV016 & 475331 & $92 \quad 2630$ & .7 & 3 & .5 & 1.5 & .2 & .7 & $<.5$ \\
\hline WV017 & $4753 \quad 46$ & $92 \quad 2552$ & .7 & 3 & .7 & 2 & .2 & .5 & $<.5$ \\
\hline WV018 & 475353 & $92 \quad 2518$ & .3 & 5 & .7 & 1 & .5 & .7 & $<.5$ \\
\hline WV019 & $4754 \quad 6$ & $92 \quad 25 \quad 5$ & .7 & 3 & .7 & 1.5 & $<.2$ & .7 & $N$ \\
\hline WV020 & 475355 & $92 \quad 24 \quad 48$ & 1 & 3 & .7 & 2 & $<.2$ & .7 & N \\
\hline WV021 & $4754 \quad 5$ & $92 \quad 24 \quad 19$ & .7 & 3 & .7 & 1.5 & $<.2$ & .7 & N \\
\hline WV022 & $47 \quad 54 \quad 12$ & 922350 & .7 & 3 & .7 & 1.5 & $<.2$ & .5 & N \\
\hline WV023 & $\begin{array}{lll}47 & 54 \quad 27\end{array}$ & $92 \quad 23 \quad 25$ & .7 & 3 & .7 & 1.5 & .2 & .5 & N \\
\hline WV024 & 475430 & 922250 & .7 & 3 & .7 & 2 & .2 & .5 & N \\
\hline WV025 & $4753 \quad 49$ & $92 \quad 23 \quad 27$ & 7 & 3 & 1 & 2 & N & .3 & N \\
\hline WV026 & 475326 & $9224 \quad 9$ & 1.5 & 3 & .7 & 1.5 & $<.2$ & .5 & $<.5$ \\
\hline WV027 & $4753 \quad 15$ & $92 \quad 24 \quad 22$ & .3 & 3 & .3 & 1.5 & .5 & .5 & 1 \\
\hline WV028 & $47 \quad 47 \quad 14$ & $92 \quad 23 \quad 42$ & 1 & 3 & .7 & 1.5 & .2 & .7 & .7 \\
\hline WV029 & 474738 & $92 \quad 23 \quad 42$ & .3 & 3 & .3 & 1.5 & .2 & .7 & $<.5$ \\
\hline WV030 & $\begin{array}{lll}47 \quad 48 & 10\end{array}$ & 922338 & .7 & 3 & 1 & 1.5 & $<.2$ & 1 & 1.5 \\
\hline WV031 & $\begin{array}{lll}47 & 48 & 18\end{array}$ & $92 \quad 2253$ & .7 & 3 & 1 & 2 & $<.2$ & .7 & $<.5$ \\
\hline WV032 & 474859 & $92 \quad 24 \quad 12$ & .5 & 3 & 1 & 2 & .2 & .5 & .5 \\
\hline WV033 & $4748 \quad 36$ & $92 \quad 24 \quad 2$ & .7 & 2 & .7 & 1.5 & $<.2$ & .7 & 1.5 \\
\hline W034 & $47 \quad 47 \quad 14$ & 922416 & .7 & 3 & 1 & 2 & .2 & .5 & $<.5$ \\
\hline WV035 & $\begin{array}{lll}47 & 47 & 6\end{array}$ & $92 \quad 24 \quad 48$ & .7 & 3 & .7 & 1.5 & $<.2$ & .7 & $N$ \\
\hline WV036 & $47 \quad 46 \quad 49$ & 922521 & 1.5 & 3 & .7 & 1.5 & $<.2$ & .5 & N \\
\hline WV037 & $4747 \quad 10$ & $92 \quad 2532$ & .7 & 3 & .7 & 1.5 & $<.2$ & .7 & .7 \\
\hline WV038 & $47 \quad 4639$ & 922630 & 1 & 3 & .7 & 2 & $<.2$ & .5 & N \\
\hline WV039 & $\begin{array}{lll}47 & 47 & 2\end{array}$ & $92 \quad 2645$ & .7 & 3 & .7 & 1.5 & $<.2$ & .7 & N \\
\hline WV040 & $\begin{array}{lll}47 & 47 & 31\end{array}$ & 922637 & .7 & 3 & .7 & 2 & $<.2$ & .7 & $<.5$ \\
\hline WV041 & $\begin{array}{lll}47 & 47 & 47\end{array}$ & $9227 \quad 6$ & .7 & 3 & .7 & 1.5 & .2 & .5 & $<.5$ \\
\hline WV042 & $\begin{array}{lll}47 & 48 & 1\end{array}$ & 922729 & .7 & 2 & .7 & 1.5 & $<.2$ & .5 & N \\
\hline WV044 & 474620 & 922655 & 1.5 & 3 & .7 & 1.5 & .2 & .5 & $\mathrm{~N}$ \\
\hline WV045 & $4746 \quad 18$ & 922740 & .7 & 3 & .7 & 1.5 & .2 & 1 & N \\
\hline WV046 & 474558 & 922745 & 2 & 3 & 1 & 1.5 & $<.2$ & .5 & $\mathrm{~N}$ \\
\hline WV047 & $47 \quad 45 \quad 35$ & 922740 & 1.5 & 2 & .7 & 1.5 & .2 & .3 & $<.5$ \\
\hline WV048 & $\begin{array}{lll}47 & 45 & 12\end{array}$ & 922758 & .7 & 3 & .7 & 1.5 & $<.2$ & .7 & $\mathbb{N}$ \\
\hline WV049 & $47 \quad 45 \quad 11$ & 922832 & .7 & 3 & .5 & 1.5 & .2 & .5 & N \\
\hline WV050 & $\begin{array}{lll}47 & 45 & 7\end{array}$ & $9229 \quad 4$ & .7 & 3 & .7 & 1.5 & $<.2$ & .5 & N \\
\hline WV051 & $47 \quad 4647$ & 922758 & .7 & 3 & .7 & 1.5 & .2 & .5 & N \\
\hline
\end{tabular}


Table 2. Gold and spectrographic analyses of 238 B-horizon soil samples.--Continued

Sample As ppm-s B ppm-s Ba ppm-s Be ppm-s Bi ppm-s Cd ppm-s Co ppm-s Cr ppm-s Cu ppm-s

\begin{tabular}{|c|c|c|c|c|c|c|c|c|c|}
\hline WV001 & $\mathbf{N}$ & 30 & 700 & 1 & N & N & 20 & 150 & 20 \\
\hline WVOO2 & $\mathbf{N}$ & 20 & 700 & 1.5 & $\mathbb{N}$ & N & 10 & 100 & 30 \\
\hline WV003 & $\mathrm{N}$ & 15 & 1,500 & 1 & N & N & $<10$ & 70 & 10 \\
\hline WVOO4 & $\mathbf{N}$ & 30 & 1,000 & 1 & $N$ & N & $<10$ & 100 & 15 \\
\hline WV005 & $\mathrm{N}$ & 30 & 1,000 & 1 & $\mathbf{N}$ & N & 15 & 150 & 20 \\
\hline WV006 & $\mathbf{N}$ & 30 & 700 & $<1$ & $N$ & $\mathbb{N}$ & 30 & 200 & 20 \\
\hline WV007 & $\mathbf{N}$ & 30 & 1,000 & 1 & $\mathbf{N}$ & $\mathbb{N}$ & 15 & 100 & 15 \\
\hline WV008 & $\mathbf{N}$ & 20 & 700 & 1 & $\mathbf{N}$ & N & 15 & 100 & 15 \\
\hline WV009 & N & 50 & 1,000 & 1 & $\mathbf{N}$ & N & 15 & 100 & 30 \\
\hline WV010 & $N$ & 30 & 1,000 & 1 & $N$ & $N$ & 15 & 100 & 15 \\
\hline WV011 & N & 30 & 700 & 1 & $\mathbf{N}$ & N & 20 & 150 & 30 \\
\hline WV012 & N & 30 & 500 & 1 & $\mathbb{N}$ & N & 15 & 150 & 30 \\
\hline WV013 & N & 30 & 700 & 1 & $\mathbf{N}$ & N & 15 & 150 & 20 \\
\hline WV014 & N & 50 & 500 & 1.5 & $\mathbb{N}$ & N & 15 & 150 & 20 \\
\hline WV015 & $\mathbf{N}$ & 50 & 700 & 1 & $N$ & N & $<10$ & 150 & 30 \\
\hline WV016 & $\mathbf{N}$ & 30 & 700 & 1 & $\mathrm{~N}$ & $\mathrm{H}$ & 15 & 100 & 20 \\
\hline WV017 & N & 30 & 700 & 1 & N & N & 15 & 150 & 30 \\
\hline WV018 & $\mathbf{N}$ & 50 & 700 & 1.5 & $\mathbf{N}$ & N & 20 & 150 & 50 \\
\hline WV019 & N & 30 & 1,000 & 1 & N & $\mathbb{N}$ & 20 & 150 & 15 \\
\hline WV020 & N & 30 & 700 & 1 & $\mathbb{N}$ & N & 20 & 150 & 20 \\
\hline WV021 & N & 30 & 700 & 1 & $\mathbb{N}$ & $N$ & 20 & 150 & 20 \\
\hline WV022 & 200 & 20 & 700 & 1.5 & $\mathrm{~N}$ & N & 10 & 100 & 20 \\
\hline WV023 & 200 & 15 & 1,000 & 1.5 & $\mathrm{~N}$ & N & $<10$ & 150 & 15 \\
\hline WV024 & 200 & 30 & 1,000 & 1.5 & N & N & 15 & 100 & 15 \\
\hline WV025 & 200 & 30 & 300 & $<1$ & $\mathbf{N}$ & $N$ & 15 & 200 & 50 \\
\hline WV026 & 200 & 30 & 700 & 1 & $N$ & $N$ & 15 & 100 & 30 \\
\hline WV027 & $<200$ & 15 & 700 & 1 & $\mathbf{N}$ & N & 10 & 150 & 30 \\
\hline WV028 & N & 50 & 700 & 1 & $\mathbf{N}$ & $N$ & 15 & 150 & 15 \\
\hline WV029 & N & 50 & 700 & 1 & $\mathbf{N}$ & N & $<10$ & 70 & 30 \\
\hline WV030 & $\mathbf{N}$ & 50 & 1,000 & 1 & $N$ & $N$ & 15 & 100 & 15 \\
\hline WV031 & $N$ & 50 & 700 & 1 & $N$ & $\mathrm{~N}$ & 10 & 100 & 15 \\
\hline WV032 & $\mathrm{N}$ & 30 & 500 & 1 & $N$ & N & 15 & 150 & 20 \\
\hline WV033 & N & 30 & 700 & 1 & $\mathbb{N}$ & N & 10 & 150 & 15 \\
\hline WV034 & N & 30 & 700 & 1 & N & N & 15 & 150 & 20 \\
\hline WV035 & N & 30 & 700 & 1 & $\mathbf{N}$ & N & N & 100 & 15 \\
\hline WV036 & $\mathbf{N}$ & 30 & 700 & 1.5 & $\mathbf{N}$ & N & $\mathrm{N}$ & 100 & 15 \\
\hline WV037 & $\mathrm{N}$ & 30 & 700 & 1 & $N$ & $N$ & 10 & 100 & 15 \\
\hline WV038 & $\mathbb{N}$ & 30 & 700 & 1 & $\mathbf{N}$ & N & 15 & 100 & 20 \\
\hline WV039 & N & 50 & 700 & 1 & $\mathbb{N}$ & N & 15 & 150 & 15 \\
\hline WV040 & $\mathbf{N}$ & 30 & 700 & 1 & $\mathbf{N}$ & N & 10 & 100 & 15 \\
\hline WV041 & N & 15 & 500 & 1 & N & N & 20 & 150 & 30 \\
\hline WV042 & N & 20 & 500 & 1 & $N$ & $N$ & 10 & 100 & 15 \\
\hline WV044 & N & 20 & 700 & 1 & $\mathbf{N}$ & N & 20 & 100 & 20 \\
\hline WV045 & $\mathbf{N}$ & 20 & 700 & $<1$ & $\mathrm{~N}$ & N & 15 & 150 & 20 \\
\hline WV046 & N & 15 & 700 & $<1$ & $\mathbf{N}$ & $\mathbf{N}$ & 20 & 100 & 15 \\
\hline WV047 & N & 15 & 500 & 1 & $\mathbf{N}$ & $\mathrm{N}$ & 10 & 70 & 15 \\
\hline WV048 & $N$ & 15 & 700 & 1 & $\mathbf{N}$ & N & 15 & 150 & 15 \\
\hline WV049 & $N$ & 30 & 700 & 1 & $N$ & $N$ & 15 & 100 & 15 \\
\hline WV050 & $\mathbf{N}$ & 30 & 700 & 1 & N & N & 20 & 150 & 15 \\
\hline WV051 & $\mathrm{N}$ & 20 & 500 & 1 & $\mathbb{N}$ & N & 20 & 150 & 15 \\
\hline
\end{tabular}


Table 2. Gold and spectrographic analyses of 238 B-horizon soil samples.--Continued

Sample Ga ppm-s Ge ppm-S La ppm-S Kn ppm-S Mo ppm-s Nb ppm-s Ni ppm-S Pb ppm-S Sb ppm-S Sc ppm-S

\begin{tabular}{|c|c|c|c|c|c|c|c|c|c|c|}
\hline WV001 & 50 & $N$ & $<50$ & 1,000 & N & $<20$ & 50 & 30 & $\mathrm{~N}$ & 7 \\
\hline WVOO2 & 50 & $N$ & 50 & 300 & $\mathrm{~N}$ & $<20$ & 30 & 50 & $N$ & 7 \\
\hline WV003 & 50 & N & $<50$ & 150 & 15 & $<20$ & 15 & 50 & $\mathrm{~N}$ & 5 \\
\hline WV004 & 30 & $\mathbb{N}$ & $<50$ & 200 & $<5$ & $<20$ & 20 & 50 & $N$ & 7 \\
\hline WV005 & 50 & $\mathrm{~N}$ & $<50$ & 300 & $<5$ & $<20$ & 30 & 50 & $N$ & 7 \\
\hline WV006 & 30 & $\mathbb{N}$ & $<50$ & 1,000 & N & $<20$ & 70 & 30 & $N$ & 10 \\
\hline WV007 & 50 & $N$ & 70 & 700 & N & $<20$ & 30 & 50 & $\mathrm{~N}$ & 7 \\
\hline WV008 & 50 & $\mathbb{N}$ & $<50$ & 300 & $<5$ & $<20$ & 30 & 50 & $\mathrm{~N}$ & 7 \\
\hline WV009 & 50 & $N$ & 50 & 500 & $\mathbf{N}$ & $<20$ & 30 & 50 & $\mathbf{N}$ & 7 \\
\hline WW010 & 50 & $N$ & $<50$ & 500 & $N$ & $<20$ & 30 & 50 & N & 7 \\
\hline WV011 & 50 & $\mathbb{N}$ & $<50$ & 500 & $<5$ & $<20$ & 50 & 50 & $\mathbf{N}$ & 7 \\
\hline WV012 & 50 & N & $<50$ & 300 & $N$ & $<20$ & 30 & 50 & $N$ & 7 \\
\hline WV013 & 50 & $N$ & $<50$ & 300 & N & $<20$ & 50 & 50 & N & 7 \\
\hline WV014 & 50 & $N$ & $<50$ & 300 & $N$ & $<20$ & 30 & 50 & $\mathrm{~N}$ & 7 \\
\hline WV015 & 50 & $\mathbb{N}$ & $<50$ & 700 & $\mathbf{N}$ & $<20$ & 30 & 50 & $\mathrm{~N}$ & 7 \\
\hline WV016 & 50 & $\mathbb{N}$ & $<50$ & 300 & $<5$ & $<20$ & 30 & 50 & $\mathrm{~N}$ & 7 \\
\hline WV017 & 50 & $N$ & $<50$ & 500 & $\mathrm{~N}$ & $<20$ & 30 & 50 & N & 7 \\
\hline WV018 & 50 & $\mathbb{N}$ & $<50$ & 300 & $N$ & $<20$ & 30 & 50 & $N$ & 7 \\
\hline WV019 & 50 & $\mathrm{~N}$ & $<50$ & 700 & $\mathbf{N}$ & $<20$ & 50 & 50 & $N$ & 7 \\
\hline WV020 & 50 & $N$ & $<50$ & 300 & $N$ & $<20$ & 50 & 50 & $N$ & 7 \\
\hline WV021 & 50 & $\mathrm{~N}$ & 50 & 300 & $\mathbf{N}$ & $<20$ & 30 & 50 & $\mathbb{N}$ & 7 \\
\hline WV022 & 50 & $\mathbb{N}$ & $<50$ & 300 & $\mathbb{N}$ & $<20$ & 30 & 30 & $\mathrm{~N}$ & 7 \\
\hline WV023 & 50 & $N$ & $<50$ & 200 & $<5$ & $<20$ & 50 & 30 & N & 7 \\
\hline WV024 & 50 & $\mathrm{~N}$ & $<50$ & 300 & $N$ & $<20$ & 30 & 30 & N & 7 \\
\hline WV025 & 30 & $\mathbb{N}$ & $<50$ & 1,000 & N & N & 50 & 20 & $\mathbb{N}$ & 10 \\
\hline WV026 & 30 & $\mathbb{N}$ & 50 & 700 & $N$ & $<20$ & 50 & 50 & $N$ & 7 \\
\hline WV027 & 30 & $\mathbb{N}$ & $<50$ & 300 & $\mathbf{N}$ & $<20$ & 50 & 100 & $\mathrm{~N}$ & 7 \\
\hline WV028 & 50 & $\mathbb{N}$ & 50 & 1,000 & $N$ & $<20$ & 50 & 50 & $\mathrm{~N}$ & 7 \\
\hline WV029 & 50 & $N$ & 50 & 300 & 5 & $<20$ & 30 & 50 & $N$ & 7 \\
\hline WV030 & 50 & $\mathbb{N}$ & 50 & 1,000 & $<5$ & 20 & 50 & 30 & $N$ & 10 \\
\hline WV031 & 50 & $\mathrm{~N}$ & $<50$ & 300 & $N$ & $<20$ & 50 & 20 & $N$ & 7 \\
\hline WV032 & 50 & $N$ & $<50$ & 300 & $<5$ & $<20$ & 50 & 30 & $N$ & 7 \\
\hline WV033 & 30 & N & 50 & 150 & $\mathbb{N}$ & $<20$ & 30 & 30 & N & 7 \\
\hline WV034 & 50 & $N$ & $<50$ & 500 & $\mathbf{N}$ & $<20$ & 50 & 30 & $N$ & 7 \\
\hline WV035 & 30 & $\mathrm{~N}$ & $<50$ & 500 & $<5$ & $<20$ & 50 & 30 & $\mathrm{~N}$ & 7 \\
\hline WV036 & 30 & $\mathrm{~N}$ & 50 & 700 & $\mathbf{N}$ & $<20$ & 30 & 30 & $\mathrm{~N}$ & 7 \\
\hline WV037 & 30 & $\mathbf{N}$ & 50 & 300 & $<5$ & $<20$ & 30 & 30 & $\mathrm{~N}$ & 7 \\
\hline WV038 & 50 & $N$ & $<50$ & 500 & $N$ & $<20$ & 50 & 30 & $\mathbf{N}$ & 7 \\
\hline WV039 & 50 & N & $<50$ & 500 & $N$ & $<20$ & 50 & 30 & $N$ & 10 \\
\hline WV040 & 50 & $N$ & $<50$ & 500 & $\mathbf{N}$ & $<20$ & 30 & 30 & $N$ & 7 \\
\hline WV041 & 30 & $N$ & $<50$ & 700 & N & $<20$ & 50 & 20 & $\mathrm{~N}$ & 7 \\
\hline WV042 & 30 & $\mathbb{N}$ & $<50$ & 500 & $\mathrm{~N}$ & $<20$ & 30 & 30 & $\mathrm{~N}$ & 7 \\
\hline WV044 & 50 & N & $<50$ & 1,000 & $\mathbb{N}$ & $<20$ & 50 & 30 & $\mathrm{~N}$ & 7 \\
\hline WV045 & 50 & $N$ & $<50$ & 500 & $<5$ & 20 & 30 & 30 & $\mathrm{~N}$ & 10 \\
\hline WV046 & 50 & $\mathbf{N}$ & $<50$ & 700 & $\mathrm{~N}$ & $<20$ & 50 & 30 & $\mathbf{N}$ & 7 \\
\hline WN047 & 30 & $N$ & $<50$ & 700 & N & $N$ & 30 & 20 & $\mathbf{N}$ & 7 \\
\hline WV048 & 30 & $\mathbf{N}$ & $<50$ & 500 & N & $<20$ & 30 & 30 & $\mathbf{N}$ & 7 \\
\hline WV049 & 30 & $\mathrm{~N}$ & $<50$ & 500 & N & $<20$ & 30 & 30 & $N$ & 7 \\
\hline WV050 & 30 & $\mathbf{N}$ & $<50$ & 500 & N & $<20$ & 50 & 30 & $\mathbf{N}$ & 7 \\
\hline WV051 & 50 & $N$ & $<50$ & 700 & N & $<20$ & 50 & 30 & $N$ & 7 \\
\hline
\end{tabular}


Table 2. Gold and spectrographic analyses of 238 B-horizon soil samples.--Continued

Sample Sn ppm-s Sr ppm-s Th ppm-s $\quad$ V ppm-s W ppm-s $\quad$ Y ppm-s $\quad$ Zn ppm-s $\quad$ Ir ppm-s Au ppm-a

\begin{tabular}{|c|c|c|c|c|c|c|c|c|c|}
\hline WV001 & $\mathbb{N}$ & 300 & $\mathrm{~N}$ & 150 & N & 15 & $<200$ & 200 & .01 \\
\hline WOO2 & $\mathbb{N}$ & 300 & $\mathbb{N}$ & 100 & $\mathrm{~N}$ & 15 & $\mathbf{N}$ & 150 & .001 \\
\hline WV003 & $\mathbf{N}$ & 700 & $\mathbb{N}$ & 50 & $\mathrm{~N}$ & 10 & $\mathbb{N}$ & 300 & $<.001$ \\
\hline WV004 & $\mathbf{N}$ & 300 & N & 100 & $\mathbb{N}$ & 20 & N & 700 & $<.001$ \\
\hline WV005 & $\mathbb{N}$ & 500 & $\mathrm{~N}$ & 100 & $\mathrm{~N}$ & 15 & $\mathbb{N}$ & 300 & $<.001$ \\
\hline WV006 & $N$ & 300 & $\mathrm{~N}$ & 150 & $\mathrm{~N}$ & 20 & $\mathbb{N}$ & 150 & .001 \\
\hline WV007 & $\mathrm{N}$ & 500 & $\mathrm{~N}$ & 150 & N & 20 & $\mathrm{~N}$ & 300 & .001 \\
\hline WV008 & $N$ & 300 & $N$ & 100 & $N$ & 15 & N & 300 & $<.001$ \\
\hline WV009 & $N$ & 500 & $\mathrm{~N}$ & 100 & $N$ & 20 & $<200$ & 300 & .002 \\
\hline WV010 & $N$ & 300 & $N$ & 100 & $\mathrm{~N}$ & 15 & N & 200 & .007 \\
\hline WV011 & $N$ & 300 & $\mathbf{N}$ & 150 & $\mathbf{N}$ & 15 & $<200$ & 200 & $<.001$ \\
\hline WV012 & $\mathbb{N}$ & 300 & $\mathbb{N}$ & 150 & $N$ & 15 & $\mathrm{~N}$ & 200 & $<.001$ \\
\hline WV013 & $N$ & 300 & $N$ & 150 & $N$ & 15 & $\mathrm{~N}$ & 300 & .001 \\
\hline WV014 & $N$ & 200 & $\mathbb{N}$ & 150 & N & 20 & $\mathrm{~N}$ & 200 & $<.001$ \\
\hline WV015 & $N$ & 200 & $\mathrm{~N}$ & 150 & $N$ & 15 & $<200$ & 200 & $<.001$ \\
\hline WV016 & $N$ & 300 & $\mathrm{~N}$ & 150 & $N$ & 15 & $\mathbb{N}$ & 300 & $<.001$ \\
\hline WV017 & $\mathrm{N}$ & 300 & $\mathrm{~N}$ & 150 & $\mathrm{~N}$ & 15 & $<200$ & 300 & $<.001$ \\
\hline WV018 & $\mathbb{N}$ & 200 & $\mathrm{~N}$ & 200 & N & 15 & $<200$ & 200 & .002 \\
\hline WV019 & $N$ & 300 & $N$ & 150 & $N$ & 15 & $<200$ & 300 & $<.001$ \\
\hline WV020 & $\mathbb{N}$ & 300 & $\mathbf{N}$ & 100 & N & 15 & $\mathrm{~N}$ & 300 & $<.001$ \\
\hline WV021 & $N$ & 300 & $N$ & 150 & N & 15 & N & 200 & $<.001$ \\
\hline WV022 & $N$ & 300 & $\mathrm{~N}$ & 150 & $N$ & 10 & $\mathrm{~N}$ & 300 & .009 \\
\hline WV023 & $N$ & 300 & $\mathbb{N}$ & 150 & $\mathbb{N}$ & 15 & $\mathrm{~N}$ & 200 & $<.001$ \\
\hline WV024 & $N$ & 500 & $\mathrm{~N}$ & 150 & $\mathbb{N}$ & 15 & $\mathrm{~N}$ & 200 & .001 \\
\hline WV025 & $N$ & 300 & $\mathrm{~N}$ & 150 & N & 20 & $\mathrm{~N}$ & 70 & .001 \\
\hline WV026 & $\mathrm{N}$ & 300 & $\mathbb{N}$ & 150 & $\mathbb{N}$ & 15 & $\mathrm{~N}$ & 150 & .002 \\
\hline WV027 & $\mathrm{N}$ & 150 & $N$ & 150 & $\mathbb{N}$ & 15 & $\mathrm{~N}$ & 100 & .002 \\
\hline WV028 & $\mathbb{N}$ & 300 & $N$ & 150 & $N$ & 15 & $<200$ & 300 & .002 \\
\hline WV029 & $N$ & 300 & $\mathrm{~N}$ & 150 & N & 20 & $<200$ & 150 & .002 \\
\hline WV030 & $\mathbf{N}$ & 300 & $N$ & 150 & $N$ & 20 & 200 & 300 & .001 \\
\hline WV031 & N & 300 & $N$ & 150 & $N$ & 15 & $\mathbf{N}$ & 150 & .002 \\
\hline WV032 & $\mathbb{N}$ & 300 & $N$ & 150 & $N$ & 15 & $\mathrm{~N}$ & 100 & .005 \\
\hline WV033 & $\mathrm{N}$ & 500 & $\mathrm{~N}$ & 100 & $N$ & 15 & $\mathrm{~N}$ & 200 & .008 \\
\hline WV034 & $\mathbf{N}$ & 300 & $N$ & 150 & $N$ & 15 & $\mathrm{~N}$ & 150 & .006 \\
\hline WV035 & $\mathbf{N}$ & 500 & $N$ & 100 & N & 15 & $\mathbb{N}$ & 300 & .001 \\
\hline WV036 & $\mathbf{N}$ & 500 & $N$ & 150 & $N$ & 15 & $\mathbb{N}$ & 300 & $<.001$ \\
\hline WV037 & $\mathbb{N}$ & 300 & $\mathrm{~N}$ & 150 & $\mathbb{N}$ & 15 & $\mathbb{N}$ & 300 & .026 \\
\hline WV038 & $\mathrm{N}$ & 300 & N & 150 & N & 15 & $\mathbb{N}$ & 70 & $<.001$ \\
\hline WV039 & $N$ & 300 & $N$ & 150 & N & 15 & $\mathbf{N}$ & 200 & .001 \\
\hline WV040 & N & 300 & $N$ & 150 & N & 15 & $\mathrm{~N}$ & 300 & .001 \\
\hline WV041 & $N$ & 300 & $\mathbb{N}$ & 150 & $N$ & 15 & $<200$ & 200 & .001 \\
\hline WV042 & $N$ & 300 & $N$ & 100 & $N$ & 15 & $\mathrm{~N}$ & 300 & .001 \\
\hline WV044 & $N$ & 500 & $\mathrm{~N}$ & 100 & $\mathbf{N}$ & 15 & $<200$ & 100 & $<.001$ \\
\hline WV045 & $N$ & 300 & $N$ & 150 & $N$ & 15 & $\mathbb{N}$ & 300 & .003 \\
\hline WV046 & $N$ & 300 & $\mathbf{N}$ & 100 & $\mathbf{N}$ & 10 & $<200$ & 150 & .001 \\
\hline WV047 & $N$ & 300 & $N$ & 70 & $\mathbf{N}$ & 10 & $N$ & 70 & $<.001$ \\
\hline WV048 & $N$ & 300 & N & 100 & N & 10 & N & 150 & $<.001$ \\
\hline WV049 & $N$ & 500 & $\mathbf{N}$ & 100 & $\mathbf{N}$ & 15 & $\mathbf{N}$ & 200 & $<.001$ \\
\hline WV050 & $\mathbf{N}$ & 300 & $\mathrm{~N}$ & 100 & $N$ & 15 & $<200$ & 300 & $<.001$ \\
\hline WV051 & $N$ & 300 & $\mathbb{N}$ & 150 & N & 15 & $<200$ & 300 & .001 \\
\hline
\end{tabular}


Table 2. Gold and spectrographic analyses of 238 B-horizon soil samples.--Continued

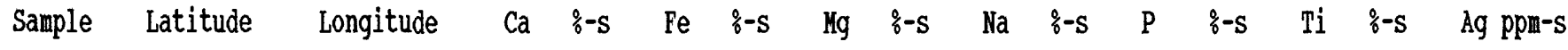

\begin{tabular}{|c|c|c|c|c|c|c|c|c|c|}
\hline WV053 & $4746 \quad 24$ & 922836 & 1.5 & 2 & .7 & 1.5 & $<.2$ & .5 & $\mathrm{~N}$ \\
\hline WV054 & $4746 \quad 15$ & 922914 & .7 & 3 & .7 & 1.5 & .2 & .5 & $\mathbb{N}$ \\
\hline WV055 & $4746 \quad 8$ & 92301 & .5 & 3 & .7 & 1.5 & .2 & .5 & $<.5$ \\
\hline WV056 & 474635 & 92303 & .7 & 3 & .7 & 1.5 & $<.2$ & .5 & $\mathbb{N}$ \\
\hline WV057 & 474659 & 923011 & .7 & 3 & .7 & 1.5 & $<.2$ & .5 & $<.5$ \\
\hline WV058 & $\begin{array}{lll}47 & 47 & 27\end{array}$ & $9230 \quad 7$ & .7 & 3 & .7 & 1.5 & $<.2$ & .3 & $N$ \\
\hline WV059 & $4747 \quad 44$ & 923010 & .5 & 3 & .7 & 1.5 & $<.2$ & .3 & $<.5$ \\
\hline WV060 & $47 \quad 49 \quad 12$ & 923010 & .7 & 3 & .7 & 1.5 & $<.2$ & .5 & N \\
\hline WV061 & $47 \quad 4934$ & 923014 & 1 & 3 & .7 & 1.5 & $<.2$ & .5 & $\mathbb{N}$ \\
\hline WV062 & 474951 & $9230 \quad 40$ & .7 & 3 & .7 & 1.5 & $<.2$ & .5 & $N$ \\
\hline WV063 & $47 \quad 5011$ & $9231 \quad 15$ & .7 & 3 & .7 & 1.5 & $<.2$ & .7 & $\mathbb{N}$ \\
\hline WV064 & $47 \quad 47 \quad 24$ & 922929 & .7 & 3 & .7 & 1.5 & $<.2$ & .7 & $<.5$ \\
\hline WV065 & $47 \quad 46 \quad 19$ & 923033 & 1.5 & 3 & .7 & 1.5 & .2 & .5 & $\mathbb{N}$ \\
\hline WV066 & 474556 & $9230 \quad 40$ & .7 & 3 & .5 & 1.5 & $<.2$ & .5 & $<.5$ \\
\hline W067 & 474625 & 923114 & .5 & 3 & .5 & 1.5 & $<.2$ & .5 & $<.5$ \\
\hline WV068 & $\begin{array}{lll}47 & 46 & 27\end{array}$ & 9231.45 & .7 & 3 & .7 & 1.5 & $<.2$ & .3 & $N$ \\
\hline WV069 & 474650 & 923131 & .5 & 3 & .7 & 1.5 & $<.2$ & .7 & $<.5$ \\
\hline WV070 & 474721 & $9231 \quad 17$ & .3 & 2 & .5 & 1.5 & $<.2$ & .5 & $N$ \\
\hline WV071 & 474738 & 923138 & .5 & 2 & .7 & 1.5 & $<.2$ & .3 & .7 \\
\hline WV072 & 474634 & 923218 & .3 & 1.5 & .3 & 1.5 & $<.2$ & .3 & .7 \\
\hline WV073 & $4746 \quad 46$ & 923251 & .3 & 3 & 1 & 1.5 & $<.2$ & .5 & $<.5$ \\
\hline WV074 & 474655 & 923328 & .3 & 3 & .7 & 1.5 & $<.2$ & .5 & .5 \\
\hline WV075 & 474714 & 923258 & .5 & 2 & .7 & 2 & $<.2$ & .5 & $\mathbb{N}$ \\
\hline WV076 & 474656 & $9234 \quad 2$ & .3 & 2 & .5 & 1.5 & $<.2$ & .5 & .5 \\
\hline WV077 & 474626 & 923356 & .3 & 3 & .7 & 1.5 & $<.2$ & .7 & $<.5$ \\
\hline WV078 & $4746 \quad 4$ & 923348 & .5 & 3 & .7 & 1.5 & $<.2$ & .5 & $\mathrm{~N}$ \\
\hline WV079 & $47 \quad 45 \quad 42$ & 923359 & .3 & 3 & .7 & 1.5 & $<.2$ & .7 & $<.5$ \\
\hline WV080 & $4747 \quad 22$ & 923358 & .3 & 1.5 & .5 & 1.5 & $<.2$ & .3 & N \\
\hline WV081 & $\begin{array}{lll}47 & 48 & 8\end{array}$ & $9234 \quad 1$ & .3 & 3 & .5 & 1.5 & $<.2$ & .3 & N \\
\hline WV082 & $47 \quad 48 \quad 15$ & 923326 & .5 & 2 & .5 & 1.5 & $<.2$ & .5 & $\mathbb{N}$ \\
\hline WV083 & 475019 & 923252 & .7 & 1.5 & .7 & 1.5 & $<.2$ & .5 & $N$ \\
\hline WV084 & 474952 & 923249 & .5 & 2 & .7 & 2 & $<.2$ & .5 & $\mathbb{N}$ \\
\hline WV085 & 474954 & 92329 & .7 & 3 & .7 & 2 & $<.2$ & .7 & $\mathbb{N}$ \\
\hline WV086 & 474952 & 923135 & .7 & 3 & .7 & 1.5 & $<.2$ & .7 & $\mathbb{N}$ \\
\hline WV087 & 474955 & 923331 & .5 & 2 & .7 & 1.5 & $<.2$ & .3 & $\mathbb{N}$ \\
\hline WV088 & 474956 & $92 \quad 34 \quad 17$ & .5 & 3 & .7 & 1.5 & $<.2$ & .7 & $<.5$ \\
\hline WV089 & 474955 & 923519 & .5 & 3 & 1 & 1.5 & $<.2$ & .7 & $<.5$ \\
\hline WV090 & $\begin{array}{lll}47 & 50 & 27\end{array}$ & 923519 & .5 & 3 & 1 & 1.5 & $<.2$ & .5 & N \\
\hline WV091 & 474916 & 923514 & .7 & 2 & .7 & 1.5 & $<.2$ & .5 & N \\
\hline WV092 & 474838 & 923518 & .3 & 3 & .7 & 1.5 & $<.2$ & .5 & $<.5$ \\
\hline WV093 & $47 \quad 48 \quad 11$ & 923518 & .3 & 3 & .7 & 2 & $<.2$ & .5 & $\mathrm{~N}$ \\
\hline WV094 & 474735 & 923514 & .5 & 1.5 & .5 & 2 & $<.2$ & .3 & $\mathbb{N}$ \\
\hline WV095 & $\begin{array}{lll}47 & 48 & 8\end{array}$ & 923614 & .3 & 3 & .7 & 2 & $<.2$ & .5 & N \\
\hline WV096 & $47 \quad 47 \quad 44$ & 923616 & .3 & 3 & .7 & 1.5 & $<.2$ & .5 & $\mathrm{~N}$ \\
\hline WV097 & 474716 & 923620 & .5 & 2 & .7 & 1.5 & $<.2$ & .7 & $\mathbb{N}$ \\
\hline WV098 & 474651 & 923635 & .3 & 3 & .5 & 1.5 & .3 & .5 & $N$ \\
\hline WVO99 & 474652 & 923551 & .5 & 2 & .7 & 1.5 & $<.2$ & .5 & $\mathrm{~N}$ \\
\hline WV100 & $\begin{array}{lll}47 & 47 & 8\end{array}$ & 922148 & .5 & 3 & .7 & 1.5 & $<.2$ & .7 & $<.5$ \\
\hline WV101 & 474710 & 922233 & .7 & 3 & .7 & 2 & $<.2$ & .5 & $\mathrm{~N}$ \\
\hline WV102 & 475039 & $92 \quad 2245$ & .5 & 3 & .5 & 1.5 & .2 & .3 & $<.5$ \\
\hline
\end{tabular}


Table 2. Gold and spectrographic analyses of 238 B-horizon soil samples.--Continued

Sample As ppm-s B ppm-s Ba ppm-s Be ppm-s Bi ppm-s Cd ppm-s Co ppm-s Cr ppm-s Cu ppm-s

\begin{tabular}{|c|c|c|c|c|c|c|c|c|c|}
\hline WV053 & N & 15 & 500 & 1 & N & $N$ & 15 & 70 & 15 \\
\hline WV054 & $\mathrm{N}$ & 20 & 700 & 1 & N & N & 20 & 150 & 15 \\
\hline WV055 & $\mathrm{N}$ & 20 & 700 & 1 & $\mathrm{~N}$ & N & 10 & 150 & 30 \\
\hline WV056 & $\mathrm{N}$ & 20 & 700 & 1 & N & N & 15 & 150 & 15 \\
\hline WV057 & N & 30 & 700 & 1 & N & N & 20 & 150 & 30 \\
\hline WV058 & N & 20 & 700 & 1 & N & N & 15 & 200 & 15 \\
\hline WV059 & N & 30 & 700 & $<1$ & N & $\mathbb{N}$ & 10 & 100 & 20 \\
\hline WV060 & N & 20 & 700 & 1 & N & N & 20 & 100 & 20 \\
\hline WV061 & $\mathrm{N}$ & 30 & 500 & 1 & N & $\mathbb{N}$ & 10 & 100 & 10 \\
\hline WV062 & $\mathbb{N}$ & 20 & 700 & 1 & $\mathbf{N}$ & N & 20 & 100 & 20 \\
\hline WV063 & N & 30 & 700 & 1 & N & N & 15 & 100 & 15 \\
\hline WV064 & $\mathbb{N}$ & 30 & 700 & 1 & N & N & 20 & 100 & 15 \\
\hline WV065 & N & 15 & 500 & $<1$ & $\mathbb{N}$ & N & 20 & 100 & 15 \\
\hline WV066 & N & 20 & 700 & 1 & N & N & 20 & 100 & 15 \\
\hline WV067 & N & 20 & 700 & $<1$ & $\mathbb{N}$ & $\mathbb{N}$ & 15 & 100 & 15 \\
\hline WV068 & $\mathrm{N}$ & 20 & 500 & 1 & N & $\mathbf{N}$ & 20 & 150 & 20 \\
\hline WV069 & N & 30 & 700 & 1 & $\mathrm{~N}$ & $N$ & 15 & 150 & 15 \\
\hline WV070 & $\mathrm{N}$ & 15 & 700 & 1 & $\mathrm{~N}$ & $\mathbf{N}$ & 15 & 70 & 15 \\
\hline WV071 & N & 20 & 500 & 1 & $\mathbb{N}$ & $\mathrm{N}$ & 15 & 100 & 15 \\
\hline WV072 & $\mathbb{N}$ & 30 & 700 & 1 & $N$ & $\mathrm{~N}$ & 10 & 70 & 15 \\
\hline WV073 & N & 30 & 500 & 1 & $\mathbb{N}$ & $\mathrm{N}$ & 30 & 150 & 30 \\
\hline WV074 & $\mathrm{N}$ & 30 & 700 & 1 & $N$ & $\mathbf{N}$ & 20 & 70 & 15 \\
\hline WV075 & N & 20 & 1,000 & $<1$ & $\mathbb{N}$ & $\mathbb{N}$ & 15 & 150 & 10 \\
\hline WV076 & N & 30 & 700 & $<1$ & $N$ & N & 10 & 50 & 15 \\
\hline WV077 & $\mathbb{N}$ & 20 & 1,000 & $<1$ & $\mathbb{N}$ & $\mathbb{N}$ & 15 & 150 & 15 \\
\hline WV078 & N & 30 & 700 & $<1$ & N & N & 20 & 100 & 15 \\
\hline WV079 & $\mathbf{N}$ & 20 & 1,000 & $<1$ & N & N & 20 & 150 & 15 \\
\hline WV080 & N & 30 & 700 & $<1$ & $N$ & N & $<10$ & 100 & 10 \\
\hline WV081 & N & 30 & 500 & 1 & N & N & 15 & 100 & 20 \\
\hline WV082 & N & 30 & 500 & 1 & N & N & 10 & 100 & 15 \\
\hline WV083 & N & 15 & 500 & 1 & $N$ & $\mathrm{~N}$ & $<10$ & 70 & 10 \\
\hline WV084 & N & 20 & 700 & $<1$ & $\mathbb{N}$ & $\mathbb{N}$ & 15 & 150 & 15 \\
\hline WV085 & $\mathbb{N}$ & 20 & 700 & 1 & $\mathrm{~N}$ & $\mathrm{~N}$ & 20 & 150 & 15 \\
\hline WV086 & N & 30 & 700 & 1.5 & $N$ & $\mathrm{~N}$ & 15 & 100 & 20 \\
\hline WV087 & $\mathbb{N}$ & 30 & 500 & 1.5 & $\mathrm{~N}$ & $\mathrm{~N}$ & 15 & 100 & 15 \\
\hline WV088 & $\mathbf{N}$ & 30 & 700 & 1 & $\mathbb{N}$ & N & 20 & 150 & 20 \\
\hline WV089 & N & 30 & 1,000 & 1.5 & $N$ & N & 20 & 200 & 20 \\
\hline WV090 & N & 50 & 700 & 1.5 & N & N & 30 & 150 & 20 \\
\hline WV091 & N & 20 & 700 & 1 & N & $\mathbb{N}$ & 15 & 150 & 15 \\
\hline WV092 & N & 30 & 700 & 1 & $\mathbb{N}$ & $\mathrm{N}$ & 15 & 150 & 20 \\
\hline WV093 & $\mathbb{N}$ & 50 & 700 & 1.5 & $\mathrm{~N}$ & N & 15 & 100 & 20 \\
\hline WV094 & $\mathbf{N}$ & 30 & 1,000 & $<1$ & $\mathbb{N}$ & $\mathrm{N}$ & $<10$ & 70 & 10 \\
\hline WW095 & N & 50 & 1,000 & 1 & N & $\mathrm{N}$ & 20 & 150 & 20 \\
\hline WV096 & N & 50 & 700 & 1 & N & $\mathrm{N}$ & 30 & 150 & 20 \\
\hline WV097 & $\mathbb{N}$ & 30 & 700 & $<1$ & $N$ & N & 15 & 70 & 15 \\
\hline WV098 & N & 30 & 700 & $<1$ & N & $\mathrm{N}$ & 15 & 70 & 15 \\
\hline WVO99 & $\mathbf{N}$ & 30 & 1,000 & 1 & N & N & 15 & 100 & 15 \\
\hline WV100 & N & 30 & 700 & 1 & $N$ & N & 20 & 100 & 20 \\
\hline WV101 & N & 30 & 700 & 1 & $N$ & N & 20 & 150 & 30 \\
\hline WV102 & N & 15 & 500 & 1 & N & $\mathrm{N}$ & 20 & 70 & 20 \\
\hline
\end{tabular}


Table 2. Gold and spectrographic analyses of 238 B-horizon soil samples.--Continued

Sample Ga ppm-s Ge ppm-s La ppm-s In ppm-s Ho ppm-s Mb ppm-s Ni ppm-s Pb ppm-s Sb ppm-s Sc ppm-s

\begin{tabular}{|c|c|c|c|c|c|c|c|c|c|c|}
\hline WV053 & 30 & N & $<50$ & 700 & $\mathrm{~N}$ & $<20$ & 30 & 20 & $\mathrm{~N}$ & 5 \\
\hline WV054 & 50 & N & $<50$ & 700 & $<5$ & $<20$ & 50 & 30 & $\mathbf{N}$ & 7 \\
\hline WN055 & 50 & N & $<50$ & 500 & N & $<20$ & 30 & 30 & $\mathrm{~N}$ & 7 \\
\hline WV056 & 50 & N & $<50$ & 700 & N & $<20$ & 50 & 30 & N & 7 \\
\hline WV057 & 50 & N & 50 & 700 & N & $<20$ & 50 & 50 & N & 7 \\
\hline WV058 & 50 & N & $<50$ & 500 & N & $<20$ & 50 & 30 & N & 7 \\
\hline WV059 & 50 & N & $<50$ & 300 & N & $<20$ & 30 & 30 & N & 7 \\
\hline WV060 & 30 & N & $<50$ & 700 & $\mathbf{N}$ & $<20$ & 50 & 30 & N & 7 \\
\hline WV061 & 50 & N & $<50$ & 300 & N & $<20$ & 30 & 30 & $\mathbf{N}$ & 7 \\
\hline WV062 & 50 & $\mathbb{N}$ & $<50$ & 700 & N & $<20$ & 30 & 30 & $\mathbf{N}$ & 7 \\
\hline WN063 & 30 & N & $<50$ & 700 & N & $<20$ & 30 & 30 & N & 7 \\
\hline WV064 & 50 & N & 50 & 300 & N & $<20$ & 30 & 30 & N & 7 \\
\hline WV065 & 30 & N & $<50$ & 500 & N & $<20$ & 50 & 15 & N & 7 \\
\hline WV066 & 30 & N & $<50$ & 300 & N & $<20$ & 30 & 30 & $\mathbf{N}$ & 7 \\
\hline WV067 & 30 & N & $<50$ & 300 & N & $<20$ & 30 & 30 & $\mathbf{N}$ & 7 \\
\hline WV068 & 50 & N & $<50$ & 200 & $<5$ & $<20$ & 50 & 30 & N & 7 \\
\hline WV069 & 30 & N & $<50$ & 300 & N & $<20$ & 50 & 30 & N & 7 \\
\hline WV070 & 30 & N & $<50$ & 150 & N & $<20$ & 30 & 30 & $\mathbf{N}$ & 5 \\
\hline WV071 & 30 & $N$ & $<50$ & 150 & N & $<20$ & 30 & 30 & $\mathrm{~N}$ & 7 \\
\hline WV072 & 30 & $\mathbf{N}$ & 50 & 700 & $\mathbf{N}$ & $<20$ & 20 & 30 & $\mathbf{N}$ & 5 \\
\hline WN073 & 30 & N & $<50$ & 500 & N & $<20$ & 70 & 50 & N & 10 \\
\hline WN074 & 30 & N & $<50$ & 200 & N & $<20$ & 50 & 30 & $\mathbf{N}$ & 7 \\
\hline WV075 & 30 & N & $<50$ & 300 & N & $<20$ & 30 & 30 & N & 7 \\
\hline WV076 & 30 & N & $<50$ & 200 & N & $<20$ & 20 & 30 & $\mathbf{N}$ & 7 \\
\hline WV077 & 30 & N & 50 & 300 & N & 20 & 50 & 30 & N & 7 \\
\hline WV078 & 50 & N & $<50$ & 300 & N & $<20$ & 50 & 30 & $\mathbf{N}$ & 10 \\
\hline WV079 & 50 & N & $<50$ & 300 & N & $<20$ & 50 & 30 & $\mathrm{~N}$ & 7 \\
\hline WV080 & 30 & N & $<50$ & 200 & N & $<20$ & 30 & 30 & $\mathrm{~N}$ & 7 \\
\hline WV081 & 50 & N & $<50$ & 300 & N & $<20$ & 30 & 30 & $\mathrm{~N}$ & 10 \\
\hline WV082 & 50 & $\mathbf{N}$ & $<50$ & 300 & N & $<20$ & 30 & 30 & $\mathbf{N}$ & 7 \\
\hline WV083 & 20 & N & $<50$ & 200 & N & $<20$ & 20 & 20 & $\mathbb{N}$ & 7 \\
\hline WV084 & 30 & N & $<50$ & 200 & $N$ & $<20$ & 30 & 30 & N & 10 \\
\hline WV085 & 50 & N & $<50$ & 500 & $<5$ & $<20$ & 50 & 30 & N & 10 \\
\hline WV086 & 50 & N & $<50$ & 700 & N & $<20$ & 30 & 30 & $\mathbf{N}$ & 10 \\
\hline WV087 & 30 & N & $<50$ & 500 & N & $<20$ & 20 & 30 & $\mathbf{N}$ & 7 \\
\hline WV088 & 50 & N & $<50$ & 1,000 & N & $<20$ & 30 & 30 & $\mathbf{N}$ & 10 \\
\hline WV089 & 50 & N & 50 & 700 & N & $<20$ & 50 & 30 & $\mathrm{~N}$ & 10 \\
\hline WV090 & 50 & N & 50 & 1,000 & N & $<20$ & 50 & 30 & $\mathbf{N}$ & 10 \\
\hline Wv091 & 50 & N & $<50$ & 300 & N & $<20$ & 50 & 30 & $\mathrm{~N}$ & 7 \\
\hline WV092 & 50 & N & $<50$ & 300 & N & $<20$ & 50 & 30 & $\mathbb{N}$ & 7 \\
\hline Wv093 & 30 & N & $<50$ & 500 & $\mathbf{N}$ & $<20$ & 30 & 30 & $\mathbf{N}$ & 10 \\
\hline WV094 & 30 & N & $<50$ & 300 & N & $<20$ & 20 & 30 & $\mathbf{N}$ & 5 \\
\hline WV095 & 50 & N & 50 & 700 & N & $<20$ & 30 & 30 & $\mathbf{N}$ & 10 \\
\hline Wv096 & 50 & N & $<50$ & 300 & N & $<20$ & 50 & 30 & $\mathbf{N}$ & 10 \\
\hline WV097 & 30 & N & $<50$ & 500 & N & $<20$ & 30 & 30 & N & 7 \\
\hline Wv098 & 50 & N & $<50$ & 300 & 5 & $<20$ & 30 & 30 & $N$ & 7 \\
\hline Wvog9 & 30 & N & $<50$ & 300 & $\mathbf{N}$ & $<20$ & 30 & 30 & $\mathbf{N}$ & 7 \\
\hline WV100 & 30 & N & $<50$ & 500 & N & $<20$ & 50 & 30 & $\mathbf{N}$ & 7 \\
\hline WV101 & 50 & N & $<50$ & 700 & N & $<20$ & 50 & 150 & $\mathbf{N}$ & 7 \\
\hline WV102 & 30 & N & $<50$ & 700 & N & $<20$ & 30 & 30 & $\mathbb{N}$ & 7 \\
\hline
\end{tabular}


Table 2. Gold and spectrographic analyses of 238 B-horizon soil samples.--Continued

Sample Sn ppm-s Sr ppm-s Th ppm-s V ppm-s W ppm-s Y ppm-s in ppm-s lr ppm-s Au ppm-a

\begin{tabular}{|c|c|c|c|c|c|c|c|c|c|}
\hline WV053 & N & 300 & N & 70 & $\mathbb{N}$ & 10 & N & 70 & $<.001$ \\
\hline WV054 & $\mathbf{N}$ & 500 & $\mathbb{N}$ & 150 & N & 15 & $<200$ & 200 & .016 \\
\hline WV055 & $\mathbb{N}$ & 300 & N & 150 & $\mathbb{N}$ & 15 & N & 200 & .001 \\
\hline WV056 & $\mathbb{N}$ & 300 & N & 150 & $\mathbf{N}$ & 15 & $\mathbb{N}$ & 200 & .001 \\
\hline WV057 & $\mathbf{N}$ & 300 & $\mathbb{N}$ & 150 & N & 15 & $\mathbb{N}$ & 150 & .001 \\
\hline WV058 & $\mathbf{N}$ & 300 & N & 100 & 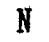 & 10 & N & 150 & .002 \\
\hline WV059 & N & 500 & $\mathbb{N}$ & 100 & N & 10 & $\mathbb{N}$ & 150 & .001 \\
\hline WV060 & $\mathbb{N}$ & 500 & N & 100 & $\mathrm{~N}$ & 15 & N & 100 & .001 \\
\hline WV061 & $\mathrm{N}$ & 500 & $\mathbb{N}$ & 100 & $\mathrm{~N}$ & 15 & $N$ & 150 & .002 \\
\hline WN062 & $\mathbf{N}$ & 300 & N & 100 & $\mathbf{N}$ & 15 & $N$ & 150 & $<.001$ \\
\hline WV063 & $\mathbf{N}$ & 300 & $\mathbb{N}$ & 100 & N & 15 & N & 150 & .002 \\
\hline WV064 & $\mathbb{N}$ & 500 & N & 100 & $\mathbf{N}$ & 15 & $\mathbb{N}$ & 300 & $<.001$ \\
\hline WV065 & $\mathbf{N}$ & 300 & $\mathbb{N}$ & 100 & $\mathbf{N}$ & 10 & N & 70 & $<.001$ \\
\hline WV066 & $\mathbb{N}$ & 300 & $\mathbb{N}$ & 100 & $\mathbb{N}$ & 15 & N & 150 & .001 \\
\hline WV067 & $\mathbf{N}$ & 300 & N & 150 & $\mathbb{N}$ & 20 & N & 200 & .001 \\
\hline WV068 & $N$ & 300 & N & 150 & $\mathbb{N}$ & 15 & N & 150 & $<.001$ \\
\hline WV069 & $\mathrm{N}$ & 300 & $\mathbb{N}$ & 100 & $\mathrm{~N}$ & 20 & $N$ & 300 & $<.001$ \\
\hline WV070 & $\mathbb{N}$ & 300 & N & 100 & $\mathbb{N}$ & 10 & $\mathrm{~N}$ & 200 & $<.001$ \\
\hline WV071 & $\mathrm{N}$ & 300 & N & 100 & $\mathrm{~N}$ & 15 & N & 150 & $<.001$ \\
\hline WV072 & $\mathbf{N}$ & 300 & N & 70 & $\mathrm{~N}$ & 15 & N & $200^{\circ}$ & $<.001$ \\
\hline WV073 & $\mathbf{N}$ & 300 & $\mathbb{N}$ & 150 & $\mathrm{~N}$ & 15 & $<200$ & 200 & .002 \\
\hline WV074 & $\mathbb{N}$ & 300 & N & 100 & $\mathbf{N}$ & 15 & N & 200 & .001 \\
\hline WV075 & $\mathbf{N}$ & 300 & $\mathbb{N}$ & 100 & $\mathbf{N}$ & 15 & $N$ & 300 & $<.001$ \\
\hline WV076 & $\mathbb{N}$ & 300 & N & 70 & $\mathrm{~N}$ & 15 & N & 300 & .001 \\
\hline WV077 & $\mathbf{N}$ & 300 & $\mathbb{N}$ & 100 & $\mathbb{N}$ & 20 & $\mathrm{~N}$ & 300 & $<.001$ \\
\hline WV078 & $\mathbb{N}$ & 300 & N & 100 & $\mathbf{N}$ & 15 & N & 300 & .004 \\
\hline WV079 & $\mathrm{N}$ & 300 & N & 100 & N & 20 & N & 300 & .001 \\
\hline WW080 & $\mathbb{N}$ & 300 & $\mathrm{~N}$ & 70 & $\mathbf{N}$ & 10 & N & 200 & .003 \\
\hline WV081 & $\mathbf{N}$ & 300 & $\mathbb{N}$ & 150 & N & 10 & $\mathbb{N}$ & 150 & .001 \\
\hline WV082 & $\mathbb{N}$ & 300 & N & 100 & $\mathrm{~N}$ & 15 & N & 200 & $<.001$ \\
\hline WV083 & $\mathbb{N}$ & 300 & $\mathbb{N}$ & 70 & $\mathbb{N}$ & 15 & N & 150 & $<.001$ \\
\hline WV084 & $\mathbf{N}$ & 500 & N & 100 & $\mathbb{N}$ & 15 & $\mathrm{~N}$ & 200 & $<.001$ \\
\hline WV085 & $\mathrm{N}$ & 300 & $\mathbb{N}$ & 100 & $\mathbf{N}$ & 15 & $\mathbb{N}$ & 200 & .001 \\
\hline WV086 & $\mathbf{N}$ & 300 & $\mathbb{N}$ & 150 & $\mathbb{N}$ & 20 & N & 300 & $<.001$ \\
\hline WV087 & $\mathbf{N}$ & 300 & $\mathbb{N}$ & 150 & $\mathbf{N}$ & 15 & $<200$ & 70 & .01 \\
\hline WV088 & $\mathbb{N}$ & 300 & N & 200 & $\mathbb{N}$ & 20 & $<200$ & 200 & .001 \\
\hline WV089 & $\mathrm{N}$ & 300 & N & 150 & $N$ & 20 & $<200$ & 300 & .001 \\
\hline WV090 & $\mathbb{N}$ & 300 & N & 200 & N & 15 & $<200$ & 100 & .006 \\
\hline WV091 & $\mathbf{N}$ & 300 & $\mathbb{N}$ & 150 & N & 15 & $\mathrm{~N}$ & 150 & $<.001$ \\
\hline WV092 & $\mathrm{N}$ & 300 & $\mathbb{N}$ & 150 & $\mathbb{N}$ & 15 & $<200$ & 150 & .01 \\
\hline WV093 & $\mathbb{N}$ & 300 & N & 150 & $\mathrm{~N}$ & 15 & $<200$ & 100 & .001 \\
\hline WV094 & $\mathrm{N}$ & 500 & N & 70 & $\mathbb{N}$ & 15 & $\mathrm{~N}$ & 150 & $<.001$ \\
\hline WV095 & $\mathbf{N}$ & 300 & N & 150 & N & 20 & N & 200 & .001 \\
\hline WV096 & $N$ & 300 & N & 200 & N & 15 & N & 200 & .001 \\
\hline WV097 & $\mathbb{N}$ & 300 & $\mathbb{N}$ & 150 & $\mathbb{N}$ & 15 & N & 300 & .001 \\
\hline WV098 & $\mathrm{N}$ & 300 & $N$ & 150 & $\mathrm{~N}$ & 15 & N & 300 & .003 \\
\hline WV099 & $\mathbb{N}$ & 500 & $\mathbb{N}$ & 150 & $\mathrm{~N}$ & 15 & N & 200 & .005 \\
\hline WV100 & $\mathbf{N}$ & 300 & N & 150 & N & 20 & $<200$ & 300 & .001 \\
\hline WV101 & $\mathbb{N}$ & 500 & $N$ & 150 & $\mathbb{N}$ & 20 & $<200$ & 150 & .001 \\
\hline WV102 & N & 300 & $\mathbb{N}$ & 100 & $\mathbb{N}$ & 15 & $<200$ & 100 & .54 \\
\hline
\end{tabular}


Table 2. Gold and spectrographic analyses of 238 B-horizon soil samples.--Continued

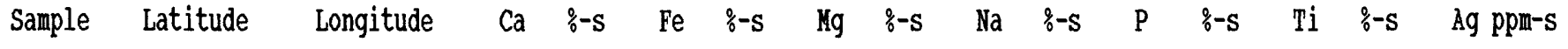

\begin{tabular}{|c|c|c|c|c|c|c|c|c|c|}
\hline WW103 & 475050 & 922326 & .5 & 3 & .7 & 2 & .2 & .5 & .7 \\
\hline WV104 & 475057 & 922355 & .3 & 3 & .7 & 1.5 & .2 & .5 & .7 \\
\hline WV105 & $4751 \quad 17$ & 922257 & .7 & 3 & 1 & 2 & .3 & .5 & $<.5$ \\
\hline WV106 & $\begin{array}{lll}47 & 5127\end{array}$ & $92 \quad 22 \quad 4$ & .7 & 2 & .7 & 2 & .2 & .3 & $<.5$ \\
\hline W107 & 475135 & 922119 & .7 & 3 & 1 & 2 & .2 & .7 & $<.5$ \\
\hline WW108 & 475158 & 922050 & .5 & 3 & .7 & 1.5 & .3 & .5 & .5 \\
\hline WV109 & 475110 & 922429 & .5 & 2 & .7 & 1.5 & .2 & .3 & $<.5$ \\
\hline WV110 & 475122 & $9225 \quad 3$ & .5 & 3 & .7 & 1.5 & $<.2$ & .5 & $<.5$ \\
\hline WV111 & 475142 & 922533 & .5 & 3 & .7 & 1.5 & $<.2$ & .3 & $<.5$ \\
\hline W112 & $4752 \quad 1$ & 922512 & .3 & 3 & .7 & 1.5 & .5 & .5 & .5 \\
\hline WW113 & 47521 & 922433 & .5 & 3 & .7 & 1.5 & .2 & .5 & $<.5$ \\
\hline WW114 & 47524 & $9224 \quad 1$ & .5 & 3 & 1 & 1.5 & $<.2$ & .5 & $<.5$ \\
\hline WW115 & 475218 & 922320 & .5 & 3 & .7 & 2 & .2 & .5 & .7 \\
\hline WV116 & 475219 & 922244 & .5 & 3 & .7 & 1.5 & $<.2$ & .5 & $N$ \\
\hline WW117 & 475220 & 922115 & .3 & 3 & .7 & 1.5 & .2 & .7 & .7 \\
\hline WV118 & 475226 & 922218 & 1 & 5 & 1.5 & 3 & $<.2$ & .7 & $\mathrm{~N}$ \\
\hline WV119 & 475245 & 922238 & .5 & 3 & .7 & 1.5 & $<.2$ & .5 & $<.5$ \\
\hline WV120 & 475259 & 92239 & .5 & 3 & .7 & 1.5 & $<.2$ & .5 & $\mathbb{N}$ \\
\hline WV121 & 475321 & 922253 & .5 & 3 & .7 & 1.5 & .2 & .3 & $<.5$ \\
\hline WW122 & $\begin{array}{lll}47 & 5317\end{array}$ & 922336 & .7 & 2 & .7 & 1.5 & .2 & .3 & $N$ \\
\hline WW123 & 47530 & 922348 & .3 & 3 & .7 & 1.5 & .2 & .3 & $N$ \\
\hline WV124 & 47530 & 922414 & .5 & 2 & .7 & 1.5 & $<.2$ & .5 & $N$ \\
\hline WV125 & 475235 & 922453 & .5 & 2 & .7 & 1.5 & $<.2$ & .5 & $\mathrm{~N}$ \\
\hline WV126 & $\begin{array}{lll}47 & 53 & 8\end{array}$ & 922454 & .5 & 3 & .7 & 2 & $<.2$ & .5 & $<.5$ \\
\hline WV127 & 475236 & 922532 & .2 & 5 & 1.5 & 2 & .3 & .5 & $N$ \\
\hline WV128 & 475210 & 922641 & .3 & 3 & .7 & 1.5 & $<.2$ & .5 & $N$ \\
\hline WV129 & 475241 & 922652 & .3 & 2 & .7 & 1 & .2 & .3 & 1 \\
\hline WV130 & 475425 & 922858 & .5 & 3 & .7 & 1.5 & $<.2$ & .3 & $\mathbb{N}$ \\
\hline WV131 & $4754 \quad 42$ & $9229 \quad 2$ & .5 & 3 & .7 & 1.5 & $<.2$ & .3 & $N$ \\
\hline WV132 & 475459 & 922858 & .5 & 3 & .7 & 1.5 & .2 & .5 & $\mathrm{~N}$ \\
\hline WV133 & 475353 & 922859 & .3 & 3 & .7 & 2 & $<.2$ & .3 & $<.5$ \\
\hline WW134 & 475336 & 922839 & .3 & 3 & .5 & 1 & .2 & .5 & $<.5$ \\
\hline W135 & 475434 & 922829 & .5 & 3 & .7 & 1.5 & $<.2$ & .3 & $\mathbb{N}$ \\
\hline WV136 & $4754 \quad 7$ & 922927 & .5 & 3 & .7 & 1.5 & $<.2$ & .5 & $N$ \\
\hline WV137 & 475353 & $92 \quad 2959$ & .5 & 3 & .7 & 1.5 & $<.2$ & .5 & $\mathrm{~N}$ \\
\hline WW138 & 475341 & 922928 & .5 & 3 & .7 & 1.5 & $<.2$ & .3 & $\mathbb{N}$ \\
\hline WV139 & 475328 & $92 \quad 2919$ & .3 & 2 & .7 & 1.5 & $<.2$ & .5 & $N$ \\
\hline WW140 & 475353 & 923036 & .5 & 2 & 1 & 1.5 & $<.2$ & .3 & $N$ \\
\hline WV141 & 475329 & $9230 \quad 6$ & .3 & 3 & 1 & 1.5 & $<.2$ & .5 & N \\
\hline WW142 & $4753 \quad 14$ & $92 \quad 2912$ & .5 & 3 & .7 & 1.5 & .2 & .7 & $<.5$ \\
\hline WV143 & $4753 \quad 42$ & 923110 & .5 & 1.5 & .5 & 1.5 & $<.2$ & .2 & $\mathbb{N}$ \\
\hline WV144 & 475328 & 923143 & .7 & 2 & .7 & 2 & $<.2$ & .3 & $N$ \\
\hline W145 & 475357 & 923226 & .5 & 1.5 & .3 & 1.5 & $<.2$ & .5 & $<.5$ \\
\hline W146 & 475333 & 923222 & .7 & 2 & .7 & 2 & $<.2$ & .5 & $\mathrm{~N}$ \\
\hline WV147 & 475339 & 923260 & .7 & 2 & .7 & 2 & $<.2$ & .5 & $N$ \\
\hline WV148 & $4753 \quad 43$ & 923335 & .5 & 3 & .7 & 2 & .2 & .5 & $<.5$ \\
\hline WV149 & 475425 & 923423 & .7 & 2 & .7 & 1.5 & $<.2$ & .3 & $<.5$ \\
\hline W150 & $4754 \quad 15$ & 92356 & .7 & 3 & .7 & 2 & $<.2$ & .5 & $N$ \\
\hline WV151 & 475437 & 923458 & .7 & 3 & .7 & 1.5 & $<.2$ & .5 & $\mathbb{N}$ \\
\hline WV152 & 47543 & 923534 & .7 & 2 & .5 & 2 & $<.2$ & .15 & $N$ \\
\hline
\end{tabular}


Table 2. Gold and spectrographic analyses of 238 B-horizon soil samples,--Continued

Sample As ppm-s B ppm-s Ba ppm-s Be ppm-s Bi ppm-s cd ppm-s co ppm-s cr ppm-s cu ppm-s

\begin{tabular}{|c|c|c|c|c|c|c|c|c|c|}
\hline WV103 & N & 30 & 700 & 1 & $N$ & $N$ & 20 & 150 & 20 \\
\hline WV104 & N & 30 & 700 & 1 & N & N & 20 & 150 & 20 \\
\hline WV105 & $\mathbf{N}$ & 20 & 700 & 1.5 & $\mathbf{N}$ & $\mathrm{N}$ & 20 & 300 & 30 \\
\hline WV106 & $\mathbf{N}$ & 15 & 700 & 1.5 & $\mathbf{N}$ & N & 15 & 70 & 15 \\
\hline WV107 & $\mathbf{N}$ & 20 & 1,000 & 1 & N & $\mathbf{N}$ & 20 & 200 & 20 \\
\hline WV108 & N & 30 & 1,000 & 1 & $N$ & N & 20 & 300 & 30 \\
\hline WV109 & N & 15 & 700 & 1 & N & N & $<10$ & 70 & 15 \\
\hline WW110 & $\mathbb{N}$ & 20 & 1,000 & 1 & $\mathrm{~N}$ & N & 15 & 150 & 15 \\
\hline WW111 & $N$ & 50 & 700 & 1.5 & $\mathbf{N}$ & $\mathbf{N}$ & 15 & 100 & 20 \\
\hline WI112 & $\mathbf{N}$ & 30 & 1,000 & 1 & $\mathbf{N}$ & N & 15 & 100 & 30 \\
\hline WV113 & $\mathbf{N}$ & 30 & 700 & 1 & N & $\mathbf{N}$ & 30 & 150 & 30 \\
\hline WV114 & N & 30 & 1,000 & 1 & N & $\mathbf{N}$ & 30 & 150 & 20 \\
\hline WW115 & $\mathbb{N}$ & 30 & 1,000 & 1 & N & N & 30 & 150 & 30 \\
\hline WV116 & $\mathbf{N}$ & 30 & 700 & $<1$ & $\mathrm{~N}$ & $\mathbf{N}$ & 20 & 150 & 20 \\
\hline WW117 & $\mathbb{N}$ & 50 & 700 & 1 & N & N & 15 & 100 & 30 \\
\hline WV118 & $\mathrm{N}$ & $<10$ & 500 & 1 & $N$ & $\mathbf{N}$ & 50 & 150 & 50 \\
\hline WW119 & $\mathrm{N}$ & 50 & 1,000 & 1 & $\mathbf{N}$ & $\mathbf{N}$ & 20 & 150 & 15 \\
\hline WV120 & N & 50 & 1,000 & 1 & N & $N$ & 20 & 100 & 15 \\
\hline WV121 & $\mathbb{N}$ & 20 & 1,000 & 1 & $\mathrm{~N}$ & $\mathbf{N}$ & 20 & 150 & 20 \\
\hline WW122 & $\mathrm{N}$ & 15 & 1,500 & 1 & $\mathrm{~N}$ & N & 15 & 100 & 15 \\
\hline WV123 & $N$ & 20 & 700 & $<1$ & N & N & 20 & 150 & 20 \\
\hline WV124 & $\mathrm{N}$ & 30 & 700 & 1 & N & $\mathrm{N}$ & 20 & 100 & 20 \\
\hline WW125 & $\mathbb{N}$ & 20 & 700 & 1 & $\mathbf{N}$ & N & 15 & 100 & 15 \\
\hline WV126 & $\mathbb{N}$ & 30 & 1,000 & 1 & $\mathrm{~N}$ & $\mathrm{~N}$ & 20 & 150 & 20 \\
\hline WV127 & $\mathbf{N}$ & 15 & 500 & 1 & N & $\mathbf{N}$ & 50 & 700 & 20 \\
\hline WV128 & $\mathrm{N}$ & 20 & 700 & $<1$ & $\mathbf{N}$ & $\mathbf{N}$ & 20 & 150 & 15 \\
\hline WV129 & $\mathbf{N}$ & 30 & 1,000 & 1 & N & $\mathbf{N}$ & 20 & 100 & 20 \\
\hline WN130 & $N$ & 20 & 700 & 1 & $N$ & N & 20 & 100 & 15 \\
\hline W131 & $\mathbf{N}$ & 20 & 700 & 1 & $\mathbf{N}$ & N & 20 & 100 & 15 \\
\hline WV132 & $N$ & 20 & 700 & 1 & $\mathbf{N}$ & $\mathbf{N}$ & 30 & 100 & 15 \\
\hline WV133 & $\mathbf{N}$ & 20 & 700 & 1 & N & $\mathbf{N}$ & 20 & 150 & 20 \\
\hline WV134 & $\mathbf{N}$ & 30 & 500 & $<1$ & $\mathbf{N}$ & $\mathbf{N}$ & $<10$ & 100 & 20 \\
\hline WV135 & $\mathbb{N}$ & 30 & 700 & 1 & N & N & 15 & 100 & 20 \\
\hline WV136 & $\mathbb{N}$ & 20 & 1,000 & 1 & N & N & 20 & 100 & 20 \\
\hline WV137 & $\mathbb{N}$ & 20 & 1,000 & $<1$ & $\mathrm{~N}$ & $\mathbf{N}$ & 20 & 100 & 15 \\
\hline WV138 & $\mathbf{N}$ & 20 & 700 & $<1$ & $\mathrm{~N}$ & N & 20 & 100 & 20 \\
\hline WV139 & N & 20 & 700 & $<1$ & N & N & 20 & 100 & 15 \\
\hline WV140 & $N$ & 15 & 700 & 1 & N & $\mathbf{N}$ & 20 & 100 & 15 \\
\hline WV141 & $\mathbf{N}$ & 30 & 700 & $<1$ & $\mathbf{N}$ & $\mathbf{N}$ & 30 & 100 & 20 \\
\hline WD142 & $\mathrm{N}$ & 30 & 700 & $<1$ & N & $\mathbf{N}$ & 30 & 150 & 20 \\
\hline WV143 & $\mathbf{N}$ & 20 & 700 & 1 & $\mathbf{N}$ & $\mathbf{N}$ & 10 & 50 & 10 \\
\hline WV144 & $\mathbf{N}$ & 20 & 700 & 1 & $\mathrm{~N}$ & N & $<10$ & 70 & 10 \\
\hline WV145 & $\mathbf{N}$ & 20 & 1,000 & $<1$ & $\mathbf{N}$ & N & N & 70 & 10 \\
\hline WV146 & $\mathbf{N}$ & 20 & 1,000 & 1 & N & $\mathbf{N}$ & $<10$ & 70 & 15 \\
\hline WV147 & $\mathbf{N}$ & 15 & 1,000 & 1 & $\mathrm{~N}$ & $\mathbb{N}$ & 10 & 70 & 15 \\
\hline WV148 & $\mathbb{N}$ & 20 & 1,000 & 1 & N & $\mathrm{N}$ & 15 & 100 & 15 \\
\hline WV149 & $\mathbf{N}$ & 20 & 700 & 1 & N & N & $<10$ & 70 & 15 \\
\hline WV150 & N & 30 & 1,000 & $<1$ & $\mathbf{N}$ & $\mathbf{N}$ & 30 & 150 & 20 \\
\hline WV151 & $\mathbf{N}$ & 30 & 700 & $<1$ & $\mathrm{~N}$ & $\mathbf{N}$ & 20 & 150 & 15 \\
\hline WW152 & $\mathbf{N}$ & 15 & 700 & 1 & $\mathbf{N}$ & $\mathbf{N}$ & $<10$ & 70 & 15 \\
\hline
\end{tabular}


Table 2. Gold and spectrographic analyses of 238 B-horizon soil samples.--Continued

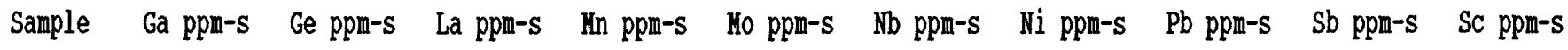

\begin{tabular}{|c|c|c|c|c|c|c|c|c|c|c|}
\hline WV103 & 50 & N & 50 & 700 & $\mathbb{N}$ & $<20$ & 50 & 50 & N & 7 \\
\hline WV104 & 30 & $\mathrm{~N}$ & $<50$ & 500 & N & $<20$ & 50 & 30 & $\mathbf{N}$ & 7 \\
\hline WV105 & 50 & $\mathbf{N}$ & $<50$ & 700 & N & $<20$ & 50 & 50 & N & 7 \\
\hline WV106 & 30 & N & $<50$ & 700 & $\mathrm{~N}$ & $<20$ & 20 & 30 & $\mathbf{N}$ & 7 \\
\hline W107 & 50 & $\mathbb{N}$ & 50 & 1,500 & N & 20 & 50 & 30 & $\mathbb{N}$ & 7 \\
\hline WV108 & 50 & N & $<50$ & 3,000 & $\mathbb{N}$ & $<20$ & 50 & 50 & $\mathbf{N}$ & 7 \\
\hline WV109 & 50 & $\mathbf{N}$ & $<50$ & 300 & N & $<20$ & 30 & 30 & $\mathbf{N}$ & 5 \\
\hline WV110 & 50 & N & $<50$ & 500 & N & $<20$ & 50 & 30 & $\mathbf{N}$ & 7 \\
\hline WV111 & 50 & $\mathbf{N}$ & 50 & 700 & N & $<20$ & 50 & 50 & N & 7 \\
\hline WV112 & 50 & N & $<50$ & 1,500 & N & 20 & 50 & 70 & $N$ & 7 \\
\hline W113 & 30 & $\mathbf{N}$ & 50 & 2,000 & N & $<20$ & 50 & 50 & $\mathbb{N}$ & 7 \\
\hline WV114 & 50 & N & $<50$ & 500 & $\mathbb{N}$ & $<20$ & 50 & 50 & N & 10 \\
\hline WV115 & 50 & N & 50 & 1,000 & $N$ & $<20$ & 50 & 50 & $\mathbb{N}$ & 10 \\
\hline WV116 & 30 & N & $<50$ & 300 & N & $<20$ & 50 & 30 & N & 10 \\
\hline WV117 & 50 & $\mathbb{N}$ & 50 & 700 & N & 20 & 30 & 50 & $N$ & 10 \\
\hline WV118 & 70 & N & $<50$ & 1,500 & $\mathbb{N}$ & $<20$ & 50 & 30 & $\mathbf{N}$ & 30 \\
\hline WV119 & 50 & N & 50 & 500 & N & $<20$ & 50 & 30 & N & 10 \\
\hline WV120 & 50 & $\mathbf{N}$ & 50 & 700 & N & $<20$ & 50 & 30 & $\mathbb{N}$ & 10 \\
\hline WV121 & 50 & N & $<50$ & 500 & N & $<20$ & 50 & 50 & $\mathbf{N}$ & 7 \\
\hline WV122 & 30 & $\mathrm{~N}$ & $<50$ & 700 & $\mathbb{N}$ & $<20$ & 50 & 30 & N & 7 \\
\hline WV123 & 30 & N & $<50$ & 300 & N & $<20$ & 50 & 30 & $\mathbf{N}$ & 7 \\
\hline WV124 & 50 & $\mathbf{N}$ & $<50$ & 700 & $\mathbb{N}$ & $<20$ & 30 & 30 & $\mathbf{N}$ & 7 \\
\hline WV125 & 30 & N & $<50$ & 300 & N & $<20$ & 30 & 30 & $\mathbb{N}$ & 7 \\
\hline WV126 & 50 & N & $<50$ & 1,500 & N & 20 & 50 & 50 & $\mathbf{N}$ & 7 \\
\hline WV127 & 50 & N & $<50$ & 700 & $\mathrm{~N}$ & $<20$ & 100 & 50 & $\mathbf{N}$ & 10 \\
\hline WV128 & 50 & N & $<50$ & 300 & $\mathrm{~N}$ & $<20$ & 50 & 50 & $\mathbf{N}$ & 7 \\
\hline WV129 & 30 & $\mathbb{N}$ & $<50$ & 1,500 & N & $<20$ & 30 & 50 & $\mathbb{N}$ & 7 \\
\hline WV130 & 50 & $\mathbf{N}$ & $<50$ & 300 & $\mathbf{N}$ & $<20$ & 30 & 30 & N & 7 \\
\hline WV131 & 50 & $\mathbb{N}$ & $<50$ & 700 & N & $<20$ & 30 & 50 & $\mathbb{N}$ & 7 \\
\hline WV132 & 50 & N & $<50$ & 700 & $<5$ & $<20$ & 30 & 30 & N & 7 \\
\hline WV133 & 50 & N & $<50$ & 300 & N & $<20$ & 30 & 30 & N & 7 \\
\hline WV134 & 50 & $\mathbf{N}$ & $<50$ & 500 & N & 20 & 20 & 50 & N & 5 \\
\hline WV135 & 50 & $\mathbb{N}$ & $<50$ & 300 & $\mathrm{~N}$ & $<20$ & 50 & 50 & $\mathrm{~N}$ & 7 \\
\hline WV136 & 50 & N & $<50$ & 500 & $\mathrm{~N}$ & $<20$ & 50 & 30 & $\mathbb{N}$ & 7 \\
\hline WV137 & 50 & N & $<50$ & 500 & N & $<20$ & 50 & 50 & $N$ & 7 \\
\hline WV138 & 50 & N & $<50$ & 500 & N & $<20$ & 50 & 50 & $\mathrm{~N}$ & 7 \\
\hline WV139 & 50 & N & $<50$ & 700 & $N$ & $<20$ & 30 & 30 & $N$ & 7 \\
\hline WV140 & 50 & N & $<50$ & 300 & $\mathrm{~N}$ & $<20$ & 50 & 50 & $\mathbb{N}$ & 7 \\
\hline WV141 & 50 & $\mathrm{~N}$ & $<50$ & 500 & $\mathrm{~N}$ & $<20$ & 50 & 50 & $\mathbb{N}$ & 7 \\
\hline WV142 & 50 & N & $<50$ & 700 & $\mathrm{~N}$ & 20 & 50 & 50 & $\mathbb{N}$ & 10 \\
\hline WV143 & 30 & N & $<50$ & 200 & $\mathrm{~N}$ & $<20$ & 20 & 30 & N & 5 \\
\hline WV144 & 30 & N & $<50$ & 500 & $N$ & $<20$ & 20 & 30 & $\mathbb{N}$ & 5 \\
\hline WV145 & 20 & N & $<50$ & 150 & $\mathrm{~N}$ & $<20$ & 5 & 30 & $\mathrm{~N}$ & $<5$ \\
\hline WV146 & 30 & $\mathbf{N}$ & $<50$ & 300 & $N$ & $<20$ & 30 & 30 & $\mathbf{N}$ & 7 \\
\hline WV147 & 30 & $\mathbb{N}$ & 50 & 700 & N & $<20$ & 20 & 30 & $\mathrm{~N}$ & 7 \\
\hline WV148 & 50 & N & $<50$ & 700 & $\mathrm{~N}$ & $<20$ & 20 & 30 & N & 7 \\
\hline WV149 & 30 & $\mathbb{N}$ & $<50$ & 300 & $\mathbb{N}$ & $<20$ & 20 & 30 & N & 7 \\
\hline WV150 & 30 & N & 50 & 1,500 & $\mathrm{~N}$ & $<20$ & 30 & 30 & $\mathbf{N}$ & 7 \\
\hline WV151 & 30 & N & $<50$ & 1,500 & $N$ & $<20$ & 50 & 50 & $N$ & 10 \\
\hline WV152 & 30 & N & N & 300 & $N$ & N & 20 & 20 & N & 5 \\
\hline
\end{tabular}


Table 2. Gold and spectrographic analyses of 238 B-horizon soil samples.--Continued

Sample Sn ppm-s Sr ppm-s Th ppm-s $\quad$ V ppm-s W ppm-s Y ppm-s in ppm-s Ir ppm-s Au ppm-a

\begin{tabular}{|c|c|c|c|c|c|c|c|c|c|}
\hline WW103 & $N$ & 300 & $N$ & 100 & N & 20 & $<200$ & 300 & .001 \\
\hline WV104 & $N$ & 300 & $\mathbb{N}$ & 100 & $\mathrm{~N}$ & 15 & $<200$ & 200 & .001 \\
\hline WV105 & $N$ & 300 & $N$ & 100 & N & 15 & 200 & 70 & .002 \\
\hline WV106 & $\mathrm{N}$ & 500 & $\mathrm{~N}$ & 100 & N & 15 & $<200$ & 200 & .001 \\
\hline WV107 & $\mathbb{N}$ & 500 & $\mathrm{~N}$ & 150 & N & 20 & 200 & 300 & .003 \\
\hline WV108 & $\mathbb{N}$ & 300 & $N$ & 100 & N & 15 & $<200$ & 300 & .001 \\
\hline WV109 & $N$ & 300 & $\mathrm{~N}$ & 100 & N & 10 & $\mathrm{~N}$ & 70 & .002 \\
\hline WV110 & $\mathrm{N}$ & 300 & $\mathbf{N}$ & 100 & N & 15 & $\mathrm{~N}$ & 200 & .001 \\
\hline WV111 & $\mathrm{N}$ & 300 & $\mathrm{~N}$ & 150 & N & 15 & $\mathbb{N}$ & 70 & .001 \\
\hline WV112 & $N$ & 200 & $\mathbb{N}$ & 100 & N & 15 & $<200$ & 200 & $<.001$ \\
\hline WV113 & $N$ & 300 & $\mathrm{~N}$ & 100 & $N$ & 15 & $<200$ & 300 & .001 \\
\hline WV114 & $N$ & 300 & $\mathrm{~N}$ & 100 & $\mathbb{N}$ & 15 & $<200$ & 300 & .003 \\
\hline WV115 & $\mathrm{N}$ & 300 & $\mathrm{~N}$ & 150 & $N$ & 20 & $<200$ & 300 & .001 \\
\hline WV116 & $N$ & 300 & $\mathrm{~N}$ & 150 & $N$ & 15 & N & 200 & .001 \\
\hline WV117 & $\mathrm{N}$ & 300 & $\mathrm{~N}$ & 150 & $\mathrm{~N}$ & 20 & $<200$ & 300 & $<.001$ \\
\hline WV118 & $\mathbb{N}$ & 300 & $\mathrm{~N}$ & 300 & $\mathbb{N}$ & 30 & $<200$ & 100 & .004 \\
\hline WV119 & $\mathbb{N}$ & 300 & $\mathbf{N}$ & 150 & $\mathrm{~N}$ & 15 & $<200$ & 150 & .001 \\
\hline WV120 & $\mathbb{N}$ & 300 & $N$ & 150 & $\mathbf{N}$ & 20 & N & 300 & .001 \\
\hline W121 & $\mathbb{N}$ & 300 & $\mathbf{N}$ & 100 & $\mathrm{~N}$ & 15 & $<200$ & 150 & .002 \\
\hline WV122 & $\mathbb{N}$ & 500 & $\mathbb{N}$ & 70 & $\mathrm{~N}$ & 10 & N & 200 & $<.001$ \\
\hline WV123 & $\mathrm{N}$ & 300 & $\mathbf{N}$ & 100 & $\mathbb{N}$ & 15 & $<200$ & 150 & .002 \\
\hline WV124 & $\mathrm{N}$ & 200 & $\mathbf{N}$ & 100 & N & 15 & N & 200 & .001 \\
\hline WV125 & $N$ & 300 & $\mathbb{N}$ & 100 & $\mathrm{~N}$ & 15 & N & 200 & .001 \\
\hline WV126 & $\mathbb{N}$ & 300 & $\mathrm{~N}$ & 100 & $\mathrm{~N}$ & 15 & 200 & 150 & .001 \\
\hline WV127 & $\mathrm{N}$ & 150 & $\mathrm{~N}$ & 150 & $\mathrm{~N}$ & 20 & $<200$ & 70 & .001 \\
\hline WV128 & $\mathrm{N}$ & 300 & $\mathrm{~N}$ & 100 & $\mathbb{N}$ & 15 & N & 200 & $<.001$ \\
\hline WV129 & $\mathrm{N}$ & 200 & $\mathbb{N}$ & 100 & $\mathbb{N}$ & 15 & 200 & 150 & .003 \\
\hline W130 & $N$ & 300 & $\mathbb{N}$ & 70 & N & 15 & N & 100 & $<.001$ \\
\hline WV131 & $N$ & 300 & $\mathbf{N}$ & 100 & N & 10 & N & 100 & .001 \\
\hline WV132 & $N$ & 300 & $\mathbb{N}$ & 100 & N & 10 & $\mathrm{~N}$ & 200 & $<.001$ \\
\hline WV133 & $\mathrm{N}$ & 200 & $\mathrm{~N}$ & 100 & $\mathrm{~N}$ & $<10$ & N & 150 & .006 \\
\hline WV134 & $N$ & 150 & $N$ & 150 & $N$ & 15 & $<200$ & 150 & .003 \\
\hline WV135 & $N$ & 300 & $\mathrm{~N}$ & 150 & $\mathrm{~N}$ & 15 & $\mathrm{~N}$ & 150 & $<.001$ \\
\hline WV136 & $N$ & 300 & $\mathrm{~N}$ & 150 & $\mathbb{N}$ & 15 & N & 300 & .001 \\
\hline WV137 & $\mathrm{N}$ & 300 & $N$ & 150 & $\mathbb{N}$ & 15 & $\mathbb{N}$ & 200 & $<.001$ \\
\hline WV138 & $\mathrm{N}$ & 300 & $\mathrm{~N}$ & 100 & $\mathrm{~N}$ & 10 & N & 150 & .001 \\
\hline WV139 & $\mathrm{N}$ & 200 & $\mathrm{~N}$ & 100 & $\mathrm{~N}$ & 15 & N & 200 & $<.001$ \\
\hline WV140 & $N$ & 300 & $N$ & 70 & $\mathbf{N}$ & $<10$ & N & 100 & $<.001$ \\
\hline WV141 & $N$ & 300 & $\mathrm{~N}$ & 100 & $\mathrm{~N}$ & 15 & N & 200 & .002 \\
\hline WV142 & $\mathbb{N}$ & 300 & $\mathrm{~N}$ & 100 & $\mathrm{~N}$ & 20 & $<200$ & 300 & .001 \\
\hline WV143 & $\mathrm{N}$ & 300 & $\mathrm{~N}$ & 70 & $\mathrm{~N}$ & $<10$ & N & 70 & $<.001$ \\
\hline WV144 & $\mathrm{N}$ & 300 & $\mathrm{~N}$ & 70 & $\mathbb{N}$ & 15 & N & 150 & $<.001$ \\
\hline WV145 & $\mathrm{N}$ & 300 & $N$ & 50 & $\mathbb{N}$ & 15 & N & 200 & .001 \\
\hline WV146 & $\mathrm{N}$ & 300 & $\mathbf{N}$ & 70 & $\mathrm{~N}$ & 15 & $\mathbb{N}$ & 200 & .002 \\
\hline WV147 & $\mathrm{N}$ & 300 & $\mathbf{N}$ & 70 & $\mathrm{~N}$ & 15 & N & 150 & .001 \\
\hline WV148 & $\mathrm{N}$ & 300 & $\mathbf{N}$ & 70 & $\mathbf{N}$ & 15 & N & 150 & .001 \\
\hline WV149 & $N$ & 300 & $\mathrm{~N}$ & 70 & $N$ & 15 & $N$ & 100 & .002 \\
\hline WV150 & $\mathbb{N}$ & 300 & $\mathbf{N}$ & 150 & $\mathrm{~N}$ & 30 & N & 200 & .001 \\
\hline WV151 & $N$ & 300 & $\mathrm{~N}$ & 100 & $\mathrm{~N}$ & 15 & $<200$ & 300 & $<.001$ \\
\hline WV152 & $N$ & 300 & $\mathrm{~N}$ & 70 & $N$ & 10 & $N$ & 70 & .001 \\
\hline
\end{tabular}


Table 2. Gold and spectrographic analyses of 238 B-horizon soil samples.--Continued

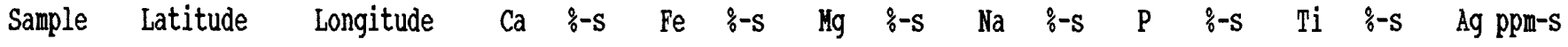

\begin{tabular}{|c|c|c|c|c|c|c|c|c|c|}
\hline WV153 & 475339 & 923523 & .5 & 2 & 1 & 1.5 & $<.2$ & .5 & N \\
\hline WV154 & 475322 & $9234 \quad 47$ & .7 & 3 & 1 & 2 & $<.2$ & .7 & $<.5$ \\
\hline WV155 & 475324 & 923525 & .7 & 3 & .7 & 2 & $<.2$ & .3 & N \\
\hline WV156 & 475254 & 923517 & .7 & 2 & .7 & 1.5 & $<.2$ & .3 & $N$ \\
\hline W157 & 475315 & 923555 & .7 & 2 & .7 & 1.5 & $<.2$ & .5 & $\mathrm{~N}$ \\
\hline WV158 & 475321 & 923645 & .7 & 3 & 1 & 2 & $<.2$ & .5 & $<.5$ \\
\hline WV159 & 475354 & 923639 & .7 & 2 & 1 & 2 & $<.2$ & .5 & $<.5$ \\
\hline WV160 & 475423 & 923648 & .5 & 2 & .5 & 1.5 & $<.2$ & .7 & N \\
\hline WV161 & 475245 & 923722 & .7 & 3 & 1 & 1.5 & $<.2$ & .7 & N \\
\hline W162 & 475134 & 923516 & .7 & 2 & .7 & 2 & .2 & .5 & N \\
\hline WV163 & $4752 \quad 4$ & 922820 & .7 & 3 & .7 & 2 & $<.2$ & .5 & N \\
\hline WV164 & 475157 & $92 \quad 2852$ & 1.5 & 3 & .7 & 3 & $<.2$ & .5 & N \\
\hline WV165 & 475128 & 922853 & .7 & 3 & 1 & 2 & $<.2$ & .5 & N \\
\hline WV166 & $4750 \quad 42$ & 923028 & .7 & 3 & .7 & 1.5 & .2 & .3 & $<.5$ \\
\hline WV167 & $4750 \quad 45$ & $9231 \quad 5$ & .7 & 3 & 1 & 1.5 & $<.2$ & .5 & .7 \\
\hline WV168 & $4753 \quad 27$ & $9237 \quad 24$ & 1.5 & 3 & 1 & 2 & $<.2$ & .5 & $<.5$ \\
\hline WV169 & $\begin{array}{lll}47 & 53 & 47\end{array}$ & 923755 & .7 & 3 & 1 & 5 & $<.2$ & .7 & $<.5$ \\
\hline WV170 & $4754 \quad 5$ & $9238 \quad 15$ & .5 & 1.5 & .5 & 1.5 & $<.2$ & .3 & $\mathrm{~N}$ \\
\hline W171 & 475426 & $9238 \quad 5$ & .7 & 3 & .7 & 1.5 & $<.2$ & 1 & $<.5$ \\
\hline W172 & 475451 & $9238 \quad 6$ & .7 & 1.5 & .3 & 1.5 & $<.2$ & .5 & $N$ \\
\hline WV173 & $4754 \quad 44$ & 923738 & 1.5 & 3 & 1 & 2 & $<.2$ & .7 & .5 \\
\hline WV174 & $\begin{array}{lll}47 & 55 & 1\end{array}$ & 923757 & .7 & 2 & .7 & 1.5 & .3 & .2 & $\mathrm{~N}$ \\
\hline W175 & 475427 & 923844 & .7 & 3 & 1 & 1.5 & $<.2$ & .7 & $<.5$ \\
\hline W176 & $4754 \quad 43$ & 923922 & .7 & 3 & 1 & 2 & $<.2$ & .7 & $N$ \\
\hline W177 & 475514 & 923926 & .7 & 3 & 1 & 1.5 & N & .7 & $\mathrm{~N}$ \\
\hline W178 & 475519 & 923855 & .7 & 2 & .7 & 1.5 & $<.2$ & .7 & $N$ \\
\hline WV179 & 475532 & 923848 & 1.5 & 3 & 1 & 2 & $<.2$ & .7 & $\mathrm{~N}$ \\
\hline WV180 & $4754 \quad 17$ & 923934 & .7 & 2 & .7 & 1.5 & $<.2$ & .5 & $\mathrm{~N}$ \\
\hline W181 & 475353 & 923934 & .7 & 5 & 1 & 1.5 & $N$ & .7 & $<.5$ \\
\hline WV182 & 475325 & 923759 & .7 & 5 & 1 & 1.5 & $<.2$ & .7 & $N$ \\
\hline W183 & 475239 & $92 \quad 4036$ & .7 & 5 & 1 & 1.5 & $<.2$ & 1 & $<.5$ \\
\hline WV184 & 475157 & $9241 \quad 12$ & .7 & 2 & .7 & 1.5 & $<.2$ & .5 & $\mathrm{~N}$ \\
\hline WV185 & 475141 & 924025 & .7 & 5 & 1 & 1.5 & N & .7 & N \\
\hline WV186 & $4752 \quad 5$ & 924026 & .7 & 5 & 1.5 & 1.5 & $<.2$ & .7 & N \\
\hline WV187 & 475141 & 923931 & .7 & 3 & 1 & 1.5 & $<.2$ & .7 & N \\
\hline WV188 & 47528 & $9239 \quad 4$ & .7 & 5 & 1 & 1 & $N$ & .7 & $\mathrm{~N}$ \\
\hline WV189 & 475144 & 923838 & .7 & 3 & 1 & 1.5 & $<.2$ & .7 & $N$ \\
\hline WV200 & 474659 & 923516 & 1 & 3 & 1 & 2 & $<.2$ & .7 & .5 \\
\hline WW201 & $\begin{array}{lll}47 & 47 & 3\end{array}$ & $9234 \quad 41$ & 1 & 2 & .7 & 2 & $<.2$ & .5 & .7 \\
\hline WW202 & 475421 & $92 \quad 2757$ & .7 & 2 & .7 & 1.5 & $<.2$ & .3 & N \\
\hline WV203 & 475358 & $92 \quad 2821$ & .7 & 2 & .5 & 2 & N & 1 & $\mathbb{N}$ \\
\hline WW204 & 475335 & $92 \quad 2811$ & .7 & 5 & .7 & 1.5 & .5 & .7 & N \\
\hline WV205 & $4753 \quad 48$ & $92 \quad 2738$ & .7 & 3 & 1 & 1.5 & $<.2$ & .7 & $N$ \\
\hline WV206 & $4754 \quad 1$ & $92 \quad 27 \quad 17$ & .3 & 1 & .3 & 1.5 & $<.2$ & .3 & N \\
\hline WW207 & 475331 & $92 \quad 27 \quad 1$ & .3 & 3 & .7 & 1 & .5 & .5 & N \\
\hline WW208 & $4753 \quad 40$ & $92 \quad 2646$ & .7 & 5 & 1 & 1.5 & .5 & .7 & $N$ \\
\hline WV209 & $47 \quad 53 \quad 13$ & $92 \quad 2722$ & .5 & 3 & .7 & 1.5 & 1 & .5 & .5 \\
\hline WW210 & 475257 & 922820 & 1 & 5 & 1 & 1.5 & .3 & 1 & $<.5$ \\
\hline WV211 & $4753 \quad 13$ & $92 \quad 2823$ & .7 & 3 & .7 & 1.5 & .5 & .5 & .7 \\
\hline WV212 & $4753 \quad 2$ & $92 \quad 2935$ & 1 & 1.5 & .7 & 1.5 & $<.2$ & .3 & N \\
\hline
\end{tabular}


Table 2. Gold and spectrographic analyses of 238 B-horizon soil samples.--Continued

Sample As ppm-s B ppm-s Ba ppm-s Be ppm-s Bi ppm-s Cd ppm-s Co ppm-s Cr ppm-s Cu ppm-s

\begin{tabular}{|c|c|c|c|c|c|c|c|c|c|}
\hline WV153 & N & 20 & 1,000 & 1 & $\mathrm{~N}$ & $\mathbf{N}$ & 15 & 150 & 10 \\
\hline WV154 & $\mathbb{N}$ & 30 & 1,000 & $<1$ & $N$ & $N$ & 15 & 150 & 20 \\
\hline WV155 & N & 30 & 1,000 & 1 & $\mathbb{N}$ & $\mathbb{N}$ & 15 & 100 & 15 \\
\hline WV:156 & N & 20 & 700 & 1 & $\mathbf{N}$ & N & 10 & 100 & 15 \\
\hline WV157 & $\mathbb{N}$ & 20 & 1,000 & 1 & $N$ & $N$ & 10 & 100 & 15 \\
\hline WV158 & $N$ & 30 & 1,000 & 1 & $N$ & $\mathbf{N}$ & 15 & 150 & 15 \\
\hline WV159 & $\mathrm{N}$ & 30 & 1,000 & 1 & $\mathrm{~N}$ & $\mathbb{N}$ & 15 & 100 & 15 \\
\hline WV160 & $N$ & 30 & 700 & $<1$ & N & $\mathrm{N}$ & 10 & 70 & 10 \\
\hline WV161 & $N$ & 30 & 1,000 & 1 & $N$ & $N$ & 20 & 150 & 15 \\
\hline WV162 & $N$ & 20 & 700 & 1 & $N$ & $\mathbb{N}$ & 15 & 100 & 15 \\
\hline WV163 & $N$ & 30 & 1,000 & 1 & $N$ & $\mathrm{~N}$ & 15 & 100 & 20 \\
\hline WV164 & $N$ & 30 & 1,000 & 1 & $N$ & $\mathrm{~N}$ & 15 & 100 & 20 \\
\hline WV165 & $N$ & 50 & 700 & 1 & $\mathrm{~N}$ & $\mathbf{N}$ & 20 & 150 & 20 \\
\hline WV166 & $N$ & 30 & 700 & 1 & $N$ & $\mathbf{N}$ & 15 & 70 & 15 \\
\hline WV167 & $\mathbb{N}$ & 50 & 700 & 1.5 & $N$ & $\mathbf{N}$ & 20 & 150 & 30 \\
\hline WV168 & $\mathrm{N}$ & 20 & 700 & 1 & $N$ & $\mathbf{N}$ & 20 & 200 & 20 \\
\hline WV169 & $\mathbb{N}$ & 30 & 1,000 & 1.5 & $\mathbb{N}$ & $\mathbb{N}$ & 15 & 150 & 15 \\
\hline WV170 & N & 20 & 700 & 1 & $\mathrm{~N}$ & $\mathbf{N}$ & $<10$ & 50 & 7 \\
\hline W171 & $\mathbb{N}$ & 50 & 1,000 & $<1$ & $N$ & $\mathbb{N}$ & 10 & 100 & 20 \\
\hline WV172 & $N$ & 30 & 700 & 1 & $N$ & $\mathbf{N}$ & $<10$ & 30 & 7 \\
\hline WV173 & N & 30 & 1,000 & 1 & $\mathbf{N}$ & $\mathbf{N}$ & 15 & 100 & 15 \\
\hline W174 & $N$ & 15 & 700 & 1.5 & $\mathbf{N}$ & $N$ & $<10$ & 100 & 10 \\
\hline WV175 & $N$ & 50 & 700 & 1 & $\mathbb{N}$ & $N$ & 15 & 150 & 20 \\
\hline WV176 & N & 50 & 1,000 & 1 & $N$ & $N$ & 20 & 150 & 15 \\
\hline W177 & $N$ & 30 & 1,000 & 1 & $\mathbb{N}$ & $\mathrm{N}$ & 20 & 100 & 20 \\
\hline WV178 & $N$ & 30 & 700 & 1 & $\mathbf{N}$ & $\mathbf{N}$ & 10 & 70 & 15 \\
\hline WV179 & $N$ & 30 & 1,000 & 1 & $\mathrm{~N}$ & $\mathrm{~N}$ & 20 & 150 & 15 \\
\hline WV180 & $\mathrm{N}$ & 20 & 700 & 1 & $\mathbb{N}$ & $\mathbf{N}$ & 20 & 100 & 15 \\
\hline WV181 & $N$ & 50 & 700 & 1 & $\mathbb{N}$ & $\mathrm{N}$ & 30 & 200 & 20 \\
\hline WV182 & $N$ & 30 & 700 & $<1$ & $\mathbb{N}$ & $N$ & 20 & 150 & 15 \\
\hline WV183 & $\mathrm{N}$ & 50 & 1,000 & 1 & $\mathrm{~N}$ & $\mathrm{~N}$ & 20 & 200 & 30 \\
\hline WV184 & $\mathbf{N}$ & 20 & 700 & 1 & $N$ & $\mathbb{N}$ & $<10$ & 70 & 15 \\
\hline WV185 & $\mathbf{N}$ & 50 & 1,000 & 1 & $N$ & $\mathrm{~N}$ & 20 & 200 & 30 \\
\hline WV186 & $\mathbf{N}$ & 70 & 1,000 & 1.5 & $\mathrm{~N}$ & $\mathbf{N}$ & 20 & 150 & 30 \\
\hline WV187 & $N$ & 50 & 1,000 & 1 & $N$ & $\mathrm{~N}$ & 15 & 150 & 20 \\
\hline WV188 & $\mathbb{N}$ & 70 & 1,000 & 1.5 & $N$ & $N$ & 15 & 150 & 30 \\
\hline WV189 & $N$ & 50 & 1,000 & 1.5 & $N$ & $\mathrm{~N}$ & 15 & 150 & 20 \\
\hline WV200 & $N$ & 50 & 1,000 & 1.5 & $N$ & $N$ & 20 & 150 & 15 \\
\hline W201 & $N$ & 50 & 1,000 & 1 & $N$ & $\mathbb{N}$ & $<10$ & 70 & 15 \\
\hline WV202 & $N$ & 20 & 1,000 & 1.5 & $\mathrm{~N}$ & $N$ & $<10$ & 50 & 15 \\
\hline WV203 & $N$ & 30 & 1,500 & $<1$ & $N$ & $N$ & N & 100 & 10 \\
\hline WV204 & $N$ & 30 & 1,000 & 1 & $N$ & $\mathbf{N}$ & 15 & 150 & 30 \\
\hline WV205 & $\mathrm{N}$ & 50 & 1,500 & 1 & $\mathrm{~N}$ & $\mathbb{N}$ & 20 & 100 & 15 \\
\hline WV206 & $N$ & 20 & 1,000 & $<1$ & $N$ & $N$ & $\mathrm{~N}$ & 30 & 10 \\
\hline WV207 & $\mathrm{N}$ & 20 & 1,000 & 1 & $N$ & $\mathrm{~N}$ & 10 & 70 & 20 \\
\hline WV208 & $\mathrm{N}$ & 30 & 1,000 & 1.5 & $\mathrm{~N}$ & $\mathrm{~N}$ & 20 & 150 & 20 \\
\hline WV209 & $N$ & 30 & 1,000 & 1 & N & $\mathrm{N}$ & 10 & 100 & 30 \\
\hline WV210 & $N$ & 30 & 1,500 & 1 & $N$ & $\mathrm{~N}$ & 10 & 150 & 30 \\
\hline WV211 & $N$ & 50 & 1,500 & 1.5 & $\mathrm{~N}$ & $\mathrm{~N}$ & 15 & 100 & 20 \\
\hline WV212 & $N$ & 20 & 500 & 1.5 & $N$ & $\mathrm{~N}$ & 10 & 100 & 20 \\
\hline
\end{tabular}


Table 2. Gold and spectrographic analyses of 238 B-horizon soil samples.--Continued

Sample Ga ppm-s Ge ppm-s La ppm-s Mn ppm-S Mo ppm-s Mb ppm-s Ni ppm-s Pb ppm-S Sb ppm-S Sc ppm-s

\begin{tabular}{|c|c|c|c|c|c|c|c|c|c|c|}
\hline WV153 & 30 & N & $<50$ & 300 & N & $<20$ & 30 & 30 & $\mathrm{~N}$ & 7 \\
\hline W154 & 30 & N & $<50$ & 500 & N & $<20$ & 50 & 50 & N & 10 \\
\hline WV155 & 50 & N & $<50$ & 300 & $\mathrm{~N}$ & $<20$ & 50 & 50 & N & 7 \\
\hline W156 & 30 & N & $<50$ & 500 & $N$ & $<20$ & 30 & 30 & $N$ & 7 \\
\hline WV157 & 30 & N & $<50$ & 500 & N & $<20$ & 30 & 30 & $\mathbf{N}$ & 7 \\
\hline WV158 & 50 & $\mathbb{N}$ & $<50$ & 500 & N & $<20$ & 50 & 30 & $\mathbb{N}$ & 10 \\
\hline WV159 & 50 & $\mathbb{N}$ & 50 & 700 & N & $<20$ & 50 & 30 & $\mathbf{N}$ & 7 \\
\hline WV160 & 30 & N & $<50$ & 300 & N & $<20$ & 15 & 20 & N & 7 \\
\hline WV161 & 50 & N & $<50$ & 700 & N & $<20$ & 50 & 30 & $\mathrm{~N}$ & 10 \\
\hline WV162 & 30 & N & $<50$ & 500 & $N$ & $<20$ & 30 & 30 & $N$ & 7 \\
\hline WV163 & 30 & N & 50 & 300 & N & $<20$ & 30 & 30 & $\mathbf{N}$ & 7 \\
\hline WV164 & 50 & N & 50 & 700 & $\mathbf{N}$ & $<20$ & 30 & 50 & $\mathbf{N}$ & 7 \\
\hline WV165 & 50 & N & $<50$ & 1,000 & N & $<20$ & 50 & 50 & $\mathbf{N}$ & 7 \\
\hline WV166 & 30 & $\mathrm{~N}$ & 70 & 500 & N & $<20$ & 30 & 30 & $\mathbf{N}$ & 7 \\
\hline WV167 & 50 & N & 50 & 1,000 & $N$ & $<20$ & 50 & 50 & N & 10 \\
\hline WV168 & 30 & $\mathrm{~N}$ & 50 & 700 & N & $<20$ & 50 & 30 & N & 7 \\
\hline WN169 & 50 & N & 50 & 500 & N & 20 & 50 & 50 & N & 10 \\
\hline WV170 & 30 & N & $<50$ & 300 & N & N & 20 & 30 & N & 5 \\
\hline WV171 & 30 & $\mathrm{~N}$ & $<50$ & 1,000 & $N$ & 20 & 30 & 50 & $\mathbf{N}$ & 7 \\
\hline WV172 & 30 & N & $<50$ & 300 & N & $<20$ & 15 & 20 & N & 5 \\
\hline WV173 & 50 & $N$ & $<50$ & 1,000 & $N$ & 20 & 30 & 30 & $N$ & 7 \\
\hline W174 & 30 & N & $<50$ & 500 & N & $<20$ & 20 & 30 & $\mathbf{N}$ & 5 \\
\hline WW175 & 30 & $N$ & 50 & 300 & $\mathbb{N}$ & $<20$ & 50 & 30 & $\mathbf{N}$ & 7 \\
\hline W176 & 50 & N & $<50$ & 500 & N & $<20$ & 50 & 50 & $\mathbf{N}$ & 10 \\
\hline WV177 & 50 & $\mathbf{N}$ & 50 & 700 & $\mathbb{N}$ & 20 & 50 & 50 & $\mathrm{~N}$ & 10 \\
\hline W178 & 30 & $\mathbf{N}$ & $<50$ & 500 & $\mathbb{N}$ & $<20$ & 30 & 30 & $\mathrm{~N}$ & 7 \\
\hline WV179 & 50 & N & $<50$ & 1,000 & $N$ & $<20$ & 30 & 50 & N & 10 \\
\hline WW180 & 30 & $\mathrm{~N}$ & $<50$ & 1,000 & $N$ & $<20$ & 30 & 50 & $\mathrm{~N}$ & 7 \\
\hline WV181 & 50 & $\mathrm{~N}$ & 50 & 700 & $\mathbb{N}$ & $<20$ & 50 & 50 & N & 10 \\
\hline WV182 & 50 & N & $<50$ & 500 & $\mathbb{N}$ & $<20$ & 50 & 50 & $\mathrm{~N}$ & 10 \\
\hline WV183 & 50 & N & 50 & 1,000 & $\mathbb{N}$ & $<20$ & 50 & 30 & $\mathrm{~N}$ & 10 \\
\hline WV184 & 30 & N & $<50$ & 500 & $\mathbf{N}$ & $<20$ & 20 & 30 & N & 7 \\
\hline WW185 & 50 & $\mathbf{N}$ & 50 & 1,000 & $\mathrm{~N}$ & $<20$ & 50 & 30 & $\mathbf{N}$ & 7 \\
\hline WV186 & 50 & N & 50 & 1,000 & $\mathbb{N}$ & $<20$ & 50 & 50 & N & 10 \\
\hline WV187 & 50 & N & $<50$ & 700 & $\mathbb{N}$ & $<20$ & 70 & 30 & N & 10 \\
\hline WV188 & 50 & $\mathrm{~N}$ & $<50$ & 700 & $\mathbb{N}$ & $<20$ & 50 & 30 & $\mathbf{N}$ & 10 \\
\hline W189 & 50 & $\mathrm{~N}$ & $<50$ & 700 & $\mathbf{N}$ & $<20$ & 50 & 50 & $N$ & 10 \\
\hline WV200 & 50 & N & $<50$ & 1,000 & N & $<20$ & 50 & 50 & N & 10 \\
\hline W201 & 50 & $\mathrm{~N}$ & $<50$ & 500 & $\mathbf{N}$ & $<20$ & 20 & 30 & $\mathbf{N}$ & 5 \\
\hline WW202 & 30 & N & $<50$ & 300 & N & $\mathbf{N}$ & 20 & 50 & N & 5 \\
\hline W203 & 50 & N & $<50$ & 300 & N & 20 & $<5$ & 50 & $\mathbf{N}$ & 7 \\
\hline WV204 & 50 & $\mathrm{~N}$ & 50 & 300 & $N$ & $<20$ & 30 & 50 & $\mathrm{~N}$ & 7 \\
\hline WV205 & 30 & $\mathrm{~N}$ & 50 & 1,000 & N & $<20$ & 30 & 30 & N & 7 \\
\hline WV206 & 30 & $\mathbf{N}$ & $<50$ & 200 & $\mathbf{N}$ & N & 5 & 50 & $\mathbf{N}$ & $<5$ \\
\hline WV207 & 30 & N & $<50$ & 200 & $\mathbf{N}$ & $\mathbf{N}$ & 30 & 30 & $\mathbb{N}$ & 5 \\
\hline WW208 & 50 & $\mathrm{~N}$ & $<50$ & 500 & N & $<20$ & 50 & 50 & N & 7 \\
\hline WV209 & 50 & $\mathrm{~N}$ & $<50$ & 300 & $\mathbf{N}$ & $<20$ & 30 & 50 & N & 7 \\
\hline WV210 & 50 & N & 50 & 700 & $N$ & 20 & 30 & 50 & N & 10 \\
\hline WV211 & 50 & N & 50 & 700 & $\mathbf{N}$ & $<20$ & 30 & 50 & N & 10 \\
\hline WV212 & 20 & N & $<50$ & 300 & N & $<20$ & 30 & 30 & N & 7 \\
\hline
\end{tabular}


Table 2. Gold and spectrographic analyses of 238 B-horizon soil samples.--Continued

Sample Sn ppm-s Sr ppm-s Th ppm-s $\quad V$ ppm-s W ppm-s $\quad$ Y ppm-s Zn ppm-s Ir ppm-s Au ppm-a

\begin{tabular}{|c|c|c|c|c|c|c|c|c|c|}
\hline WV153 & N & 300 & $N$ & 70 & N & 15 & $\mathrm{~N}$ & 300 & $<.001$ \\
\hline WV154 & $\mathrm{N}$ & 300 & $\mathrm{~N}$ & 70 & N & 15 & $\mathrm{~N}$ & 300 & $<.001$ \\
\hline WV155 & N & 300 & $\mathrm{~N}$ & 100 & $\mathbf{N}$ & 15 & $\mathrm{~N}$ & 150 & .009 \\
\hline WV156 & N & 300 & $\mathrm{~N}$ & 70 & $N$ & 10 & $\mathrm{~N}$ & 200 & .001 \\
\hline WV157 & $N$ & 300 & $\mathrm{~N}$ & 70 & $N$ & 15 & N & 200 & .001 \\
\hline WV158 & $N$ & 300 & $\mathrm{~N}$ & 100 & $\mathrm{~N}$ & 15 & N & 300 & $<.001$ \\
\hline WV159 & $\mathrm{N}$ & 300 & $\mathrm{~N}$ & 70 & $\mathrm{~N}$ & 20 & $\mathrm{~N}$ & 300 & $<.001$ \\
\hline WV160 & $\mathbf{N}$ & 300 & $N$ & 50 & $N$ & 15 & N & 300 & .001 \\
\hline WV161 & $\mathbf{N}$ & 300 & $\mathrm{~N}$ & 70 & $\mathrm{~N}$ & 20 & $\mathbf{N}$ & 200 & $<.001$ \\
\hline WV162 & $\mathrm{N}$ & 500 & $N$ & 70 & $N$ & 15 & N & 150 & $<.001$ \\
\hline WV163 & $\mathrm{N}$ & 500 & $\mathrm{~N}$ & 100 & $\mathrm{~N}$ & 15 & $N$ & 70 & .001 \\
\hline WV164 & $N$ & 700 & $\mathrm{~N}$ & 100 & $\mathrm{~N}$ & 15 & N & 200 & .002 \\
\hline WV165 & $\mathrm{N}$ & 500 & $\mathrm{~N}$ & 100 & $\mathrm{~N}$ & 20 & N & 150 & .002 \\
\hline W166 & $\mathrm{N}$ & 300 & $\mathrm{~N}$ & 70 & $\mathrm{~N}$ & 15 & $<200$ & 150 & $<.001$ \\
\hline WV167 & $\mathbb{N}$ & 300 & $\mathrm{~N}$ & 150 & $N$ & 15 & $<200$ & 200 & .001 \\
\hline WV168 & $N$ & 500 & $N$ & 100 & $\mathbb{N}$ & 20 & $\mathrm{~N}$ & 500 & $<.001$ \\
\hline WV169 & $N$ & 500 & $\mathrm{~N}$ & 100 & $\mathbf{N}$ & 20 & $\mathrm{~N}$ & 300 & .001 \\
\hline WV170 & $N$ & 300 & $\mathrm{~N}$ & 50 & $N$ & $<10$ & $N$ & 150 & $<.001$ \\
\hline WV171 & $N$ & 500 & $\mathrm{~N}$ & 100 & $\mathbf{N}$ & 20 & $<200$ & 500 & $<.001$ \\
\hline WV172 & $\mathrm{N}$ & 500 & $\mathrm{~N}$ & 70 & $N$ & $<10$ & N & 300 & .001 \\
\hline WV173 & $N$ & 500 & $N$ & 150 & $N$ & 15 & $<200$ & 500 & $<.001$ \\
\hline WV174 & $\mathrm{N}$ & 500 & $\mathrm{~N}$ & 70 & $\mathrm{~N}$ & 15 & N & 150 & .004 \\
\hline WV175 & $\mathrm{N}$ & 300 & $\mathbf{N}$ & 150 & $\mathbf{N}$ & 15 & $\mathrm{~N}$ & 300 & .001 \\
\hline WV176 & $\mathrm{N}$ & 500 & $N$ & 100 & $\mathrm{~N}$ & 20 & $\mathrm{~N}$ & 500 & $<.001$ \\
\hline WV177 & $N$ & 300 & $N$ & 150 & $\mathrm{~N}$ & 20 & $<200$ & 500 & $<.001$ \\
\hline WV178 & $\mathrm{N}$ & 300 & $\mathrm{~N}$ & 70 & $\mathrm{~N}$ & 15 & $<200$ & 300 & $<.001$ \\
\hline WV179 & $\mathbb{N}$ & 500 & $\mathrm{~N}$ & 100 & $\mathrm{~N}$ & 20 & $\mathrm{~N}$ & 300 & .001 \\
\hline WV180 & $\mathbb{N}$ & 500 & $\mathrm{~N}$ & 100 & $N$ & 15 & $\mathrm{~N}$ & 300 & $<.001$ \\
\hline WV181 & $\mathbb{N}$ & 300 & $\mathrm{~N}$ & 200 & $\mathrm{~N}$ & 15 & $\mathrm{~N}$ & 300 & .001 \\
\hline WV182 & $N$ & 300 & $\mathbf{N}$ & 150 & $\mathrm{~N}$ & 15 & $N$ & 300 & $<.001$ \\
\hline WV183 & $\mathbf{N}$ & 300 & $\mathrm{~N}$ & 200 & $\mathrm{~N}$ & 15 & $\mathbb{N}$ & 300 & .001 \\
\hline WV184 & $\mathbf{N}$ & 500 & $\mathrm{~N}$ & 70 & $\mathrm{~N}$ & $<10$ & $\mathrm{~N}$ & 100 & $<.001$ \\
\hline WV185 & $\mathbf{N}$ & 500 & $\mathrm{~N}$ & 150 & $\mathrm{~N}$ & 20 & $\mathrm{~N}$ & 300 & .001 \\
\hline WV186 & $\mathbb{N}$ & 300 & $\mathrm{~N}$ & 200 & $\mathrm{~N}$ & 20 & $\mathrm{~N}$ & 200 & .001 \\
\hline WV187 & $N$ & 300 & $N$ & 150 & $N$ & 15 & $\mathrm{~N}$ & 200 & .001 \\
\hline WV188 & $\mathrm{N}$ & 300 & $\mathbb{N}$ & 200 & $\mathrm{~N}$ & 15 & $\mathrm{~N}$ & 200 & .001 \\
\hline WV189 & $\mathbf{N}$ & 300 & $\mathrm{~N}$ & 150 & $\mathrm{~N}$ & 15 & $\mathrm{~N}$ & 200 & .001 \\
\hline WV200 & $\mathrm{N}$ & 500 & $\mathrm{~N}$ & 150 & $\mathbb{N}$ & 15 & $\mathrm{~N}$ & 200 & $<.001$ \\
\hline WV201 & $N$ & 500 & $N$ & 70 & $N$ & 10 & N & 200 & .001 \\
\hline WV202 & $N$ & 500 & $\mathrm{~N}$ & 70 & $\mathrm{~N}$ & $<10$ & $\mathrm{~N}$ & 150 & $<.001$ \\
\hline WV203 & $N$ & 500 & $\mathrm{~N}$ & 100 & $\mathrm{~N}$ & 20 & $\mathrm{~N}$ & 700 & $<.001$ \\
\hline WV204 & $\mathrm{N}$ & 300 & $\mathrm{~N}$ & 100 & $\mathrm{~N}$ & 20 & $\mathrm{~N}$ & 300 & .001 \\
\hline WV205 & $N$ & 500 & $\mathrm{~N}$ & 100 & $\mathrm{~N}$ & 20 & $\mathrm{~N}$ & 300 & .001 \\
\hline WV206 & $N$ & 300 & $N$ & 50 & $\mathrm{~N}$ & $<10$ & $\mathrm{~N}$ & 300 & $<.001$ \\
\hline WV207 & $\mathrm{N}$ & 200 & $\mathrm{~N}$ & 70 & $\mathbf{N}$ & 15 & $\mathrm{~N}$ & 200 & $<.001$ \\
\hline WV208 & $\mathbf{N}$ & 300 & $\mathrm{~N}$ & 150 & $\mathrm{~N}$ & 20 & $<200$ & 300 & .001 \\
\hline WV209 & N & 300 & $\mathrm{~N}$ & 150 & N & 15 & $\mathrm{~N}$ & 300 & $<.001$ \\
\hline WV210 & $\mathrm{N}$ & 500 & $\mathbf{N}$ & 150 & $\mathrm{~N}$ & 20 & $\mathrm{~N}$ & 500 & $<.001$ \\
\hline WV211 & $N$ & 300 & $N$ & 150 & $N$ & 20 & $N$ & 300 & $<.001$ \\
\hline WV212 & $\mathbf{N}$ & 300 & $\mathrm{~N}$ & 70 & $\mathrm{~N}$ & 10 & $\mathbb{N}$ & 100 & .001 \\
\hline
\end{tabular}


Table 2. Gold and spectrographic analyses of 238 B-horizon soil samples.--Continued

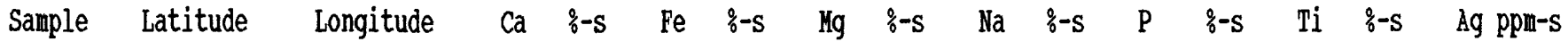

\begin{tabular}{|c|c|c|c|c|c|c|c|c|c|}
\hline WN213 & $4752 \quad 41$ & 922956 & 2 & 3 & 1 & 1.5 & .2 & .7 & N \\
\hline WV214 & 475221 & $92 \quad 2934$ & .7 & 3 & .7 & 1.5 & .2 & .5 & $<.5$ \\
\hline WV215 & $47 \quad 52 \quad 13$ & $92 \quad 2857$ & .7 & 2 & .7 & 1.5 & .2 & .3 & N \\
\hline WV216 & $4752 \quad 3$ & 922715 & .7 & 2 & .7 & 1.5 & $<.2$ & .3 & N \\
\hline WV218 & 475224 & 922755 & .7 & 3 & .7 & 1.5 & .2 & .5 & $<.5$ \\
\hline WV219 & $4753 \quad 16$ & $92 \quad 2636$ & .7 & 2 & .7 & 1.5 & $<.2$ & .5 & $<.5$ \\
\hline WV220 & $4753 \quad 34$ & 922515 & .7 & 3 & .7 & 1.5 & $<.2$ & .7 & $<.5$ \\
\hline WV221 & $4753 \quad 39$ & $92 \quad 2433$ & 1 & 3 & .7 & 2 & $<.2$ & 1 & $<.5$ \\
\hline WV222 & $\begin{array}{lll}47 & 49 & 8\end{array}$ & 922655 & 7 & .7 & .15 & .5 & 7 & .1 & N \\
\hline WV223 & 474916 & $92 \quad 2354$ & 1 & 3 & 1 & 1.5 & $<.2$ & .7 & $<.5$ \\
\hline WV224 & 474924 & $9224 \quad 7$ & 2 & 2 & 1 & 2 & $<.2$ & .3 & $<.5$ \\
\hline WV225 & $47 \quad 4942$ & 922413 & .5 & 3 & .7 & 1.5 & .2 & .5 & N \\
\hline WN226 & $47 \quad 4957$ & $92 \quad 24 \quad 24$ & 1.5 & 3 & .1 & 2 & $<.2$ & 1 & $\mathrm{~N}$ \\
\hline WN227 & 474956 & $92 \quad 2451$ & 2 & 3 & 1.5 & 2 & $<.2$ & .5 & N \\
\hline WN228 & 475435 & $9233 \quad 9$ & .7 & 2 & .7 & 2 & $<.2$ & .7 & $\mathrm{~N}$ \\
\hline WV229 & $4754 \quad 17$ & 923229 & .7 & 2 & .5 & 1.5 & .3 & .3 & $N$ \\
\hline WV230 & $4754 \quad 14$ & $92 \quad 34 \quad 37$ & .7 & 3 & .7 & 1.5 & 1 & .5 & $N$ \\
\hline WV231 & $4754 \quad 37$ & 923157 & 1.5 & 1.5 & .7 & 2 & $<.2$ & .3 & $\mathrm{~N}$ \\
\hline WV232 & $4754 \quad 24$ & 923121 & .7 & 3 & .7 & 1 & .7 & .7 & $<.5$ \\
\hline WV233 & $4755 \quad 2$ & 923119 & 1 & 3 & .7 & 1.5 & $<.2$ & .7 & $\mathrm{~N}$ \\
\hline WW234 & $47 \quad 55 \quad 41$ & $92 \quad 3046$ & .7 & 3 & .7 & 1.5 & 1 & .3 & $N$ \\
\hline WV235 & 475525 & $92 \quad 2949$ & 3 & 3 & 1 & 2 & .5 & 1 & $\mathrm{~N}$ \\
\hline WN236 & 475518 & $92 \quad 28 \quad 39$ & 3 & 2 & .7 & 2 & $<.2$ & .7 & .5 \\
\hline WV237 & 475514 & $92 \quad 28 \quad 1$ & .7 & 3 & .7 & 1.5 & $N$ & .5 & $\mathrm{~N}$ \\
\hline WV238 & 475438 & $92 \quad 2934$ & .7 & 3 & .7 & 1.5 & .2 & .7 & $\mathrm{~N}$ \\
\hline WV239 & $4754 \quad 17$ & $9230 \quad 4$ & 1.5 & 3 & 1 & 2 & .5 & .5 & N \\
\hline WV240 & 475359 & 923159 & .7 & 2 & .7 & 1.5 & .7 & .5 & N \\
\hline WV241 & $4754 \quad 28$ & 923330 & .3 & 3 & .5 & 1.5 & .3 & .3 & $\mathrm{~N}$ \\
\hline WV242 & $4754 \quad 41$ & 923359 & .5 & 1.5 & .5 & 1.5 & .3 & .2 & $<.5$ \\
\hline WN243 & 475125 & 921611 & .3 & 2 & .3 & 1 & 1.5 & .5 & 1.5 \\
\hline WV244 & $4751 \quad 18$ & 92160 & .7 & 3 & .7 & 1.5 & .2 & .7 & 1.5 \\
\hline WV245 & $4751 \quad 14$ & 922556 & .5 & 3 & .7 & 1.5 & .5 & .5 & 3 \\
\hline WN246 & $4751 \quad 17$ & 92164 & .7 & 3 & .7 & 1.5 & .2 & 1 & 3 \\
\hline WV247 & 475124 & $9216 \quad 6$ & .5 & 2 & .7 & 1.5 & .3 & .3 & 2 \\
\hline WV248 & $4751 \quad 10$ & $92 \quad 1611$ & 1.5 & 3 & .7 & 2 & $<.2$ & .7 & 3 \\
\hline WV249 & 475134 & 921136 & .5 & 2 & .7 & 1.5 & $<.2$ & .5 & $<.5$ \\
\hline WV261 & 475354 & 921534 & .7 & 3 & 1 & 1.5 & $<.2$ & .7 & $<.5$ \\
\hline WV268 & $4750 \quad 46$ & $92 \quad 13 \quad 12$ & .3 & 3 & .5 & 1 & .5 & .3 & $<.5$ \\
\hline
\end{tabular}


Table 2. Gold and spectrographic analyses of 238 B-horizon soil samples.--Continued

Sample As ppm-s B ppm-s Ba ppm-s Be ppm-s Bi ppm-s Cd ppm-s Co ppm-s Cr ppm-s Cu ppm-s

\begin{tabular}{|c|c|c|c|c|c|c|c|c|c|}
\hline WV213 & N & 30 & 1,000 & 1 & $\mathrm{~N}$ & $\mathrm{~N}$ & 15 & 100 & 15 \\
\hline WV214 & $\mathbb{N}$ & 30 & 700 & 1 & $\mathbb{N}$ & $\mathrm{N}$ & 15 & 100 & 15 \\
\hline WW215 & N & 20 & 700 & 1.5 & $\mathbf{N}$ & N & 15 & 70 & 15 \\
\hline WV216 & N & 20 & 700 & $<1$ & $N$ & $\mathbb{N}$ & 10 & 100 & 15 \\
\hline WW218 & N & 50 & 700 & 1 & $\mathrm{~N}$ & N & 20 & 150 & 30 \\
\hline WV219 & N & 50 & 700 & 1 & $\mathbf{N}$ & $N$ & $<10$ & 70 & 15 \\
\hline WV220 & N & 50 & 1,000 & 1 & $N$ & N & 20 & 100 & 20 \\
\hline WV221 & $\mathbb{N}$ & 30 & 1,000 & $<1$ & $\mathrm{~N}$ & N & 10 & 150 & 20 \\
\hline WV222 & $\mathbb{N}$ & 20 & 300 & 1 & $\mathbf{N}$ & N & N & 30 & 20 \\
\hline WV223 & $N$ & 30 & 700 & $<1$ & $\mathbf{N}$ & $N$ & 15 & 200 & 15 \\
\hline WV224 & $N$ & 30 & 700 & $<1$ & $N$ & $\mathrm{~N}$ & $<10$ & 70 & 10 \\
\hline WV225 & $N$ & 50 & 500 & 1 & $N$ & $N$ & 15 & 150 & 20 \\
\hline WV226 & $N$ & 50 & 1,000 & $<1$ & $\mathbf{N}$ & $N$ & 15 & 150 & 15 \\
\hline WV227 & N & 30 & 700 & $<1$ & $\mathrm{~N}$ & $\mathrm{~N}$ & 15 & 150 & 20 \\
\hline WV228 & $N$ & 50 & 700 & $<1$ & $N$ & $\mathbf{N}$ & $<10$ & 100 & 10 \\
\hline WV229 & $N$ & 30 & 700 & 1 & $\mathrm{~N}$ & $\mathrm{~N}$ & $<10$ & 70 & 10 \\
\hline WV230 & $N$ & 30 & 1,000 & $<1$ & $\mathbb{N}$ & $\mathbb{N}$ & 10 & 100 & 15 \\
\hline WV231 & $N$ & 50 & 700 & 1 & $N$ & $\mathbb{N}$ & $<10$ & 70 & 7 \\
\hline WV232 & $N$ & 50 & 1,000 & 1 & $\mathbf{N}$ & $\mathbf{N}$ & 15 & 100 & 20 \\
\hline WN233 & $\mathbb{N}$ & 30 & 1,000 & 1 & $N$ & $\mathrm{~N}$ & 30 & 150 & 15 \\
\hline WV234 & $N$ & 15 & 700 & 1 & $\mathbf{N}$ & N & 10 & 100 & 20 \\
\hline WV235 & N & 30 & 1,000 & $<1$ & $\mathbf{N}$ & N & 10 & 100 & 30 \\
\hline WV236 & N & 30 & 1,000 & 1 & $\mathbf{N}$ & $\mathbb{N}$ & 10 & 100 & 15 \\
\hline WN237 & N & 20 & 700 & $<1$ & $\mathbb{N}$ & $\mathbb{N}$ & 10 & 100 & 15 \\
\hline WV238 & N & 30 & 1,000 & 1 & N & N & 20 & 100 & 20 \\
\hline WV239 & N & 20 & 1,000 & 1 & $\mathbf{N}$ & N & 15 & 100 & 20 \\
\hline WV240 & N & 30 & 700 & 1 & N & N & 10 & 70 & 15 \\
\hline WV241 & N & 30 & 500 & 1 & $\mathbf{N}$ & N & 10 & 70 & 20 \\
\hline WV242 & $\mathrm{N}$ & 20 & 500 & 1 & $N$ & N & $<10$ & 50 & 10 \\
\hline WV243 & $N$ & 30 & 500 & 1 & $N$ & $\mathrm{~N}$ & $<10$ & 50 & 150 \\
\hline WV244 & N & 30 & 700 & 1 & $\mathrm{~N}$ & $N$ & 30 & 100 & 700 \\
\hline WV245 & $\mathbf{N}$ & 30 & 700 & 1 & N & $N$ & 15 & 70 & 700 \\
\hline WV246 & $N$ & 50 & 1,000 & $<1$ & $N$ & $N$ & 10 & 100 & 150 \\
\hline WV247 & $\mathbb{N}$ & 30 & 500 & 1 & $N$ & $\mathrm{~N}$ & 10 & 70 & 200 \\
\hline WV248 & $\mathrm{N}$ & 30 & 700 & $<1$ & N & $\mathrm{N}$ & 20 & 150 & 150 \\
\hline WV249 & $\mathrm{N}$ & 50 & 700 & 1 & $N$ & $\mathrm{~N}$ & 10 & 70 & 20 \\
\hline WV261 & $N$ & 30 & 700 & 1 & N & N & 30 & 100 & 150 \\
\hline WV268 & N & 30 & 700 & 1 & $\mathrm{~N}$ & 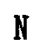 & 20 & 70 & 30 \\
\hline
\end{tabular}


Table 2. Gold and spectrographic analyses of 238 B-horizon soil samples.--Continued

Sample Ga ppm-s Ge ppm-s La ppm-s in ppm-s Mo ppm-s Nb ppm-s Ni ppm-s Pb ppm-s Sb ppm-s Sc ppm-s

\begin{tabular}{|c|c|c|c|c|c|c|c|c|c|c|}
\hline WV213 & 50 & N & $<50$ & 1,000 & N & $<20$ & 20 & 50 & N & 7 \\
\hline WV214 & 30 & $\mathbb{N}$ & $<50$ & 500 & N & $<20$ & 30 & 30 & $\mathbf{N}$ & 7 \\
\hline WV215 & 30 & $\mathbb{N}$ & $<50$ & 1,000 & $\mathbb{N}$ & $\mathrm{N}$ & 20 & 30 & $\mathrm{~N}$ & 7 \\
\hline WV216 & 30 & $\mathbf{N}$ & $<50$ & 500 & $\mathbb{N}$ & $<20$ & 30 & 30 & $\mathbb{N}$ & 7 \\
\hline WV218 & 30 & $\mathbb{N}$ & $<50$ & 3,000 & N & $<20$ & 30 & 50 & $\mathbb{N}$ & 10 \\
\hline WW219 & 30 & N & $<50$ & 700 & $<5$ & $<20$ & 15 & 30 & $\mathrm{~N}$ & 7 \\
\hline WV220 & 50 & N & $<50$ & 700 & $N$ & $<20$ & 30 & 50 & $\mathrm{~N}$ & 10 \\
\hline WV221 & 50 & $\mathrm{~N}$ & 50 & 300 & N & 20 & 30 & 70 & $\mathbb{N}$ & 7 \\
\hline W2222 & 7 & $\mathrm{~N}$ & $<50$ & 500 & $N$ & N & $<5$ & 10 & $\mathbb{N}$ & $<5$ \\
\hline WV223 & 50 & N & $<50$ & 700 & $N$ & $<20$ & 50 & 20 & $N$ & 7 \\
\hline WV224 & 30 & N & $<50$ & 700 & $N$ & $<20$ & 15 & 30 & N & 7 \\
\hline WV225 & 30 & $\mathbb{N}$ & $<50$ & 300 & N & $<20$ & 30 & 30 & $\mathbf{N}$ & 7 \\
\hline WV226 & 50 & $\mathrm{~N}$ & 50 & 500 & $\mathrm{~N}$ & $<20$ & 30 & 50 & $\mathbf{N}$ & 10 \\
\hline WV227 & 50 & N & 50 & 1,000 & $\mathrm{~N}$ & $<20$ & 15 & 50 & $\mathrm{~N}$ & 7 \\
\hline WV228 & 50 & N & 70 & 300 & 7 & $<20$ & 20 & 50 & N & 7 \\
\hline WV229 & 30 & $\mathrm{~N}$ & 70 & 200 & $\mathrm{~N}$ & $<20$ & 7 & 50 & $\mathrm{~N}$ & 5 \\
\hline WV230 & 50 & $\mathrm{~N}$ & $<50$ & 300 & $\mathrm{~N}$ & $<20$ & 10 & 50 & N & 7 \\
\hline WV231 & 30 & $\mathrm{~N}$ & 70 & 300 & $\mathrm{~N}$ & $<20$ & 7 & 70 & $\mathrm{~N}$ & 5 \\
\hline WV232 & 50 & N & 50 & 500 & N & 20 & 20 & 50 & N & 7 \\
\hline WV233 & 50 & N & 50 & 1,500 & N & 20 & 30 & 50 & $\mathbf{N}$ & 7 \\
\hline WV234 & 30 & N & $<50$ & 300 & N & $<20$ & 30 & 20 & $\mathrm{~N}$ & 7 \\
\hline WV235 & 30 & $\mathrm{~N}$ & $<50$ & 300 & N & $<20$ & 15 & 70 & $\mathrm{~N}$ & 7 \\
\hline WV236 & 30 & $\mathrm{~N}$ & $<50$ & 500 & $\mathrm{~N}$ & $<20$ & 15 & 50 & N & 7 \\
\hline WV237 & 30 & $\mathrm{~N}$ & $<50$ & 500 & $\mathrm{~N}$ & $<20$ & 30 & 20 & $\mathrm{~N}$ & 7 \\
\hline WV238 & 30 & N & $<50$ & 1,500 & $\mathrm{~N}$ & $<20$ & 50 & 30 & $\mathrm{~N}$ & 7 \\
\hline WV239 & 50 & N & $<50$ & 1,000 & $N$ & $<20$ & 20 & 50 & $\mathrm{~N}$ & 7 \\
\hline WV240 & 30 & N & $<50$ & 700 & $N$ & $<20$ & 15 & 30 & $\mathrm{~N}$ & 7 \\
\hline WV241 & 30 & $\mathrm{~N}$ & $<50$ & 150 & $N$ & $<20$ & 20 & 30 & N & 7 \\
\hline WV242 & 20 & N & $<50$ & 500 & $N$ & $<20$ & 15 & 30 & $\mathrm{~N}$ & 5 \\
\hline WV243 & 30 & N & $<50$ & 150 & $<5$ & $<20$ & 10 & 30 & $\mathrm{~N}$ & 5 \\
\hline WV244 & 30 & N & $<50$ & 500 & $<5$ & $<20$ & 50 & 30 & N & 7 \\
\hline WV245 & 30 & $\mathrm{~N}$ & 50 & 1,500 & 5 & $<20$ & 15 & 30 & $\mathrm{~N}$ & 5 \\
\hline WV246 & 50 & N & $<50$ & 700 & 5 & 20 & 20 & 50 & $\mathrm{~N}$ & 7 \\
\hline WV247 & 30 & $\mathrm{~N}$ & $<50$ & 500 & 5 & $<20$ & 15 & 70 & N & 5 \\
\hline WV248 & 30 & N & 50 & 1,000 & $\mathrm{~N}$ & $<20$ & 30 & 30 & $\mathrm{~N}$ & 7 \\
\hline WV249 & 30 & N & 50 & 1,000 & $\mathrm{~N}$ & $<20$ & 15 & 30 & N & 7 \\
\hline WV261 & 30 & $\mathrm{~N}$ & 70 & 700 & $N$ & $<20$ & 70 & 70 & $N$ & 7 \\
\hline WV268 & 30 & $\mathrm{~N}$ & 50 & 1,500 & $<5$ & $<20$ & 20 & 50 & $\mathrm{~N}$ & 5 \\
\hline
\end{tabular}


Table 2. Gold and spectrographic analyses of 238 B-horizon soil samples.--Continued

\begin{tabular}{|c|c|c|c|c|c|c|c|c|c|}
\hline Sample & Sn ppm-s & Sr ppm-s & Th ppm-s & V ppm-s & W ppm-s & $Y \quad p p m-s$ & Zn ppm-s & Zr ppm-s & Au $p p m-a$ \\
\hline WV213 & N & 500 & N & 100 & N & 20 & $<200$ & 300 & .001 \\
\hline WV214 & N & 500 & N & 100 & N & 15 & $<200$ & 150 & .002 \\
\hline WV215 & N & 300 & N & 70 & $\mathrm{~N}$ & 15 & $<200$ & 70 & .001 \\
\hline WV216 & N & 500 & N & 100 & N & 15 & N & 100 & .001 \\
\hline WW218 & $\mathrm{N}$ & 300 & N & 100 & N & 20 & $<200$ & 200 & .001 \\
\hline WV219 & N & 500 & N & 70 & $\mathrm{~N}$ & 15 & N & 300 & $<.001$ \\
\hline WV220 & N & 300 & N & 100 & N & 20 & N & 300 & .001 \\
\hline WV221 & N & 500 & N & 150 & N & 20 & N & 500 & .001 \\
\hline WV222 & N & 300 & N & 30 & N & $<10$ & 500 & 30 & .002 \\
\hline WV223 & $\mathrm{N}$ & 500 & N & 150 & N & 15 & N & 300 & .002 \\
\hline WV224 & N & 500 & N & 70 & N & 10 & N & 100 & .001 \\
\hline WV225 & N & 300 & N & 150 & N & 10 & $<200$ & 200 & .002 \\
\hline WV226 & $N$ & 700 & N & 150 & N & 20 & N & 500 & .005 \\
\hline WV227 & N & 700 & N & 150 & N & 15 & N & 70 & .001 \\
\hline WW228 & $\mathrm{N}$ & 500 & N & 100 & N & 15 & N & 300 & .001 \\
\hline WV229 & $\mathrm{N}$ & 500 & N & 70 & N & 15 & N & 200 & .001 \\
\hline WV230 & $\mathrm{N}$ & 300 & N & 100 & N & 15 & N & 300 & .002 \\
\hline W231 & N & 1,000 & N & 70 & N & 10 & N & 100 & .001 \\
\hline WV232 & $N$ & 300 & N & 100 & N & 15 & $<200$ & 300 & .002 \\
\hline WV233 & N & 500 & N & 100 & N & 15 & $<200$ & 500 & $<.001$ \\
\hline WV234 & N & 500 & N & 70 & N & 15 & N & 150 & .001 \\
\hline WV235 & N & 500 & N & 150 & N & 20 & N & 200 & .001 \\
\hline WV236 & N & 700 & $\mathrm{~N}$ & 100 & N & 15 & N & 200 & $<.001$ \\
\hline WV237 & N & 300 & N & 150 & N & 10 & N & 150 & $<.001$ \\
\hline WV238 & N & 300 & N & 100 & $\mathrm{~N}$ & 15 & 300 & 150 & .001 \\
\hline WV239 & N & 700 & N & 100 & N & 20 & 200 & 200 & .001 \\
\hline WV240 & N & 500 & N & 100 & N & 20 & 200 & 200 & $<.001$ \\
\hline WV241 & N & 300 & N & 100 & N & 15 & $<200$ & 200 & .001 \\
\hline WV242 & N & 300 & N & 70 & N & 10 & N & 70 & .001 \\
\hline WV243 & N & 150 & N & 70 & N & 15 & N & 150 & .002 \\
\hline WV244 & N & 300 & N & 150 & N & 20 & N & 200 & .012 \\
\hline WV245 & N & 300 & N & 100 & N & 15 & $<200$ & 150 & .044 \\
\hline WV246 & N & 300 & N & 150 & N & 30 & $\mathbf{N}$ & 500 & .002 \\
\hline WV247 & N & 300 & N & 100 & N & 15 & N & 100 & .016 \\
\hline WV248 & N & 700 & N & 150 & N & 20 & N & 200 & .038 \\
\hline WV249 & N & 300 & N & 100 & N & 20 & $<200$ & 150 & .005 \\
\hline WV261 & $\mathrm{N}$ & 300 & N & 150 & N & 20 & $<200$ & 200 & .001 \\
\hline WV268 & N & 200 & N & 100 & N & 15 & $<200$ & 200 & .013 \\
\hline
\end{tabular}

